\title{
Finite irreducible linear 2-groups of degree 4
}

\author{
Dane Laurence Flannery
}

September 1992

A thesis submitted for the degree of Doctor of Philosophy of the Australian National University 
Declaration

The work in this thesis is my own, unless otherwise stated.

Dhament

D. L. Flannery 


\section{Acknowledgements}

I acknowledge with deep gratitude the assistance given to me at all stages in the preparation of this thesis by my supervisor, Dr L. G. Kovács. In particular, I am grateful for the help he gave me in improving the exposition in Chapter 5.

I am indebted to my advisor, Dr E. A. O'Brien, for many invaluable discussions, and for proof-reading drafts of this thesis.

Thanks are due also to Dr M. F. Newman and the students and members of the MRS for providing a congenial place in which to work (at least when I was corporeally there).

Finally, I thank ANU and the Australian Government for financial assistance in the form of an APRA scholarship. 


\section{Abstract}

This thesis is concerned primarily with listing the finite irreducible 2-subgroups of $G L(4, \mathrm{C})$.

The problem falls naturally into three separate cases. We present a solution in the most difficult case, thereby establishing the methods that may be applied to the others.

There are three major steps in this solution. The first is part of a solution to a more general problem, namely that of describing the poset of finite submodules of the direct sum of two modules. A well-known theorem of Remak gives a canonical description of such submodules; we give a complementary theorem that describes certain inclusion relations between the submodules. Next we solve a group extension problem, by adapting results of Huebschmann to determine the orders of certain second cohomology groups. Finally, we solve a conjugacy problem, using various $a d$ hoc techniques and the theory developed in the first step.

These methods produce a complete and irredundant list of $G L(4, \mathbb{C})$-conjugacy class representatives of the groups in the first of the three cases. 


\section{Contents}

Abstract

List of Figures vi

1 Introduction 1

1.1 Discussion of the main problem .............. 1

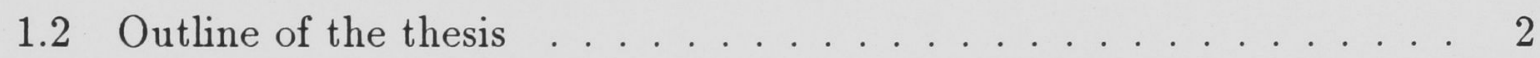

1.3 Notation and conventions ................. 4

2 Preliminaries 6

2.1 Some ring and module theory ... . . . . . . . . . . 6

2.2 Abelian transitive permutation groups . . . . . . . . . . 10

2.3 Irreducibility . . . . . . . . . . . . . . . 11

2.4 Isomorphisms for irreducible linear groups . . . . . . . . . . . 14

3 Cohomology of group extensions $\quad 16$

3.1 Cohomology theory of finite groups . . . . . . . . . . . 16

3.2 The LHS spectral sequence . . . . . . . . . . . . . . 26

3.3 Differentials in the LHS spectral sequence . . . . . . . . . . . 31

3.4 Cohomology of dihedral groups ................. 34

4 Submodule lattices of direct sums $\quad 44$

4.1 Background and basic definitions .............. 44

4.2 The submodules of $U \oplus V \ldots \ldots \ldots$. . . . . . . . . . 47

4.3 The Hasse diagram of $\mathcal{L}(U \oplus V) \ldots \ldots \ldots$

4.4 Examples ........................ 60 
5 The case $T=V_{4} \quad 66$

5.1 Submodule properties of $A_{A} \ldots \ldots \ldots 66$

5.2 Finite $V_{4}$-submodules of $B \ldots \ldots \ldots$

5.3 The extension problem ...................... 82

5.4 The conjugacy problem ........................... 91

$\begin{array}{lr}\text { References } & 107\end{array}$

$\begin{array}{lr}\text { Index } & 109\end{array}$ 


\section{List of Figures}

3.1 LHS spectral sequence differentials of total degree at most $2 \ldots 30$

4.1 Drawing the Hasse diagram of a Cartesian product . . . . . . 47

4.2 Illustration of Theorem $4.2 .1 \ldots \ldots \ldots \ldots$. . . . . . . . 49

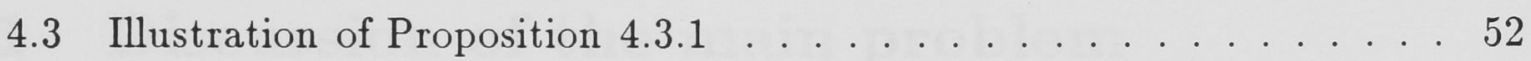

4.4 Illustration of Proposition $4.3 .3 \ldots \ldots \ldots \ldots \ldots$

4.5 Some inclusion relations in the interval $\mathcal{L}\left(U_{1} \oplus V_{1} / X_{2} \oplus Y_{2}\right) \ldots \ldots 56$

4.6 The atlas of the Hasse diagram of $\mathcal{L}(\operatorname{rad} A / \operatorname{soc} A)$ for $p=2 \ldots \ldots 6$

4.7 The Cartesian product page in the atlas of $\mathcal{H} \ldots \ldots \ldots 63$

4.8 The Hasse diagram of the subgroup lattice of $C_{4} \times C_{4} \ldots \ldots \ldots 6$

4.9 The Hasse diagram of $\mathcal{L}(3,2) \ldots \ldots \ldots \ldots \ldots$

5.1 The Hasse diagram of $\mathcal{L}(F(i+1, j+1,1,1,0,0) / F(i, j, 0,0,0,1)) \quad \ldots 79$

5.2 The Hasse diagram of $\mathcal{L}(F(i+1, j+1,1,1,1,1) / F(i, j, 0,0,1,1)) \quad \ldots 79$ 


\section{Chapter 1}

\section{Introduction}

\subsection{Discussion of the main problem}

For each natural number $n$, denote by $G L(n)$ the group of invertible $n \times n$ matrices with entries in the field of complex numbers $\mathbb{C}$. By a linear group of degree $n$ we mean a subgroup of $G L(n)$. The natural equivalence relation for linear groups is linear isomorphism - that is, conjugacy in the relevant $G L(n)$. Accordingly, each class of linear groups considered here will be a union of conjugacy classes, and a "list" of a class of linear groups means a list of conjugacy class representatives of the groups. It is desirable to provide with such a list an algorithm which, given a suitably described linear group, returns the representative of its conjugacy class.

The starting point of this thesis is the paper of Conlon (1976), in which such a list of finite irreducible linear $p$-groups of degree $p$, for each prime $p$, is provided. It is natural to try to do the same for irreducible $p$-groups of degree $p^{2}$. For general $p$ this is too difficult, and so in this thesis we deal only with irreducible 2 -groups of degree 4 .

The list constructed in this thesis, like Conlon's, is infinite. In both cases, the conjugacy class representatives which appear are sorted into a finite number of families. The members of any one family admit a common description in terms of integer parameters: each member of a family is labelled by a unique parameter string. This description amounts to giving a generating set of (monomial) matrices for each group. The entries of these matrices are explicit functions of the parameters.

The motivation for this thesis arises from a current aim in computational group theory to develop better "soluble quotient algorithms". These algorithms accept 
as input a group description (for example, a finite presentation) and produce as output a list of all sufficiently small finite soluble quotients of the group with that description. Some such algorithms envisaged will make use of databases containing all "sufficiently small" finite soluble groups which are primitive in the sense that they admit faithful primitive permutation representations. Listing groups of this kind is equivalent to listing irreducible soluble subgroups of $G L(n, p)$, the general linear group of degree $n$ over the field of order $p$. Irreducible soluble subgroups of $G L(n, p)$ are very closely related (for example, abstractly isomorphic) to subgroups of $G L(n)$. This close relationship provides the practical motivation for the thesis as a preparatory step towards extending the presently available databases. The next step in that direction is to obtain lists of irreducible 2-subgroups of $G L(4, p)$ for each odd prime $p$.

Most of the results in this thesis apply directly also to linear groups over any field that is algebraically closed (or, more weakly, over any field containing all 2-power roots of unity) with characteristic not 2 . We work over $\mathbb{C}$ only for convenience.

\subsection{Outline of the thesis}

This thesis is a report on a solution to the major part of the listing problem outlined in the previous section. The material is organised into five chapters. Chapter 2 is devoted to setting out some background theory, with specific reference to the listing problem. In order to describe the organisation of material in subsequent chapters, we will now explain the basic features of the problem a little further.

Denote by $M(4)$ and $D(4)$ the groups of monomial matrices and diagonal matrices in $G L(4)$; recall that $D(4)$ is normal in $M(4)$. We consider elements of the natural 4-dimensional space $\mathbb{C}_{4}$ as column vectors, and $G L(4)$ acts on the left. The group of permutation matrices in $G L(4)$ will be denoted $S_{4}$. Clearly, $M(4)$ is the semidirect product of $D(4)$ and $S_{4}$. Indeed, it is well-known that $M(4)$ is the wreath product $\mathbb{C}^{\times}$wr $S_{4}$, where $\mathbb{C}^{\times}$denotes the multiplicative group of $\mathbb{C}$. The base group of this wreath product is $D(4)$ and its top group is $S_{4}$. In fact, $S_{4}$ permutes the nonzero entries of each element of $D(4)$, numbered 1 to 4 in order starting at the first row. Also note that $S_{4}$ acts (via matrix multiplication) as the symmetric group on the set of orthonormal basis vectors $e_{i}(1 \leq i \leq 4)$, where $e_{i}$ has 1 in the $i$ th position and zeroes elsewhere. 
Each finite 2-subgroup of $G L(4)$, being nilpotent, is monomial (see Dixon (1971), p.76, Theorem 4.6B), by which we mean that it is conjugate to a subgroup of $M(4)$. There are two important groups associated to a subgroup $G$ of $M(4)$. The first of these is the diagonal subgroup $D(4) \cap G$ of $G$. The second is the projection group $D(4) G \cap S_{4}$. This is the image of the restriction to $G$ of the canonical projection map of $M(4)$ onto $S_{4}$. The following proposition states that the projection group of an irreducible $G$ must be transitive.

Proposition 1.2.1 Let $B$ denote the subgroup of $D(4)$ of all elements of 2-power order. A finite 2 -subgroup $G$ of $G L(4)$ is conjugate to a subgroup of $B S_{4}$. Furthermore, if $G \leq B S_{4}$ and $G$ is irreducible then $B G=B T$ for some transitive 2 -subgroup $T$ of $S_{4}$.

Proof. The first assertion is clear from the discussion above. The projection map of $G$ into $S_{4}$ has kernel $B \cap G$, and so $G / B \cap G \cong S_{4} \cap B G=T$. If $G$ is irreducible and $T$ intransitive, then a proper subset of the $e_{i}$ defined above is invariant under $T$ and hence also under $B T$, and a proper non-zero subspace of $\mathbb{C}^{4}$ admits $G$.

A transitive 2 -subgroup of $S_{4}$ has order 4 or 8 . There are 3 cyclic subgroups of $S_{4}$ of order 4 and each is transitive. These form a single conjugacy class of subgroups of $S_{4}$. A subgroup of order 8 of $S_{4}$ is a Sylow 2-subgroup and so is isomorphic to $C_{2}$ wr $C_{2} \cong D_{8}$. Each of the three Sylow 2-subgroups of $S_{4}$ contains a (unique) cyclic subgroup of order 4 and hence is transitive. Of course, the Sylow 2-subgroups of $S_{4}$ form a single conjugacy class of subgroups of $S_{4}$. The only fours group in $S_{4}$ that is transitive is $V_{4}=\langle(12)(34),(13)(24)\rangle$. This is normal in $S_{4}$ and hence is contained in every $D_{8}$ in $S_{4}$. We choose a fixed $C_{4}$ and $D_{8}$ in $S_{4}$ and denote these by $C$ and $D$ respectively, assuming that $C<D$. Thus, a finite irreducible 2-subgroup of $B S_{4}$ is conjugate (by a permutation matrix) to one whose projection group is contained in $D$. By Proposition 1.2.1 and the preceding discussion, the problem has been reduced to the following.

For each case $T=V_{4}, C$ and $D$, find all finite irreducible 2-subgroups $G$ of $B S_{4}$ such that $B G=B T$.

Next, we indicate our approach to a solution of this reduced problem.

Note that $B$ is certainly a module for each $T$. For $G$ as in the above statement, $B \cap G$ is a finite $T$-submodule of $B$ and $G$ is an extension of $B \cap G$ by $T$. Therefore, 
the first major task we undertake is to determine all the possibilities for $B \cap G$ by determining all the finite $T$-submodules of $B$. (This listing problem is the source of much of the complexity of the full listing problem.) It is useful also to have information about the inclusion relations between these submodules. For instance, such information will assist in translating the relevant part of the full list for $\mathbb{C}$ into that for finite prime fields. In other words, we are really interested in the poset of finite $T$-submodules of $B$. For $T \neq D$, it will be seen that (apart from a minor perturbation involving certain submodules of order 4), this poset is the union of countably many finite posets. Each of these is the lattice of submodules of the direct sum of two modules, an object studied in full generality in Chapter 4 .

Of course, $B \cap G$ and $T$ do not in general determine $G$. It is necessary to solve an "extension problem"; namely, given a transitive 2-subgroup $T$ of $S_{4}$ and a finite $T$-submodule $U$ of $B$, we need to determine all finite 2 -subgroups $G$ of $B T$ such that $B \cap G=U$ and $B G=B T$. Such $G$ need not be irreducible, and therefore we will need to be able to identify the reducible $G$. The solution presented here to this extension problem involves spectral sequences and cohomology theory of finite groups. The relevant results in this area are given in Chapter 3. We use these results to construct a list of $B T$-conjugacy classes of the relevant $G$. However, non-conjugate subgroups of $B T$ may still be conjugate in $G L(4)$. The final stage of the problem is to determine the remaining conjugacies.

These procedures are carried out in Chapter 5 for $T=V_{4}$. The results in this and other chapters are developed in a general context which allows them to be applied routinely to the cases $T=C$ and $T=D$. The calculations in these cases are significantly easier than those for $T=V_{4}$ and are not included here. At the time of writing, all that remains to be done to obtain a full list of finite irreducible 2 -subgroups of $G L(4)$ is to complete the solution of the conjugacy problem for $T=D$. The full list will appear elsewhere, as part of on-going work towards the overall objective of listing irreducible 2-subgroups of $G L(4, p)$.

\subsection{Notation and conventions}

Group theory notation is standard and mostly follows Robinson (1982). The centre of a group $G$ is denoted $\zeta(G)$ and its Frattini subgroup $\Phi(G)$. The commutator of two elements $g$ and $h$ of $G$ is $[g, h]=g^{-1} h^{-1} g h$. If $G$ is abelian, its rank is denoted 
$\operatorname{rk}(G)$. If $G$ is an abelian $p$-group, $\Omega_{k}(G)$ denotes the subgroup of all elements of order at most $p^{k}$.

The kernel of a map $f$ is abbreviated as $\operatorname{ker} f$ and its image as im $f$. Most maps will be written on the right. Characters will be written on the left, as is the tradition. To retain coherence, representations also will be written on the left. In ambiguous situations (such as homomorphisms between linear groups - it is unclear whether such maps should be considered abstractly and act on the right, or as representations and act on the left) the choice will be determined by, and be clear from, the context. Actions are usually right actions, although there are exceptions (such as modules for linear groups).

If $M$ is a finite module, we speak of the rank and exponent of the finite abelian group $M$. If $\mathcal{S}$ is a subset of $M,\langle\mathcal{S}\rangle$ denotes the subgroup generated by $\mathcal{S}$.

We write an element of $D(4)$ as the 4-tuple consisting of its non-zero entries, starting at the $(1,1)$ th position. We use cycle notation to denote elements of $S_{4}$. This should cause no confusion. For example, (12) will denote both a permutation of the four point set, and the element of $G L(4)$ that results from interchanging the first and second rows of the identity matrix. The context will indicate which one of these is implied. Premultiplication of $m \in G L(4)$ by $s \in S_{4}$ permutes the rows of $m$ (numbered in order from first to last) as $s$ permutes the four point set; postmultiplication permutes the columns in the same fashion.

The Galois field of order $q$ is denoted $F_{q}$, and the vector space of dimension $n$ over $\mathrm{F}_{q}$ is denoted $V(n, q)$.

When we speak of an irreducible character of a group, we mean an irreducible complex character. If two representations of a finite linear group afford the same character, then these representations are equivalent. Also, any finite abelian linear group is diagonalisable.

Finally, we fix the following notation introduced in Section 1.2:

- $B$ is the 2-torsion subgroup of $D(4)$,

- $V_{4}$ is the unique normal subgroup of $S_{4}$ of order 4 ,

- $D$ is the dihedral group $\left\langle V_{4},(1234)\right\rangle$ of order 8 in $S_{4}$,

- $C$ is the unique cyclic subgroup $\langle(1234)\rangle$ of order 4 in $D$.

An index of notation and terms is given at the end of the thesis. 


\section{Chapter 2}

\section{Preliminaries}

In this chapter we present some preliminary results. We will begin by showing how the problem of listing finite submodules of $B$ may be formulated equivalently in a group algebra context. Then we give some elementary facts about $C$ and $V_{4}$ as abstract permutation groups. Finally, we discuss irreducibility of and isomorphism between finite linear groups.

\subsection{Some ring and module theory}

The first step in the programme is the listing of the finite $T$-submodules of $B$, for each case $T=V_{4}, C$ and $D$. In this step, it suffices to assume that $T=V_{4}$ or $C$, since the set of $D$-submodules of $B$ is the intersection of the sets of $V_{4}$-submodules and $C$-submodules of $B$. So for this section, $T$ is regular in its action on the entries of an element of $B$.

As we mentioned in Section 1.2, the poset of finite $T$-submodules of $B$ is the union of certain finite posets. In turn, a submodule in one of these posets may be regarded as a submodule of the regular module of a certain group algebra. This is essentially the context in which we undertake the listing of the finite $T$-submodules of $B$. We now discuss this context a little further. Since $T$ is regular, each element of $T$ is determined by its action on a fixed element of the four point set $\{1, \ldots, 4\}$. So we denote the elements of $T$ as $t_{i}(1 \leq i \leq 4)$, where $t_{i}$ moves the point 1 to the point $i$. Thus $T$ in its right regular permutation representation permutes the $t_{i}$ as it permutes the elements of the four point set: $t_{i} t=t_{i t}$ for each $t \in T$. Suppose that $U$ is a finite $V_{4}$-submodule of $B$ whose exponent is $2^{n}$ and denote 
by $A$ the group algebra $\left(\mathbb{Z} / 2^{n} \mathbb{Z}\right) T$. Choose a primitive $2^{n}$ th root of unity $\omega$. For each $\left(\lambda_{1}, \lambda_{2}, \lambda_{3}, \lambda_{4}\right) \in U$, we may write $\lambda_{i}=\omega^{\mu_{i}}$ for $1 \leq \mu_{i} \leq 2^{n}$. Define the map $f_{\omega}: U \rightarrow A$ by

$$
f_{\omega}:\left(\lambda_{1}, \lambda_{2}, \lambda_{3}, \lambda_{4}\right) \mapsto \sum_{i=1}^{4} \mu_{i} t_{i}
$$

By our definition of the $t_{i}$, for fixed $\omega$ the map $f_{\omega}$ is a $T$-isomorphism of $U$ onto a submodule of the right regular module $A_{A}$. Hence $f_{\omega}$ sets up a one-to-one correspondence between the set of finite $T$-submodules of $B$ of exponent at most $2^{n}$ and the set of submodules of $A_{A}$, which maps such a submodule of $B$ to its image in $A_{A}$ under $f_{\omega}$. If $\omega^{\prime}$ is another $2^{n}$ th root of unity then write $\omega^{\prime}=\omega^{r}$ for some odd $r$. Clearly, $f_{\omega}=r f_{\omega^{\prime}}$, so that $U f_{\omega}=U f_{\omega^{\prime}}$ and $f_{\omega^{\prime}}$ induces the same one-toone correspondence as $f_{\omega}$. This shows that listing the finite $T$-submodules of $B$ is equivalent to listing the submodules of $A_{A}$ for each positive integer $n$. In the rest of this section we record some properties of $A$ and $A_{A}$.

For the following discussion, let $R$ be a ring with 1 and $M$ a right $R$-module. The radical of $M, \operatorname{rad} M$, is the intersection of all the maximal submodules of $M$. (All the nonzero modules with which we deal in the applications are finitely generated, and hence have maximals.) The Jacobson radical of $R$ is the radical of $R_{R}$, and is denoted $\operatorname{rad} R$. This is a (two-sided) ideal of $R$. We say $R$ is a local ring if $R_{R}$ has a unique maximal submodule, which is of course $\operatorname{rad} R$.

Proposition 2.1.1 For a local ring $R$ and an $R$-module $M, \operatorname{rad} M=(\operatorname{rad} R) M$.

Proof. See Curtis \& Reiner (1981), p.116, 5.29.

Proposition 2.1.2 Let $R$ be a commutative local ring such that $R / \operatorname{rad} R$ is a field of characteristic $p$, and $H$ a finite $p$-group. Then $R H / \operatorname{rad} R H \cong R / \operatorname{rad} R$, so that $R H$ is a local ring and there is a unique isomorphism type of $R H$-module.

Proof. See Curtis \& Reiner (1981), p.115, 5.25. A simple module is monogenic (that is, generated by a single element), and hence is a homomorphic image of $R H_{R H}$. Since $R H_{R H}$ has a unique maximal submodule here, the third statement follows.

The hypotheses of Proposition 2.1.2 are certainly satisfied with $R=\mathbb{Z} / p^{n} \mathbb{Z}$. In this case, every simple $R H$-module has order $p$ by Proposition 2.1.2.

The ring $R$ is said to be self-injective (or quasi-Frobenius) if $R_{R}$ is an injective $R$-module. Every proper homomorphic image of $\mathbb{Z}$ is self-injective. In particular, $\mathbb{Z} / p^{n} \mathbb{Z}$ is self-injective. 
Proposition 2.1.3 Let $R$ be a self-injective commutative local ring and $H$ be $a$ finite abelian group. Then two submodules of $R H_{R H}$ are isomorphic if and only if they are equal.

Proof. The hypotheses imply that $R H$ is a self-injective ring [see Curtis \& Reiner (1962), p.402, Ex.2(d)]. Consequently, any homomorphism between submodules of $R H_{R H}$ may be extended to an endomorphism of $R H_{R H}$. An endomorphism of $R H_{R H}$ is multiplication on the left by an element of $R H$. This implies the conclusion, since $R H$ is a commutative ring.

Mostly, we apply Proposition 2.1.3 to finite $T$-submodules of $B$. In this context, the conclusion of the proposition is that two finite $T$-submodules of $B$ are isomorphic if and only if they are equal.

If $R$ is an Artinian ring then every nonzero $R$-module $M$ has a simple submodule. The sum of all the simple submodules of $M$ is called the socle of $M$ and is denoted $\operatorname{soc} M$. By Propositions 2.1.2 and 2.1.3, $A_{A}$ has a unique simple submodule, $\operatorname{soc} A$, and a unique maximal submodule, $\operatorname{rad} A$. This means that each nonzero submodule of $A_{A}$ is contained in $\operatorname{rad} A$ and contains $\operatorname{soc} A$.

Now we discuss duality. If $U$ is any (right) $R$-module, the annihilator of $U$ in $R$ is

$$
\operatorname{ann}_{R} U=\{r \in R \mid U r=0\}
$$

This is an ideal of $R$. Let $\left\{U_{i} \mid 1 \leq i \leq n\right\}$ be a set of submodules of $M$. Recall the familiar properties of annihilators:

- $U_{i} \leq U_{j} \Rightarrow \operatorname{ann}_{R} U_{j} \leq \operatorname{ann}_{R} U_{i}$

- $\operatorname{ann}_{R} \sum_{i=1}^{n} U_{i}=\bigcap_{i=1}^{n} \operatorname{ann}_{R} U_{i}$

- $\sum_{i=1}^{n} \operatorname{ann}_{R} U_{i} \leq \operatorname{ann}_{R} \bigcap_{i=1}^{n} U_{i}$

- $\operatorname{ann}_{R} a n n_{R} a n n_{R} U=\operatorname{ann}_{R} U$.

If $R$ and $H$ are as in Proposition 2.1.3 then there exists a non-degenerate associative symmetric bilinear form $f: R H \times R H \rightarrow R$. For instance, define $f(x, y)$ to be the coefficient of 1 in $x y$ (see Curtis \& Reiner (1981), p.196, 9.2). If $U$ is a submodule of $R H_{R H}$, set

$$
U^{\perp}=\{y \in R H \mid f(x, y)=0 \text { for all } x \in U\}
$$


It is easy to verify that $U^{\perp}=\operatorname{ann}_{R H} U$.

Part (i) of the next result shows that the map $U \rightarrow U^{\perp}$ is an inclusion reversing bijection of the lattice of submodules of $R H_{R H}$ onto itself.

Proposition 2.1.4 Let $R$ and $H$ be as in Proposition 2.1.3 and also suppose that $R$ is finite, $R / \operatorname{rad} R$ has characteristic $p$, and $H$ is a $p$-group. If $U$ is a submodule of $R H_{R H}$ then

(i) $\left(U^{\perp}\right)^{\perp}=U$

(ii) $|U|\left|U^{\perp}\right|=|R H|$.

Furthermore, if $U$ is monogenic then

(iii) $R H_{R H} / U^{\perp} \cong U$.

Proof. For a proof of (i) - the so-called "double annihilator property" - see Anderson \& Fuller (1973), p.286, Ex.13. The proof uses the fact that $R H$ is quasiFrobenius. To verify (iii), note that if $U$ is generated by one of its elements $u$, then the homomorphism of $R H_{R H}$ onto $U$ that maps $x \in R H$ to $u x$ has kernel ann ${ }_{R H} U$. Then (ii) follows by induction on $|U|$, using (i) and (iii).

Remark 2.1.5 When $U$ is not monogenic, we need not have $R H_{R H} / U^{\perp} \cong U$. For example, if $R=\mathbb{Z} / p \mathbb{Z}, H=C_{p} \times C_{p}$ and $U=\operatorname{rad} R H$, then $U^{\perp}=\operatorname{soc} R H$. Now $\operatorname{rad} R H$ has a unique minimal submodule, but $R H_{R H} / \operatorname{soc} R H$ does not - see Lemma 5.1.1.

In the next proposition we give subgroup generators of $\operatorname{rad} R H$ and $\operatorname{soc} R H$, for the special cases of $R$ and $H$ in which we will be interested later on.

Proposition 2.1.6 For $n \geq 1$, set $R=\mathbb{Z} / p^{n} \mathbb{Z}$ and let $H$ be a finite $p$-group. Then

$$
\operatorname{rad} R H=\langle p, x-1 \mid x \in H, x \neq 1\rangle
$$

and

$$
\operatorname{soc} R H=\left\langle p^{n-1} \sum_{x \in H} x\right\rangle
$$

Proof. The first assertion, which says that $\operatorname{rad} R H$ is generated by the augmentation ideal and $p$, is proved in Curtis \& Reiner (1981), p.115, 5.25. The second assertion is clear since in this case $\operatorname{soc} R H$ is the unique simple submodule of $R H_{R H}$. 


\subsection{Abelian transitive permutation groups}

To simplify notation in this section, we write $C(P)$ and $N(P)$, for a subgroup $P$ of $S_{n}$, to denote respectively the centraliser and normaliser of $P$ in $S_{n}$. Likewise, if $P$ is a subset of an $S_{n}$-module, $C(P)$ and $N(P)$ denote respectively the pointwise and setwise stabiliser of $P$ in $S_{n}$.

We take it as well-known that if $T$ is an abelian transitive subgroup of $S_{n}$ then $T$ is regular, and that $C(T) \cong T$ for every regular $T$ in $S_{n}$. In particular, if $T$ is abelian and regular then $C(T)=T$.

Lemma 2.2.1 If $T$ is a regular subgroup of $S_{n}$ then $\operatorname{Aut}(T)$ is realised as a group whose elements are restrictions of inner automorphisms of $S_{n}$. Consequently, $N(T) / C(T) \cong \operatorname{Aut}(T)$.

Proof. Each $\theta \in \operatorname{Aut}(T)$ may be regarded as a permutation representation of $T$. In fact, this permutation representation is regular and so is equivalent to the inclusion $\iota$ of $T$ in $S_{n}$. In other words, $t \theta=\sigma^{-1} \cdot t \iota . \sigma$ for some $\sigma \in S_{n}$, that is, $t \theta=t^{\sigma}$. The conclusion follows.

Remark 2.2.2 If $T$ is transitive but not regular, the conclusion of Lemma 2.2.1 need not hold. For example, consider $T=D$ and $n=4$. If the conclusion held, then, since $N(D)=D$, we would have $\operatorname{Aut}(D)=\operatorname{Inn}(D)$. But this is false.

Remark 2.2.3 If $T$ is an abelian transitive subgroup of $S_{4}$ then $N(T)$ splits over $C(T)=T$. For $N\left(V_{4}\right)=S_{4}$ and $\langle(123),(12)\rangle \cong S_{3}$ is a complement of $V_{4}$ in $S_{4}$. Also, $N(C)=D$ and of course $D$ splits over $C$.

Lemma 2.2.4 Let $T$ be an abelian transitive subgroup of $S_{n}$ and suppose that $U$ is a $T$-submodule of some $S_{n}$-module. Then $C_{T}(U)=1$ if and only if $C_{N(T)}(U)=1$.

Proof. We have $C(T)=T$. Assume that $C_{T}(U)=1$ and choose $s \in C_{N(T)}(U)$. If $t \in T$ then $[s, t] \in T \cap C(U)=C_{T}(U)=1$. Therefore, $s \in C(U) \cap C(T)=1$.

A regular subgroup of $S_{n}$ is not 2 -transitive, but when $n=4$ we have the following related result.

Lemma 2.2.5 Let $T$ be a regular subgroup of $S_{4}$. If $i, j, k, l \in\{1,2,3,4\}$ are distinct, then either there is $t \in T$ such that $\left(k^{t}, l^{t}\right)=(i, j)$ or there is $t \in T$ such that $\left(k^{t}, l^{t}\right)=(j, i)$. 
Proof. Choose $t \in T$ such that $k^{t}=i$. Either $l^{t}=j$, in which case we are done, or $l^{t}=k$. In the latter case, necessarily $i^{t}=j$ and $\left(k^{t^{2}}, l^{t^{2}}\right)=(j, i)$.

\subsection{Irreducibility}

By Proposition 1.2.1, the projection group of a finite irreducible 2-subgroup of $B S_{4}$ is a transitive 2-subgroup of $S_{4}$. However, a finite 2-subgroup of $B S_{4}$ with transitive projection group need not be irreducible. A (simple) condition on the diagonal subgroup, rather than the projection group, is equivalent to irreducibility. This is given in Theorem 2.3.5 below.

Lemma 2.3.1 Let $G$ be a finite 2-subgroup of $G L(4)$. Then any one of the following conditions implies that $G$ is reducible:

(i) $|G| \leq 16$;

(ii) $G$ has an abelian maximal subgroup;

(iii) $G$ has an abelian normal subgroup $A$ of index 4 such that $A<C_{G}(A)$.

Proof. Let $\chi$ be an irreducible constituent of the trace map of $G$. Then by Isaacs (1976), p.28, 2.29, $\chi(1)^{2} \leq|G: \zeta(G)|$. If $|G| \leq 16$, then $\chi(1)^{2} \leq 8$ and so $\chi$ has degree less than 4 . If $H$ is an abelian maximal subgroup of $G$ then by Isaacs (1976), p.30, Ex.2.9(b), $\chi(1) \leq|G: H|=2$. Again $G$ is reducible and so (i) and (ii) are verified. For (iii), note that if $A<C_{G}(A)$ then either $C_{G}(A)=G$ or $\left|C_{G}(A): A\right|=2$. In the first case, $A \leq \zeta(G)$ and so $\chi(1) \leq 2$. In the second case, $C_{G}(A) / A=C_{G}(A) / \zeta\left(C_{G}(A)\right)$ is cyclic and hence $C_{G}(A)$ is an abelian maximal subgroup of $G$, proving (iii) by (ii).

In particular, each finite abelian 2 -subgroup of $G L(4)$ is reducible.

Definition 2.3.2 For each $i, 1 \leq i \leq 4$, define the linear character $\alpha_{i}$ of $U$ by

$$
\alpha_{i}: u \mapsto u_{i, i}
$$

where $u \in B \cap G$ and, as usual, $u_{i, j}$ denotes the $(i, j)$ th entry of the matrix $u$.

Recall that a module for a group is called faithful if the identity is the only group element fixing the module elementwise. 
Lemma 2.3.3 Let $T$ be a transitive 2-subgroup of $S_{4}$ and suppose that $U$ is a faithful finite $T$-submodule of $B$. Then $\alpha_{i}=\alpha_{j}$ if and only if $i=j$.

Proof. It suffices to consider the case that $|T|=4$, since each $D_{8}$ in $S_{4}$ contains a transitive 2-subgroup of $S_{4}$ of order 4 . In that case, $T$ is normalised by a $D_{8}$ in $S_{4}$ and $V_{4} \leq N_{S_{4}}(T)$. The lemma is proved if we can establish that $\alpha_{j} \neq \alpha_{1}$ for $j \neq 1$, since $T$ permutes transitively the diagonal entries of an element of $B$.

Suppose that $j \neq 1$ and $\alpha_{j}=\alpha_{1}$. Denote the elements of $\{2,3,4\} \backslash\{j\}$ by $k$ and $l$. The hypothesis implies that the first and $j$ th entries of every element of $U$ are the same but the $k$ th and $l$ th entries of some $u \in U$ are distinct. Otherwise, $(1 j)(k l) \in C_{S_{4}}(U) \cap V_{4} \leq C_{N_{S_{4}}(T)}(U)=1$ by Lemma 2.2.4. By Lemma 2.2.5 there is $t \in T$ such that at least one of the following occurs: $\left(k^{t}, l^{t}\right)=(1, j)$ or $\left(k^{t}, l^{t}\right)=(j, 1)$. In either case $\alpha_{1}\left(u^{t}\right) \neq \alpha_{j}\left(u^{t}\right)$, a contradiction.

Proposition 2.3.4 Let $G$ be a finite 2 -subgroup of $B S_{4}$ such that $B G=B T$ for some transitive 2 -subgroup $T$ of $S_{4}$. If $B \cap G$ is faithful as $T$-module, then $G$ is irreducible.

Proof. Since $T$ is transitive, the linear characters $\alpha_{i}$ of the normal subgroup $B \cap G$ of $B T$ are pairwise $B T$-conjugate. Since $B$ is abelian and $B T=B G$, these characters are in fact pairwise $G$-conjugate.

First assume that $|T|=4$. Let $\alpha$ be the trace map of $G$ and $\beta$ an irreducible constituent of $\alpha$. Now $\alpha_{B \cap G}=\sum_{i=1}^{4} \alpha_{i}$; thus, for some $k, \alpha_{k}$ is an irreducible constituent of $\beta_{B \cap G}$. But the $\alpha_{i}$ are pairwise $G$-conjugate and so by Clifford's Theorem [see Dornhoff (1971), p.72, Theorem 14.1 (6)], $\beta_{B \cap G}=e \sum_{i=1}^{4} \alpha_{i}=e \alpha_{B \cap G}$ for some integer $e \geq 1$. The degree of $\beta_{B \cap G}$ is at most 4 and so $e=1$. Therefore $\alpha=\beta$ is irreducible.

If $|T|=8$, there is a subgroup $H$ of $G$ such that $B \cap H=B \cap G, B H=B V_{\mathbf{4}}$ and $V_{4}$ acts faithfully on $B \cap G$. By the preceding paragraph, $H$ is irreducible and hence $G$ is irreducible.

The next theorem follows from Lemma 2.3.1 (iii) and Proposition 2.3.4.

Theorem 2.3.5 Let $G$ be a finite 2-subgroup of $B S_{4}$ such that $B G=B T$ for some transitive 2 -subgroup $T$ of $S_{4}$ of order 4 . Then $G$ is irreducible if and only if $B \cap G$ is faithful as $T$-module. 
Given a transitive subgroup $T$ of $S_{4}$ of order 4 and a $T$-submodule $U$ of $B$, Theorem 2.3.5 shows that either all $G$ with $B \cap G=U$ and $B G=B T$ are irreducible, or all these $G$ are reducible. In the programme outlined in Section 1.2, irreducibility testing can therefore be done before solving the relevant extension problem. However, Theorem 2.3.5 fails when $|T|=8$.

Irreducibility guarantees several important properties that are useful in solving the conjugacy problem. These are given in Propositions 2.3.6 and 2.3.7.

Proposition 2.3.6 Let $G$ be a finite irreducible 2 -subgroup of $G L(4)$ with an abelian normal subgroup $A$ of index 4 . Then there is $x \in G L(4)$ such that $G^{x} \leq B S_{4}$ and $A^{x}=B \cap G^{x}$.

Proof. First note that by Lemma 2.3.1(ii), $A$ is a maximal abelian normal subgroup of $G$. Also, $A$ is diagonalisable (this depends on the algebraic closure of $\mathbb{C}$ ) and so there is $y \in G L(4)$ such that $A^{y} \leq B \cap G^{y}$. By maximality of $A, A^{y}=B \cap G^{y}$. However, it is not necessarily true that $G^{y} \leq B S_{4}$. Assume by the preceding, then, that $A=B \cap G$; we will show that the required linear isomorphism exists.

Let $\alpha$ be the trace map of $G$ and let $\alpha_{i}$ be as defined in Definition 2.3.2. Since $\alpha$ is an irreducible character of $G$, by Frobenius reciprocity the multiplicity of $\alpha$ in the induced character $\alpha_{i}^{G}$ is equal to the multiplicity of $\alpha_{i}$ in $\alpha_{A}$, which is 1 . Then $|G: A|=4$ implies that $\alpha_{i}^{G}$ has degree 4 and so $\alpha=\alpha_{i}^{G}$ for all $i$. The induced character $\alpha_{i}$ is afforded by a representation $\phi$ of $G$ as monomial matrices and furthermore $\phi(A) \leq B$. Of course, $\alpha$ is afforded by the inclusion $\iota$ of $G$ in $G L(4)$. This means that $\phi$ is equivalent to $\iota$, and therefore $G^{x} \leq B S_{4}$ and $B \cap G^{x}=A^{x}$ for some $x \in G L(4)$.

Proposition 2.3.7 Let $G$ be a finite irreducible 2 -subgroup of $B S_{4}$. Then

$$
N_{G L(4)}(B \cap G) \leq M(4)
$$

Proof. Let $V$ be the natural $C$-space of $4 \times 1$ column vectors and denote by $V_{j}$ the subspace spanned by the vector $v_{j}$ with 1 in the $j$ th position and zeroes elsewhere. We may write $\left.V\right|_{B \cap G}=\oplus_{j=1}^{4} V_{j}$. Choose $x \in N_{G L(4)}(B \cap G)$. Then $x V_{j}$ is a $(B \cap G)$-module and so affords a 1-dimensional representation $\phi_{j}$ of $B \cap G$. That is, for each $g \in B \cap G$,

$$
\phi_{j}(g) x v_{j}=g x v_{j} .
$$


Whence for each $i, 1 \leq i \leq 4$,

$$
\phi_{j}(g) x_{i j}=\alpha_{i}(g) x_{i j}
$$

By Lemma 2.3.3, the $\alpha_{i}$ are distinct so that for fixed $j, x_{i j} \neq 0$ for unique $i$. In other words, each column of $x$ has a single nonzero entry. The invertibility of $x$ then implies that $x$ is monomial.

Remark 2.3.8 Suppose that $G$ is as in Proposition 2.3.7 and $G^{d} \leq B S_{4}$ for some $d \in D(4)$. If $t \in T=B G \cap S_{4}$, we have $[d, t] \in B S_{4} \cap D(4)=B$. Since $T$ is transitive, this means that each entry of $d$ may be written as a $C_{2 \infty}$-multiple of a fixed entry of $d$. Thus $d$ is a scalar multiple of an element of $B$.

\subsection{Isomorphisms for irreducible linear groups}

Consider the following isomorphism question for a finite irreducible subgroup $G$ of $G L(n)$ :

If a subgroup of $G L(n)$ is abstractly isomorphic to $G$, is it linearly isomorphic to $G$ ?

For $n=p$ and $G$ a finite $p$-group the answer is yes - see Conlon (1976), Proposition 4.2. This is proved by first listing the $G L(p)$-conjugacy class representatives of all possible $G$ and then verifying that no two groups in the list are isomorphic. A nonconstructive proof (for this and other $n$ ) is desirable, because it would a priori reduce the difficulty of the conjugacy problem. In this thesis, we obtain only partial answers to the isomorphism question for $n=4$ (see the comment after Theorem 5.4.15).

It is important to be able to decide if an isomorphism between finite irreducible 2-subgroups of $B S_{4}$ respects diagonal subgroups. This is because each group in all but a very few of the families in the final list of conjugacy class representatives has a unique abelian normal subgroup of the same index as its diagonal subgroup.

Theorem 2.4.1 Suppose that $G$ and $H$ are finite subgroups of $B S_{4}$ such that $B G=B H=B T$ for some transitive 2 -subgroup $T$ of $S_{4}$. If there is an isomorphism $\theta$ of $G$ onto $H$ such that $(B \cap G) \theta=B \cap H$, then there exists $s \in N_{S_{4}}(T)$ such that $(B \cap G)^{s}=B \cap H$. In particular, if $|T|=8$ then $B \cap G=B \cap H$. 
Proof. First suppose that $|T|=4$. Given $t \in T$, choose $g \in G$ such that $g B=t B$. Then choose $t^{*} \in T$ such that $g \theta B=t^{*} B$. Define a map $\tau: T \rightarrow T$ by $t \tau=t^{*}$. Now $\tau$ is well-defined. In fact, it may be verified readily that $\tau$ is an automorphism of $T$. Thus $\tau$ acts on $T$ as conjugation by an element $s$ say of $N_{S_{4}}(T)$, by Lemma 2.2.1. Let $x \in B \cap G$ and choose $d \in B$ such that $g \theta d=t \tau$. Then

$$
\begin{aligned}
(x \theta)^{s^{-1} t s} & =(x \theta)^{g \theta d} \\
& =\left(x^{g} \theta\right)^{d} \\
& =\left(x^{t} \theta\right)^{d} \\
& =x^{t} \theta
\end{aligned}
$$

since $B$ is abelian, so that

$$
\left(x^{t} \theta\right)^{s^{-1}}=(x \theta)^{s^{-1} t} .
$$

This shows that $\theta s^{-1}$ is a $T$-module isomorphism of $B \cap G$ onto $(B \cap H)^{s^{-1}}$ (the latter object is certainly a finite $T$-submodule of $B$ ). Hence, by Proposition 2.1.3, $B \cap G=(B \cap H)^{s^{-1}}$, verifying the claim for $|T|=4$.

Next take $T=D$ without loss of generality. There is a maximal subgroup $G_{1}$ of $G$ such that $B \cap G_{1}=B \cap G$ and $B G_{1}=B C$. Set $G_{2}=G_{1} \theta$. Then $B \cap G_{2} \leq B \cap H=\left(B \cap G_{1}\right) \theta \leq B \cap G_{2}$, so that $B \cap G_{2}=\left(B \cap G_{1}\right) \theta$. Since $G_{2} / B \cap G_{2} \cong C, B G_{2} \leq B D$ and $D$ has a unique cyclic subgroup of order 4 , we see that $B G_{2}=B C$. By the first paragraph, there is an element $s \in N_{S_{4}}(C)=D$ such that $\left(B \cap G_{1}\right)^{s}=B \cap G_{2}$. That is, $B \cap G=B \cap H$ as claimed. 


\section{Chapter 3}

\section{Cohomology of group extensions}

Given $T=V_{4}, C$ or $D$, and a finite $T$-submodule $U$ of $B$, we want to construct (up to $G L(4)$-conjugacy) all extensions of $U$ by $T$ in $B S_{4}$. A preliminary step in a solution to this extension problem is to calculate the order of the second cohomology group of $T$ with coefficients in $U$. In this chapter, we set up the cohomological machinery required for this step. We give properties of the Lyndon-Hochschild-Serre spectral sequence and show how knowledge of differentials of this spectral sequence may be used to calculate the orders of low-dimensional cohomology groups. Explicit descriptions of these differentials are given, both in a general context and for a specific example of relevance to the main problem of the thesis.

\subsection{Cohomology theory of finite groups}

Most of the material in this section and some in the next is standard, although it does not appear in a single reference nor always in the language that we will use. Also, concepts in the sequel depend on the definitions of low-dimensional cohomology groups in terms of cocycles and their subsequent group-theoretic characterisations. These are given explicitly. Any of the cohomology texts cited in the bibliography is a reference for unproved statements.

Throughout this section, fix a group $G$ and a (right) $G$-module $U$. That is, $U$ is an abelian group with a specified homomorphism of $G$ into $\operatorname{Aut}(U)$. A $G$-complex $\mathbf{P}$ is a sequence of $G$-modules $P_{i}$, where $P_{i}=0$ if $i<0$, together with a sequence of $G$-homomorphisms $d_{i}: P_{i} \rightarrow P_{i-1}$, called differentials, such that $d_{i} d_{i-1}=0$. For $n \geq 0$, the $n$th homology group of the $G$-complex $\mathbf{P}$ is the abelian group 
$\operatorname{ker} d_{n} / \operatorname{im} d_{n+1}$. A $G$-projective resolution of a $G$-module $M$ is a $G$-complex $\mathbf{P}$ of projective modules together with an "augmentation map" $\varepsilon: P_{0} \rightarrow M$, such that the augmented complex

$$
\cdots \stackrel{d_{i+1}}{\rightarrow} P_{i} \stackrel{d_{i}}{\rightarrow} P_{i-1} \stackrel{d_{i-1}}{\rightarrow} \cdots \stackrel{d_{2}}{\rightarrow} P_{1} \stackrel{d_{1}}{\rightarrow} P_{0} \stackrel{\varepsilon}{\rightarrow} M \rightarrow 0
$$

is exact. We denote this $G$-projective resolution by $\mathbf{P} \stackrel{\varepsilon}{\rightarrow} M$. Every $G$-module has at least one $G$-projective resolution.

Let $U_{1}$ and $U_{2}$ be $G$-modules. For $\phi \in \operatorname{Hom}\left(U_{1}, U_{2}\right)$ and $g \in G$, define $\phi \circ g$ by

$$
u(\phi \circ g)=\left(u g^{-1}\right) \phi g
$$

This turns $\operatorname{Hom}\left(U_{1}, U_{2}\right)$ into a $G$-module. It is traditional to denote the subgroup of $G$-fixed points of $U$ by $U^{G}$, and the group of $\mathbb{Z} G$-homomorphisms between $U_{1}$ and $U_{2}$ by $\operatorname{Hom}_{G}\left(U_{1}, U_{2}\right)$. By definition, $\operatorname{Hom}_{G}\left(U_{1}, U_{2}\right)=\operatorname{Hom}\left(U_{1}, U_{2}\right)^{G}$.

Now we define the cohomology groups of $G$ with coefficients in $U$. Regard $\mathbb{Z}$ as a trivial $G$-module and choose a $G$-projective resolution $\mathbf{P} \stackrel{\varepsilon}{\rightarrow} \mathbb{Z}$. If $d_{i}: P_{i} \rightarrow P_{i-1}$ is the $i$ th differential of $\mathbf{P}$, we define $d_{i}^{*}: \operatorname{Hom}_{G}\left(P_{i-1}, U\right) \rightarrow \operatorname{Hom}_{G}\left(P_{i}, U\right)$ by $f d_{i}^{*}=d_{i} f$ for $f \in \operatorname{Hom}_{G}\left(P_{i-1}, U\right)$. By applying $\operatorname{Hom}(-, U)$ to $\mathbf{P}$, we obtain the complex $\operatorname{Hom}_{G}(\mathbf{P}, U)$ :

$0 \rightarrow \operatorname{Hom}_{G}\left(P_{0}, U\right) \stackrel{d_{1}^{*}}{\rightarrow} \operatorname{Hom}_{G}\left(P_{1}, U\right) \stackrel{d_{2}^{*}}{\rightarrow} \ldots \stackrel{d_{i-1}^{*}}{\rightarrow} \operatorname{Hom}_{G}\left(P_{i-1}, U\right) \stackrel{d_{i}^{*}}{\rightarrow} \operatorname{Hom}_{G}\left(P_{i}, U\right) \stackrel{d_{i+1}^{*}}{\rightarrow} \cdots$

The $n$th cohomology group of $G$ with coefficients in $U$, denoted $H^{n}(G, U)$, is the $n$th homology group $\operatorname{ker} d_{n+1}^{*} / \operatorname{im} d_{n}^{*}$ of $\operatorname{Hom}_{G}(\mathbf{P}, U)$. The isomorphism type of the cohomology groups does not depend on the particular $G$-projective resolution of $\mathbb{Z}$ chosen.

A function $\psi: G \times \ldots \times G \rightarrow U$ such that $\left(g_{1}, \ldots, g_{n}\right) \psi=0$ if some $g_{i}=1$, is called a (normalised) $n$-cochain. For the so-called "standard" $G$-projective resolution $\mathbf{P} \stackrel{\varepsilon}{\rightarrow} \mathbb{Z}\left[\right.$ which from now on we assume is used to define the $\left.H^{i}(G, U)\right]$, the set $\operatorname{Hom}_{G}\left(P_{n}, U\right)$ is in one-to-one correspondence with the set of $n$-cochains from $G$ to $U$. The action of $d_{n+1}^{*}$ on $n$-cochains is given by

$$
\begin{aligned}
\left(g_{1}, g_{2}, \ldots, g_{n+1}\right) \psi d_{n+1}^{*}= & \left(g_{2}, \ldots, g_{n+1}\right) \psi-\left(g_{1} g_{2}, g_{3}, \ldots, g_{n+1}\right) \psi \\
& +\left(g_{1}, g_{2} g_{3}, \ldots, g_{n+1}\right) \psi-\ldots \\
& +(-1)^{i}\left(g_{1}, \ldots, g_{i} g_{i+1}, \ldots, g_{n+1}\right) \psi+\cdots \\
& +(-1)^{n+1}\left(g_{1}, \ldots, g_{n}\right) \psi g_{n+1}
\end{aligned}
$$


Note that $\psi d_{n+1}^{*}$ is certainly an $(n+1)$-cochain. The elements of $\operatorname{ker} d_{n+1}^{*}$ are called $n$-cocycles and the elements of $\operatorname{im} d_{n}^{*}$ are called $n$-coboundaries.

The next result shows how we may construct an exact sequence of cohomology groups from an exact sequence of coefficient modules.

Proposition 3.1.1 Suppose that

$$
0 \rightarrow L \rightarrow M \rightarrow N \rightarrow 0
$$

is an exact sequence of $G$-modules. Then we have a cohomology long exact sequence:

$$
0 \rightarrow H^{0}(G, L) \rightarrow H^{0}(G, M) \rightarrow H^{0}(G, N) \rightarrow H^{1}(G, L) \rightarrow H^{1}(G, M) \rightarrow \cdots
$$

Proof. See Hilton \& Stammbach (1971), p.189, 2.3.

Except for the connecting homomorphisms $H^{i}(G, N) \rightarrow H^{i+1}(G, L)$, the homomorphisms in the cohomology long exact sequence of Proposition 3.1 .1 are induced from those in the short exact sequence of modules.

Now we give in detail some well-known characterisations of the cohomology groups $H^{i}(G, U), i \leq 3$.

Of course, 0 -cochains from $G$ to $U$ are just elements of $U$. Since the contravariant functor $\operatorname{Hom}_{G}(-, U)$ is left exact, we have an exact sequence

$$
0 \rightarrow \operatorname{Hom}_{G}(\mathbb{Z}, U) \stackrel{\varepsilon^{*}}{\rightarrow} \operatorname{Hom}_{G}\left(P_{0}, U\right) \stackrel{d_{1}^{*}}{\rightarrow} \operatorname{Hom}_{G}\left(P_{1}, U\right) \stackrel{d_{2}^{*}}{\rightarrow} \cdots
$$

Thus $\operatorname{ker} d_{1}^{*}=\operatorname{im} \varepsilon^{*}=\operatorname{Hom}_{G}(\mathbb{Z}, U)=\operatorname{Hom}(\mathbb{Z}, U)^{G} \cong U^{G}$ and so $H^{0}(G, U)=U^{G}$.

The first cohomology group of $G$ with coefficients in $U$ has a natural description in terms of derivations from $G$ to $U$. By (3.1), a 1-cocycle $\delta$ from $G$ to $U$ satisfies

$$
(g h) \delta=g \delta h+h \delta
$$

for all $g, h \in G$. The 1-cocycles are called (right) derivations and the abelian group consisting of the derivations from $G$ to $U$ is denoted $\operatorname{Der}(G, U)$. In the degenerate case $n=0$, the interpretation of (3.1) is that the 1 -coboundaries from $G$ to $U$ are derivations $\delta_{u}$, one for each $u \in U$, where

$$
g \delta_{u}=u(1-g)
$$

We call $\delta_{u}$ an inner derivation from $G$ to $U$ and denote the subgroup of inner derivations from $G$ to $U$ by $\operatorname{Ider}(G, U)$. Thus $H^{1}(G, U)$ is identified with the 
abelian group $\operatorname{Der}(G, U) / \operatorname{Ider}(G, U)$. Other characterisations of $H^{1}(G, U)$ will be needed and these are given next.

Consider the semidirect product $U \rtimes G$. Conjugacy in $U \rtimes G$ is an equivalence relation on the set of complements of $U$ in $U \rtimes G$. If $K$ is a complement of $U$ in $U \rtimes G$ then define $\delta: G \rightarrow U$ as follows: for $g \in G$, write $g=k u^{-1}$ for some $k \in K$ and $u \in U$, and set $g \delta=u$. It may be verified that $\delta \in \operatorname{Der}(G, U)$. Conversely, given $\delta \in \operatorname{Der}(G, U)$, the following is a complement of $U$ in $U \rtimes G$, with corresponding derivation $\delta$ :

$$
\{g(g \delta) \mid g \in G\}
$$

Thus, we have the familiar group-theoretic characterisation of $H^{1}(G, U)$, as given in the next theorem.

Theorem 3.1.2 There is a one-to-one correspondence between $\operatorname{Der}(G, U)$ and the set of complements of $U$ in the semidirect product $U \rtimes G$. If two such complements are conjugate in $U \rtimes G$ then they correspond to derivations that differ by at most an inner derivation. That is, $H^{1}(G, U)$ is in one-to-one correspondence with the set of conjugacy classes of complements of $U$ in $U \rtimes G$.

The second cohomology group may be described in terms of group extensions. Let $K$ be a group, written additively, though not necessarily abelian. An extension of $K$ by $G$ is a short exact sequence of groups

$$
0 \rightarrow K \stackrel{\iota}{\rightarrow} E \stackrel{\pi}{\rightarrow} G \rightarrow 1
$$

Often we refer to the group $E$ as an extension of $K$ by $G$ when $\iota$ and $\pi$ are understood. It is customary to identify $K$ with $K \iota$. There is a natural equivalence relation on the set of extensions of $K$ by $G$. Two extensions $1 \rightarrow K \stackrel{\iota_{1}}{\rightarrow} E_{1} \stackrel{\pi_{1}}{\rightarrow} G \rightarrow 1$ and $1 \rightarrow K \stackrel{\iota_{2}}{\rightarrow} E_{2} \stackrel{\pi_{2}}{\rightarrow} G \rightarrow 1$ are said to be equivalent if there is a homomorphism $\theta: E_{1} \rightarrow E_{2}$ such the diagram

$$
\begin{array}{ccccccc}
0 \rightarrow & K & \stackrel{\iota_{1}}{\rightarrow} & E_{1} & \stackrel{\pi_{1}}{\rightarrow} & G & \rightarrow 1 \\
\| & & \downarrow \theta & & \| & \\
0 \rightarrow & K & \stackrel{\iota_{2}}{\rightarrow} & E_{2} & \stackrel{\pi_{2}}{\rightarrow} & G & \rightarrow 1
\end{array}
$$

commutes. The equivalence $\theta$ is automatically an isomorphism. However, note that isomorphic extensions need not be equivalent.

The extension (3.2) gives rise to a homomorphism $\chi: G \rightarrow \operatorname{Out}(K)$ in the following way. First choose a transversal function $\sigma: G \rightarrow E$. That is, $\sigma$ is a set map 
such that $\sigma \pi$ is the identity on $G$. We also stipulate that $1 \sigma=1$ (normalisation). Let $\mu: E \rightarrow \operatorname{Inn}(E)$ be the homomorphism which maps each element of $E$ to the inner automorphism of $E$ it induces. Then for each $g \in G$ set

$$
g \chi=g \sigma \mu \operatorname{Inn}(K)
$$

As defined, $\chi$ is a homomorphism of $G$ into $\operatorname{Out}(K)$, independent of the choice of $\sigma$. This homomorphism reflects the way that $\zeta(K)$, embedded as a normal abelian subgroup of $E$ via $\iota$, is a $G$-module under the conjugation action of elements of $E$. A homomorphism of $G$ into $\operatorname{Out}(K)$, not necessarily arising in this way, is called a coupling of $G$ to $K$. Equivalent extensions have the same coupling. A coupling $\chi$ of $G$ to $K$ prescribes a $G$-module structure for $\zeta(K)$ as follows. For each $g \in G$, choose a representative $g \lambda$ of $g \chi \in \operatorname{Aut}(K) / \operatorname{Inn}(K)$ and set

$$
z g=(z) g \lambda
$$

for $z \in \zeta(K)$. In fact, giving a $G$-module structure for an abelian group is equivalent to giving a coupling of $G$ to that group.

Every extension of $K$ by $G$ gives rise to a coupling of $G$ to $K$, but not every coupling arises from an extension. Given $K, G$ and a coupling $\chi$ from $G$ to $K$, the group extension problem is to construct all extensions of $K$ by $G$ with coupling $\chi$. Of course, such extensions need not exist. When $K=U$ is a $G$-module and $\chi$ prescribes the $G$-module structure of $U, \chi$ arises from the split extension $U \rtimes_{\chi} G$. The group extension problem in this case is to construct all extensions of $U$ by $G$ with the same coupling $\chi$ as that given by the action of $G$ on $U$ (note that $\operatorname{Inn}(K)=1$ in this case). This usually means listing representatives of isomorphism classes of such extensions. As we noted above, isomorphism does not imply equivalence. This is a typical source of difficulty in applying cohomology theory to group extension problems.

Given the extension (3.2) of $K$ by $G$ and a normalised transversal function $\sigma: G \rightarrow E$, for $x, y \in G$ choose $(x, y) \psi \in K$ such that

$$
x \sigma y \sigma=(x y) \sigma(x, y) \psi
$$

Observe that $(1, y) \psi=(x, 1) \psi=0$. A familiar calculation, using associativity in $G$, shows that

$$
(x, y z) \psi+(y, z) \psi=(x y, z) \psi+((x, y) \psi)^{z \sigma}
$$


for all $x, y, z \in G$. If $K$ is abelian, then by (3.1), $\psi$ is a 2-cocycle from $G$ to $K$, where the $G$-module structure of $K$ is prescribed by the coupling of $G$ to $K$ arising from (3.2). So assume now that $K=U$ is a $G$-module,

$$
0 \rightarrow U \stackrel{\iota}{\rightarrow} E \stackrel{\pi}{\rightarrow} G \rightarrow 1
$$

is an extension of $U$ by $G, \sigma$ is a normalised transversal function from $G$ to $U$, and the coupling $\chi$ arising from (3.4) is the same as that given by the action of $G$ on $U$. (This last condition is equivalent to: $U$, embedded in $E$ by $\iota$, is a $G$-module under the action induced from conjugation by elements of $E$.) Also, let $\psi$ be the 2-cocycle from $G$ to $U$ arising from $\sigma$. If a different transversal function is chosen, the above procedure yields a 2-cocycle in the same cohomology class as $\psi$. Therefore, there is a well-defined map from the set of extensions of $U$ by $G$ whose couplings prescribe the action of $G$ on $U$, to $H^{2}(G, U)$. Conversely, given a 2-cocycle $\phi$ from $G$ to $U$, define a composition on the set of pairs

$$
E^{\prime}=\{(g, u) \mid g \in G, u \in U\}
$$

by

$$
\left(g_{1}, u_{1}\right)\left(g_{2}, u_{2}\right)=\left(g_{1} g_{2}, u_{1} g_{2}+u_{2}+\left(g_{1}, g_{2}\right) \phi\right) .
$$

This composition is associative, with identity $(1,0)$, and $(g, u) \in E^{\prime}$ has inverse

$$
\left(g^{-1},-\left(g, g^{-1}\right) \phi-u g^{-1}\right)
$$

Thus $E^{\prime}$ is an extension of $U$ by $G$ and any coupling arising from this extension is the same as that given by the action of $G$ on $U$. Moreover, when $\phi$ arises from the extension (3.4), these two extensions are equivalent. (Hence, in explicit calculations involving an extension of $U$ by $G$, we will sometimes use this equivalent formulation.) These facts are summarised in the statement of the next theorem, a proof of which may be found in Mac Lane (1963), p.112, Theorem 4.1.

Theorem 3.1.3 There is a one-to-one correspondence between $H^{2}(G, U)$ and the set of equivalence classes of extensions (3.4) of $U$ by $G$ with the same coupling as that given by the action of $G$ on $U$.

Clearly, then, the order of $H^{2}(G, U)$ provides an upper bound on the number of isomorphism classes of extensions (3.4) of $U$ by $G$. 
The zero element of $H^{2}(G, U)$ is the equivalence class of the split extension of $U$ by $G$. For later reference, we point out the connection between transversal homomorphisms and derivations in this case. Suppose that (3.4) splits. That is, there is a transversal homomorphism $\sigma: G \rightarrow U$. Moreover, if $\tau$ is another transversal homomorphism then for each $g \in G$ there is $u_{g} \in U$ such that $g \tau=g \sigma . u_{g}$. The map $\delta$ that sends $g$ to $u_{g}$ is in fact a derivation from $G$ to $U$. We have

$$
g \tau=g \sigma . g \delta \iota
$$

Conversely, given $\delta \in \operatorname{Der}(G, U)$ and a transversal homomorphism $\sigma$, the $\tau$ defined by $(3.5)$ is also a transversal homomorphism from $G$ to $U$.

Before discussing third cohomology, we outline the relationship between automorphisms of $E$, and automorphisms of $U$ and $G$, thereby giving a characterisation of derivations in terms of such automorphisms. These results are needed in Section 3.2. Let $\theta$ be an automorphism of $E$ leaving $U$ setwise invariant. Then there are obvious induced automorphisms $\theta_{U}=\left.\theta\right|_{U}$ of $U$ (more rigorously, $\theta_{U}=\iota \iota_{\iota}{ }^{-1}$ ) and $\theta_{G}$ of $G$ defined for $x \in G$ by

$$
x \theta_{G}=x \sigma \theta \pi .
$$

Clearly, the definition of $\theta_{G}$ depends only on $\pi$, not on $\sigma$. Given automorphisms of $G$ and $U$, we would like to know if these are induced from an element of $N_{\operatorname{Aut}(E)}(U)$. The next proposition gives necessary and sufficient conditions for this situation to occur.

Proposition 3.1.4 Let $\gamma \in \operatorname{Aut}(G)$ and $\nu \in \operatorname{Aut}(U)$. Then there is $\theta \in N_{\text {Aut }(E)}(U)$ such that $\theta_{G}=\gamma$ and $\theta_{U}=\nu$ if and only if there is a 1 -cochain $\phi$ from $G$ to $U$ such that

$$
(x, y) \psi \nu=(x \gamma, y \gamma) \psi+(x \gamma, y \gamma) \phi d_{2}^{*}
$$

and

$$
x \chi \cdot \nu=\nu \cdot x \gamma \chi
$$

for all $x, y, z \in G$. Given $\gamma, \nu$ and any $\phi$ satisfying the stated conditions, $\theta \in N_{\mathrm{Aut}(E)}(U)$ defined for $x \in G$ and $u \in U$ by

$$
(x, u) \theta=(x \gamma, u \nu+x \gamma \phi)
$$

satisfies $\theta_{G}=\gamma$ and $\theta_{U}=\nu$. 
Proof. The proof is straightforward and is given in Robinson (1981), p.67, 4.1.

In Robinson (1981), the pair $(\gamma, \nu)$ satisfying the conditions of Proposition 3.1.4 is called inducible. Given an inducible pair $(\gamma, \nu)$, there may be many possibilities for $\theta$, depending on the choice of $\phi$. We remark that if $\theta \in N_{\operatorname{Aut}(E)}(U)$, then $\gamma=\theta_{G}$ and $\nu=\theta_{U}$ clearly satisfy the second condition of Proposition 3.1.4, called compatibility.

Proposition 3.1.5 In the notation above, there is an isomorphism of $\operatorname{Der}(G, U)$ onto the group

$$
\left\{\theta \in N_{\text {Aut }(E)}(U) \mid \theta_{G}=\operatorname{id}_{G}, \theta_{U}=\mathrm{id}_{U}\right\} .
$$

Under this isomorphism, the inner derivation from $G$ to $U$ induced by the element $u \in U$ is mapped to the inner automorphism of $E$ induced by $u$.

Proof. See Robinson (1981), p.66. Take $\phi=\delta \in \operatorname{Der}(G, U), \gamma=\mathrm{id}_{G}, \nu=\mathrm{id}_{U}$ in Proposition 3.1.4. Then with these choices, the hypotheses of Proposition 3.1.4 are satisfied and thus we have an isomorphism $\alpha_{\delta} \in N_{\operatorname{Aut}(E)}(U)$ which is defined for $e=(g, u) \in E$ by

$$
\begin{aligned}
e \alpha_{\delta} & =(g, u+g \delta) \\
& =(g, u)(1, g \delta) \\
& =e \cdot e \pi \delta
\end{aligned}
$$

Conversely, suppose that $\alpha \in N_{\operatorname{Aut}(E)}(U)$ induces the identity on both $G$ and $U$. If $g \in G$, choose $g \sigma=(g, u) \in E$. By Proposition 3.1.4, $g \sigma \alpha=g \sigma .\left(1, g \delta_{\alpha}\right)$ for some $\delta_{\alpha} \in \operatorname{Der}(G, U)$. That is,

$$
g \delta_{\alpha}=(g \sigma)^{-1} \cdot g \sigma \alpha
$$

The homomorphisms $\delta \mapsto \alpha_{\delta}$ and $\alpha \mapsto \delta_{\alpha}$ are clearly inverse to each other. The last assertion is also obvious.

Recall that every extension (3.2) gives rise to a coupling of $G$ to $K$, but the converse is not necessarily true. The group-theoretic characterisation of third cohomology provides a condition for the converse to be true, as we now show.

Let $\chi$ be a coupling of $G$ to $K$. Define the set map $\lambda: G \rightarrow \operatorname{Aut}(K)$ by choosing for each $g \in G$ a representative $g \lambda$ of the coset $g \chi$, ensuring that $1 \lambda=1$. Then for $x, y \in G$, we may choose $(x, y) \psi \in K$ such that $(x, 1) \psi=(1, y) \psi=0$ and

$$
x \lambda . y \lambda=(x y) \lambda .(x, y) \psi \mu
$$


Proceeding from this definition of $\psi$, and by the same calculation as that leading to $(3.3)$, we have

$$
((x, y z) \psi+(y, z) \psi) \mu=((x y, z) \psi+((x, y) \psi) z \lambda) \mu
$$

for all $x, y, z \in G$. Since the kernel of $\mu$ on $K$ is $\zeta(K)$, there is $(x, y, z) \kappa \in \zeta(K)$ such that

$$
(x, y z) \psi+(y, z) \psi=(x y, z) \psi+((x, y) \psi) z \lambda+(x, y, z) \kappa .
$$

Using this identity, it may be verified that in fact $\kappa$ is a 3-cocycle from $G$ to $\zeta(K)$, called an obstruction (determined by the coupling $\chi$ ). For different choices of $\lambda$ and $\psi, \kappa$ is changed only by a 3 -coboundary; hence, to each coupling of $G$ to $K$ we associate a unique element of $H^{3}(G, \zeta(K))$. If $\chi$ arises from an extension of $K$ by $G$, then (3.3) shows that the cohomology class of an obstruction determined by $\chi$ is the zero element of $H^{3}(G, \zeta(K))$. In fact, the converse is also true.

Theorem 3.1.6 Let $\chi$ be a coupling of $G$ to $K$. Then $\chi$ arises from an extension of $K$ by $G$ if and only if the cohomology class of an obstruction determined by $\chi$ is the zero element of $H^{3}(G, \zeta(K))$, where the $G$-module structure of $\zeta(K)$ is prescribed by $\chi$.

Proof. See Mac Lane (1963), p.128, Theorem 8.7.

This completes the description of low-dimensional cohomology. We conclude this section with Shapiro's Lemma and the cohomology of finite cyclic groups.

Let $H$ be a subgroup of $G$ and $U$ a $H$-module. Define the following $G$-modules:

$$
\operatorname{Ind}_{H}^{G} U=U \otimes_{H} \mathbb{Z} G
$$

and

$$
\operatorname{Coind}_{H}^{G} U=\operatorname{Hom}_{H}(\mathbb{Z} G, U)
$$

Ind ${ }_{H}^{G} U$ is induced from $H$ to $G$ and Coind $_{H}^{G} U$ is coinduced from $H$ to $G$. The module Ind ${ }_{H}^{G} U$ is the direct sum of the abelian groups $U \otimes g$, where $g$ runs over a transversal of the (right) cosets of $H$ in $G$. Therefore, the action of $G$ on $\operatorname{Ind}_{H}^{G} U$ is to permute transitively the summands $U \otimes g$.

Proposition 3.1.7 Suppose that $H$ has finite index in $G$. Then

$$
\operatorname{Ind}_{H}^{G} U \cong \operatorname{Coind}_{H}^{G} U
$$


Also,

$$
H^{n}\left(G, \operatorname{Ind}_{H}^{G} U\right) \cong H^{n}(H, U)
$$

for $n \geq 1$.

Proof. See Brown (1982), p.70, 5.9 and p.73, 6.2.

The cohomology of finite cyclic groups is well-known and is described in the next proposition.

Proposition 3.1.8 Let $G$ be a cyclic group of order $n$ with generator $g$, and $U$ a $G$-module. Then multiplication by $1-g$ and $1+g+\cdots+g^{n-1}$ are $G$-endomorphisms of $U$ and for $i \geq 1$,

$$
\begin{aligned}
& H^{2 i-1}(G, U) \cong \operatorname{ker}\left(1+g+\cdots+g^{n-1}\right) / U(1-g) \\
& H^{2 i}(G, U) \cong \operatorname{ker}(1-g) / U\left(1+g+\cdots+g^{n-1}\right) .
\end{aligned}
$$

Proof. See Hilton \& Stammbach (1971), p.200, §7, for example.

Remark 3.1.9 If $U$ is finite in Proposition 3.1.8, then $\left|H^{i}(G, U)\right|=\left|H^{i+1}(G, U)\right|$ for all $i \geq 1$.

Remark 3.1.10 For $G$ in Proposition 3.1.8, the map $\alpha$ defined for $\delta \in \operatorname{Der}(G, U)$ by

$$
(\delta) \alpha=g \delta
$$

induces an isomorphism of $H^{1}(G, U)$ onto $\operatorname{ker}\left(1+g+\cdots+g^{n-1}\right) / U(1-g)$. For by induction,

$$
\left(g^{k}\right) \delta=g \delta+(g \delta) g+\cdots+(g \delta) g^{k-1},
$$

$1 \leq k \leq n$. Therefore $g \delta \in \operatorname{ker}\left(1+g+\cdots+g^{n-1}\right)$, and two derivations are the same if and only if they agree on the generator $g$ of $G$. Note also that $\operatorname{Ider}(G, U)$ is mapped onto $U(1-g)$ by $\alpha$.

Conversely, given $u \in \operatorname{ker}\left(1+g+\cdots+g^{n-1}\right)$, define the map $u \beta=d: G \rightarrow U$ by

$$
\begin{aligned}
g d & =u \\
g^{2} d & =u(g+1) \\
& \vdots \\
g^{k} d & =u\left(g^{k-1}+\cdots+g+1\right),
\end{aligned}
$$

for $k>1$. Then $d$ is a derivation from $G$ to $U$ and $U(1-g)$ is mapped onto $\operatorname{Ider}(G, U)$ by $\beta$. It is easy to see that $\alpha$ and $\beta$ are inverse isomorphisms. 


\subsection{The LHS spectral sequence}

The Lyndon-Hochschild-Serre (LHS) spectral sequence relates cohomology of a group $G$ to cohomology of a normal subgroup $N$ and the factor group $G / N$. This spectral sequence is the major tool that we will use to reduce in difficulty the extension problem for finite $V_{4}$ - and $D$-submodules of $B$. In this section we give the relevant properties of the LHS spectral sequence. The reference is Mac Lane (1963).

Let $R$ be a ring with 1. A graded $R$-module $M$ is just a family $M=\left\{M_{n}\right\}$ of $R$-modules, $n \in \mathbb{Z}$. A filtration $F$ of $M$ is a nested sequence of modules $\cdots \supseteq F^{p} M_{n} \supseteq F^{p+1} M_{n} \supseteq \cdots$ for each $n$ (a superscript $p$ here or anywhere else in this chapter does not necessarily denote a prime).

A (cohomology) spectral sequence consists of a sequence of bigraded $R$-modules $E_{r}=\left\{E_{r}^{p, q}\right\}, r \geq 2$, where $E_{r}^{p, q}=0$ if $p<0$ or $q<0$, together with differentials $d_{r}^{p, q}: E_{r}^{p, q} \rightarrow E_{r}^{p+r, q-r+1}$ such that $d_{r}^{p-r, q+r-1} d_{r}^{p, q}=0$ and

$$
E_{r+1}^{p, q} \cong \operatorname{ker} d_{r}^{p, q} / \operatorname{im} d_{r}^{p-r, q+r-1}
$$

That is, $E_{r+1}$ is the bigraded homology module of $E_{r}$ with respect to $d_{r}=\left\{d_{r}^{p, q}\right\}$. In general, however, $d_{r+1}$ is not determined by $E_{r}$ or $d_{r}$.

For fixed $p$ and $q$, there is some $r$ such that $E_{r}^{p, q}=E_{r+1}^{p, q}=E_{r+2}^{p, q}=\ldots$; certainly $r>m=\max \{p, q+1\}$ suffices. Denote the limit term $E_{m+1}^{p, q}$ by $E_{\infty}^{p, q}$. Since $E_{r+1}^{p, 0} \cong E_{r}^{p, 0} / \mathrm{im} d_{r}^{p-r, r-1}$, there is a sequence of epimorphisms

$$
E_{2}^{p, 0} \rightarrow E_{3}^{p, 0} \rightarrow \cdots \rightarrow E_{\infty}^{p, 0}=E_{p+1}^{p, 0}
$$

The composite of these epimorphisms is called an edge homomorphism (on the base). Similarly, there is a sequence of monomorphisms

$$
E_{\infty}^{0, q}=E_{q+2}^{0, q} \rightarrow E_{q+1}^{0, q} \rightarrow \cdots \rightarrow E_{2}^{0, q}
$$

The composite of these monomorphisms is also called an edge homomorphism (on the fibre).

The spectral sequence $\left\{E_{r}, d_{r}\right\}$ converges to a graded module $H=\left\{H^{n}\right\}$, $H^{n}=0$ if $n<0$, if there is a filtration $F$ of $H$

$$
H^{n}=F^{0} H^{n} \supseteq F^{1} H^{n} \supseteq \cdots \supseteq F^{n} H^{n} \supseteq F^{n+1} H^{n}=0
$$

such that

$$
E_{\infty}^{p, q} \cong F^{p} H^{p+q} / F^{p+1} H^{p+q}
$$


Fix a $G$-module $U$. The abelian groups $H^{n}(N, U)$ become $G$-modules, in fact $G / N$-modules, under the following action of $G$ on cohomology classes of $n$-cocycles. If $\psi$ is an $n$-cocycle from $N$ to $U$ and $g \in G$, define the $n$-cocycle $\psi \circ g$ by

$$
\left(x_{1}, \ldots, x_{n}\right)(\psi \circ g)=\left(x_{1}^{g^{-1}}, \ldots, x_{n}^{g^{-1}}\right) \psi \cdot g
$$

for $x_{i} \in N$. Note by (3.1) that if $\psi$ is an $n$-coboundary, then $\psi \circ g$ is also an $n$ coboundary. Hence, (3.6) gives a well-defined action of $G$ on $H^{n}(N, U)$ by setting $[\psi] \circ g=[\psi \circ g]$, where $[-]$ denotes cohomology class. Let $P_{n}$ be the $n$th term in the standard $G$-projective resolution of $\mathbb{Z}$. Under the correspondence between $\operatorname{Hom}_{N}\left(P_{n}, U\right)$ and the set of $n$-cochains from $N$ to $U$, this action corresponds to the action $\circ$ of $G$ on elements of $\operatorname{Hom}\left(P_{n}, U\right)$. In particular, $N$ acts trivially on $H^{n}(N, U)$ and so there is an action of $G / N$ on $H^{n}(N, U)$ induced by $\circ$. With this action we define the cohomology groups $H^{p}\left(G / N, H^{q}(N, U)\right)$ for all $p, q \geq 0$.

Remark 3.2.1 Let $N$ be finite cyclic. By Proposition 3.1.8, the $H^{n}(N, U)$ are isomorphic, as abelian groups, to sections of the $G$-module $U$. Thus, there is an action of $G / N$ on $H^{n}(N, U)$ corresponding to the action of $G$ on sections of $U$. We emphasise that, in general, this is not the same action as that given by (3.6).

The only spectral sequence that we will use in the applications is the LyndonHochschild-Serre spectral sequence. This will not be defined. Only its important properties, given in the next theorem, will be used.

Theorem 3.2.2 Let $G, N, U$ be as above, and let $G$ act on $H^{q}(N, U)$ as in (3.6). Then there is a spectral sequence $\left\{E_{r}, d_{r}\right\}$, called the Lyndon-Hochschild-Serre spectral sequence, such that

- $E_{2}^{p, q} \cong H^{p}\left(G / N, H^{q}(N, U)\right)$,

- $\left\{E_{r}, d_{r}\right\}$ converges to the graded module $H^{p+q}(G, U)$.

Proof. See Mac Lane (1963), p.351, Theorem 10.1.

When we refer to Theorem 3.2.2 in the sequel, we assume convergence via some fixed filtration $F$.

In applications involving the LHS spectral sequence, one chooses $N \unlhd G$ such that the $G / N$-module $H^{q}(N, U)$ is known, and hence the spectral sequence terms $E_{2}^{p, q}$ are calculable. One also needs to understand the action of various differentials. 
The differentials may be defined in several contexts: on cocycles; for the grouptheoretic interpretations of low-dimensional cohomology (given in Section 3.1); and, in the case that $G$ is finite and $N, G / N$ are cyclic, on sections of $U$. Generally speaking, it is difficult to define differentials on cocycles by proceeding directly from the definition of the LHS spectral sequence. In Section 3.3, we give group-theoretic interpretations of the differentials that are relevant to our situation.

Before indicating precisely which of the LHS spectral sequence differentials we will need to understand, we discuss edge homomorphisms and show how they are related to the filtration $F$.

There is a natural inflation homomorphism, inf: $H^{n}\left(G / N, U^{N}\right) \rightarrow H^{n}(G, U)$. This is defined for each $n$-cocycle $\psi$ from $G / N$ to $U^{N}$ by

$$
\left(g_{1}, \ldots, g_{n}\right)(\psi \mathrm{inf})=\left(g_{1} N, \ldots, g_{n} N\right) \psi
$$

Note that inf maps $n$-coboundaries to $n$-coboundaries and that the codomain of inf is actually $H^{n}\left(G, U^{N}\right)$, embedded in $H^{n}(G, U)$. In fact, inf becomes a homomorphism by setting $[\psi] \inf =[\psi \inf ]$. This inflation homomorphism is injective for $n=1$ but not necessarily for $n>1$.

The restriction homomorphism, res: $H^{n}(G, U) \rightarrow H^{n}(N, U)$, is obtained by restricting to $N$ the domain of definition of $n$-cocycles from $G$ to $U$. Since the action of $G$ on $H^{n}(G, U)$ induced by (3.6) is trivial, the image of res lies in $H^{n}(N, U)^{G / N}$. It is always true that im inf $\leq$ ker res.

Proposition 3.2.3 For the LHS spectral sequence, the composite of the edge homomorphism

$$
H^{p}\left(G / N, U^{N}\right) \rightarrow E_{\infty}^{p, 0}
$$

on the base, and the embedding of $E_{\infty}^{p, 0}$ in $H^{p}(G, U)$, is the inflation homomorphism. The composite of the edge homomorphism

$$
E_{\infty}^{0, q} \rightarrow H^{q}(N, U)^{G / N}
$$

on the fibre, and the natural surjection of $H^{q}(G, U)$ onto $E_{\infty}^{0, q}$, is the restriction homomorphism. Thus $F^{p} H^{p}$ may be identified with im inf and $F^{1} H^{p}$ with ker res.

Proof. We use the convergence statement of Theorem 3.2.2. Note that $E_{\infty}^{p, 0}=F^{p} H^{p}$ is certainly embedded in $H^{p}(G, U)$; the first composite therefore has image $F^{p} H^{p}$. 
Also, $E_{\infty}^{0, q} \cong H^{q} / F^{1} H^{q}$, and the second composite has kernel $F^{1} H^{q}$. The first two assertions are proved in Mac Lane (1963), p.352, Proposition 10.2, from which the remaining assertions follow.

Consider LHS spectral sequence terms of total degree 1. By Proposition 3.2.3, we have an exact sequence

$$
0 \rightarrow H^{1}\left(G / N, U^{N}\right) \stackrel{\inf }{\rightarrow} H^{1}(G, U) \rightarrow E_{3}^{0,1} \rightarrow 0
$$

where the rightmost nonzero map is natural: $H^{1} / \mathrm{im}$ inf $\cong E_{\infty}^{0,1}=E_{3}^{0,1}$. Furthermore, $E_{3}^{0,1}=\operatorname{ker} d_{2}^{0,1}$ and $E_{2}^{2,0} / \operatorname{im} d_{2}^{0,1} \cong E_{\infty}^{2,0}=F^{2} H^{2}$. By Proposition 3.2.3, we have an exact sequence

$$
0 \rightarrow E_{3}^{0,1} \rightarrow E_{2}^{0,1} \stackrel{d_{2}^{0,1}}{\rightarrow} E_{2}^{2,0} \stackrel{\inf }{\rightarrow} H^{2}
$$

where the leftmost nonzero map is an edge homomorphism on the fibre. Splicing together the exact sequences (3.7) and (3.8) and using Proposition 3.2.3 again, we have the following five term exact sequence:

$$
0 \rightarrow H^{1}\left(G / N, U^{N}\right) \stackrel{\inf }{\rightarrow} H^{1}(G, U) \stackrel{\text { res }}{\rightarrow} H^{1}(N, U)^{G / N} \stackrel{\tau}{\rightarrow} H^{2}\left(G / N, U^{N}\right) \stackrel{\text { inf }}{\rightarrow} H^{2}(G, U)
$$

where $\tau$ is traditional notation for the first transgression; in this case $\tau=d_{2}^{0,1}$. In general, transgression is defined in terms of additive relations between modules (we do not define this concept).

In order to calculate $\left|H^{2}(G, U)\right|$, consider LHS spectral sequence differentials $d_{r}^{p, q}$ of total degree $p+q \leq 2$. There are three such (nonzero) differentials for $r=2$, one for $r=3$ and none for $r \geq 4$. These differentials are pictured in Figure 3.1. In Figure 3.1, an arrow starting at $E_{r}^{p, q}$ represents the differential $d_{r}^{p, q}$. It can be seen easily from these pictures that an arrow with gradient $(1-r) / r$ starting at any other point in the region $p+q \leq 2$ terminates at a point outside the first quadrant, and thus is zero.

By Theorem 3.2.2,

$$
E_{\infty}^{p, 2-p} \cong F^{p} H^{2} / F^{p+1} H^{2}
$$

so that by considering $p=0,1$ and 2 in turn, we see that

$$
E_{\infty}^{0,2} \cong H^{2} / F^{1} H^{2}
$$


and

$$
E_{\infty}^{1,1} \cong F^{1} H^{2} / E_{\infty}^{2,0}
$$

Now $E_{\infty}^{0,2}=E_{4}^{0,2}, E_{\infty}^{1,1}=E_{3}^{1,1}=\operatorname{ker} d_{2}^{1,1}$ and $E_{\infty}^{2,0}=E_{3}^{2,0}$. Therefore, if $G$ and $U$ are finite then

$$
\left|H^{2}(G, U)\right|=\left|E_{4}^{0,2}\right|\left|\operatorname{ker} d_{2}^{1,1}\right|\left|E_{3}^{2,0}\right| .
$$

Furthermore, $E_{3}^{2,0} \cong \operatorname{coker} d_{2}^{0,1}$ and $E_{4}^{0,2}=\operatorname{ker} d_{3}^{0,2}$; hence

$$
\left|H^{2}(G, U)\right|=\left|\operatorname{ker} d_{3}^{0,2}\right|\left|\operatorname{ker} d_{2}^{1,1}\right|\left|\operatorname{coker} d_{2}^{0,1}\right| \text {. }
$$

Note that the differential $d_{3}^{0,2}$ has domain $\operatorname{ker} d_{2}^{0,2}$ and codomain $H^{3}\left(G / N, U^{N}\right) / \operatorname{im} d_{2}^{1,1}$. By Proposition 3.2.3, ker $d_{3}^{0,2}$ is identified with the image of res on $H^{2}(G, U)$ and ker $d_{2}^{1,1}$ with ker res/im inf.

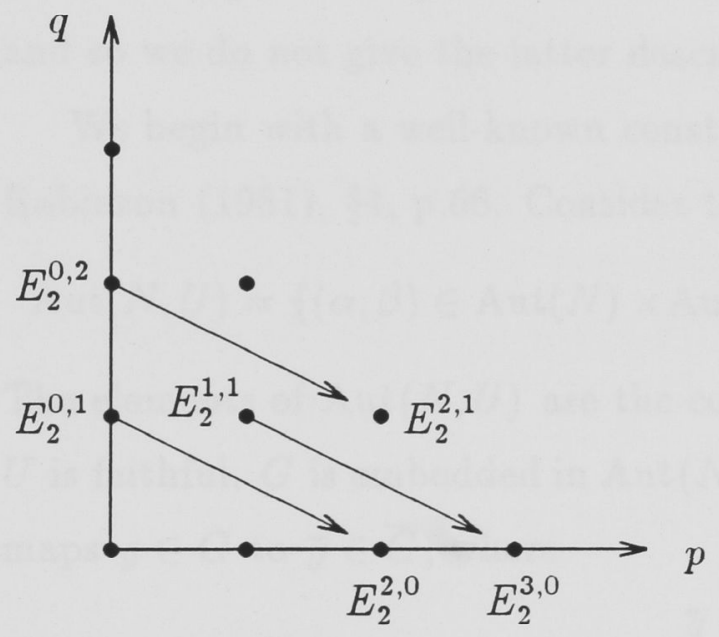

$E_{2}$

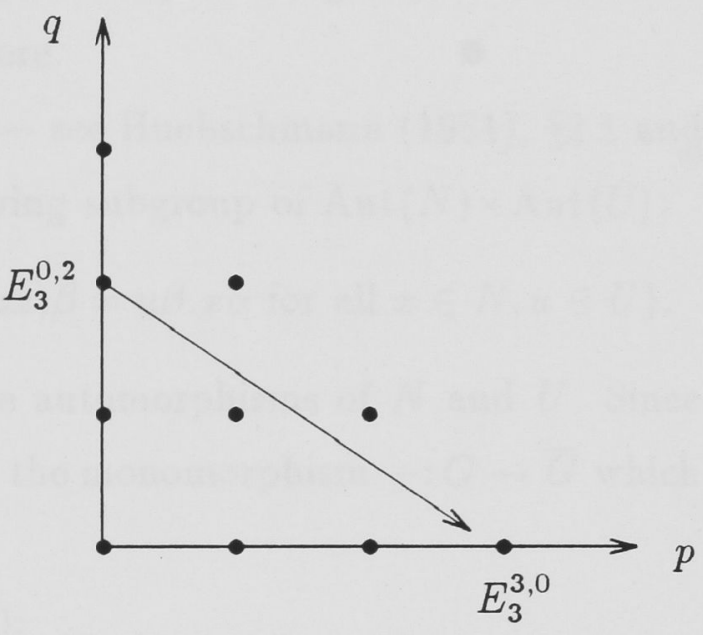

$E_{3}$

Figure 3.1: LHS spectral sequence differentials of total degree at most 2

A useful consequence of (3.10) is the following.

Proposition 3.2.4 If $G$ and $U$ are finite and $H^{i}(N, U)=0$ for $1 \leq i \leq 2$, then

$$
\left|H^{2}(G, U)\right|=\left|H^{2}\left(G / N, U^{N}\right)\right|
$$

Remark 3.2.5 It is not always necessary to stipulate that $H^{2}(N, U)=0$ in Proposition 3.2.4. For example, if either $N$ is cyclic, or $N$ and $U$ are $p$-groups, then $H^{1}(N, U)=0$ implies that $H^{i}(N, U)=0$ for all $i \geq 1$ (see Remark 3.1.9 in the first case, and Gruenberg (1970), p.110, Lemma 2 in the second). 


\subsection{Differentials in the LHS spectral sequence}

Let $G$ be a group with normal subgroup $N$ and $U$ a $G$-module. In this section, we determine the action of the LHS spectral sequence differentials $d_{2}^{0,2}, d_{3}^{0,2}$ and $d_{2}^{1,1}$ in various group-theoretic contexts. The discussion is based on the more general treatment given in Huebschmann (1981). It is convenient to assume here that $U$ is faithful as $G$-module (when $|T|=4$, this is because of Theorem 2.3.5; an easy argument [which we do not state here] then shows that it is enough to extend only the faithful finite $D$-submodules of $B$ ). The exposition in Huebschmann (1981), for general $U$, is more complicated.

The differential $d_{2}^{0,1}$ may be described group-theoretically (this description was shown to me by Dr L. G. Kovács). However, in the applications in Section 3.4, we use a description of $d_{2}^{0,1}$ which is derived independently of the group-theoretic one, and so we do not give the latter description here.

We begin with a well-known construction - see Huebschmann (1981), §2.1 and Robinson (1981), §4, p.66. Consider the following subgroup of $\operatorname{Aut}(N) \times \operatorname{Aut}(U)$ :

$$
\operatorname{Aut}(N, U)=\{(\alpha, \beta) \in \operatorname{Aut}(N) \times \operatorname{Aut}(U) \mid(u x) \beta=u \beta . x \alpha \text { for all } x \in N, u \in U\}
$$

The elements of $\operatorname{Aut}(N, U)$ are the compatible automorphisms of $N$ and $U$. Since $U$ is faithful, $G$ is embedded in $\operatorname{Aut}(N, U)$ via the monomorphism $-: G \rightarrow \bar{G}$ which maps $g \in G$ to $\bar{g} \in \bar{G}$, where

$$
\bar{g}=(\tilde{g}, g \chi)
$$

$\tilde{g} \in \operatorname{Aut}(N)$ denotes conjugation by $g$ in $N$ and $\chi$ is the (injective) coupling of $G$ to $U$ prescribing the action of $G$ on $U$. Let

$$
0 \rightarrow U \stackrel{\iota}{\rightarrow} E \stackrel{\pi}{\rightarrow} N \rightarrow 1
$$

be an extension of $U$ by $N$ whose coupling is given by the action of $N$ on $U$. As in the discussion before Proposition 3.1.4, $\theta \in N_{\mathrm{Aut}(E)}(U)$ induces automorphisms $\theta_{N}$ of $N$ and $\theta_{U}$ of $U$, dependent on the extension (3.11) under consideration. By Proposition 3.1.4, $\theta_{N}$ and $\theta_{U}$ are compatible. Then it is easy to check that

$$
\gamma: \theta \mapsto\left(\theta_{N}, \theta_{U}\right)
$$

defines a homomorphism of $N_{\operatorname{Aut}(E)}(U)$ into Aut $(N, U)$. By Proposition 3.1.5, ker $\gamma$ may be identified with $\operatorname{Der}(N, U)$ and this yields an exact sequence

$$
0 \rightarrow \operatorname{Der}(N, U) \rightarrow N_{\operatorname{Aut}(E)}(U) \stackrel{\gamma}{\rightarrow} \operatorname{Aut}(N, U),
$$


where the leftmost nonzero homomorphism maps $\delta \in \operatorname{Der}(N, U)$ to $\alpha_{\delta} \in N_{\operatorname{Aut}(E)}(U)$, as defined in the proof of Proposition 3.1.5. Denote by $\operatorname{Aut}_{G}(E)$ the complete inverse image of $\operatorname{im} \gamma \cap \bar{G}$ in $N_{\operatorname{Aut}(E)}(U)$ under $\gamma$. Proposition 3.1 .4 immediately implies the next result.

Proposition 3.3.1 $\operatorname{Aut}_{G}(E)$ is mapped epimorphically onto $\bar{G}$ by $\gamma$ if and only if the equivalence class of the extension (3.11) is an element of $H^{2}(N, U)^{G / N}$.

Assume now that the equivalence class of the extension (3.11) is $G$-fixed, so that $\gamma$ maps $\operatorname{Aut}_{G}(E)$ onto $\bar{G}$. Denote the canonical homomorphisms $E \rightarrow \operatorname{Inn}(E)$ and $U \rightarrow \operatorname{Ider}(N, U)$ by $\mu$ and $\nu$ respectively; note that $E \mu \leq \operatorname{Aut}_{G}(E)$. By Proposition 3.1 .5 we have the following commutative diagram:

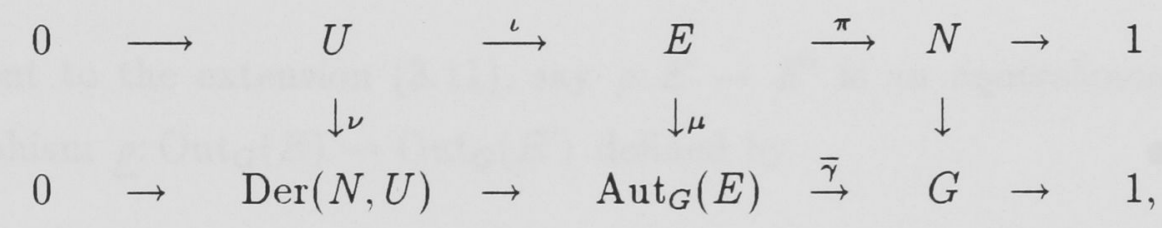

where the rightmost vertical map is inclusion and $\bar{\gamma}$ is the composite of $\gamma$ as defined above with the inverse of the embedding - of $G$ in $\operatorname{Aut}(N, U)$. Passing to cokernels, and denoting the subgroup $\operatorname{Aut}_{G}(E) / \operatorname{Inn}(E)$ of $\operatorname{Out}(E)$ by $\operatorname{Out}_{G}(E)$, we obtain the short exact sequence

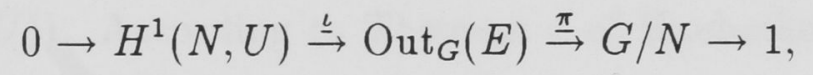

where $\underline{\iota}$ is defined by

$$
\underline{\iota}: \delta+\operatorname{Ider}(N, U) \mapsto \alpha_{\delta} \operatorname{Inn}(E)
$$

for $\delta \in \operatorname{Der}(N, U)$, and $\underline{\pi}$ by

$$
\underline{\pi}: \theta \operatorname{Inn}(E) \mapsto \theta \bar{\gamma} N
$$

for $\theta \in \operatorname{Aut}_{G}(E)$.

Lemma 3.3.2 The equivalence class of $(3.12)$ is an element of $H^{2}\left(G / N, H^{1}(N, U)\right)$.

Proof. We have to show that the coupling of the extension (3.12) is the same as that given by the action of $G / N$ on $H^{1}(N, U)$. For $g \in G$, choose $\theta \in N_{\text {Aut (E) }}(U)$ such that $(\theta \operatorname{Inn}(E)) \underline{\pi}=g N$. By Proposition 3.1.4, if $x \in N$ then for $(x, u) \in E$ there is a 1-cochain $\phi$ from $N$ to $U$ such that

$$
(x, u) \theta=\left(x^{g}, u g+x^{g} \phi\right) .
$$


If $\delta \in \operatorname{Der}(N, U)$ then $\alpha_{\delta} \theta \operatorname{Inn}(E)$ is mapped to $\delta_{\theta-1} \alpha_{\delta} \theta+\operatorname{Ider}(N, U)$ under $\underline{\iota}^{-1}$. Using the definition of $\delta_{\theta-1} \alpha_{\delta} \theta$ given in the proof of Proposition 3.1.5, we see that

$$
\begin{aligned}
x \delta_{\theta^{-1} \alpha_{\delta} \theta} & =(x, u)^{-1} \cdot(x, u) \theta^{-1} \alpha_{\delta} \theta \\
& =(x, u)^{-1} \cdot\left(x^{g^{-1}}, u g^{-1}-x \phi \cdot g^{-1}\right) \alpha_{\delta} \theta \\
& =(x, u)^{-1}\left(\left(x^{g^{-1}}, u g^{-1}-x \phi \cdot g^{-1}\right)\left(1, x^{g^{-1}} \delta\right)\right) \theta \\
& =(x, u)^{-1}(x, u)\left(1, x^{g^{-1}} \delta \cdot g\right) \\
& =\left(1, x^{g^{-1}} \delta . g\right) .
\end{aligned}
$$

Thus $\delta_{\theta^{-1} \alpha_{\delta} \theta}=\delta \circ g N$, proving the lemma.

Suppose that

$$
0 \rightarrow U \stackrel{\iota^{\prime}}{\rightarrow} E^{\prime} \stackrel{\pi^{\prime}}{\rightarrow} N \rightarrow 1
$$

is equivalent to the extension (3.11), say $\rho: E \rightarrow E^{\prime}$ is an equivalence. Then the homomorphism $\underline{\rho}: \operatorname{Out}_{G}(E) \rightarrow \operatorname{Out}_{G}\left(E^{\prime}\right)$ defined by

$$
\theta \operatorname{Inn}(E) \underline{\rho}=\theta^{\rho} \operatorname{Inn}\left(E^{\prime}\right)
$$

is likewise an equivalence of extensions. Together with Lemma 3.3.2, this shows that the map $d_{2}^{0,2}$ sending the ( $G$-fixed) equivalence class of the extension (3.11) to the equivalence class of the extension $(3.12)$ is a well-defined map from $H^{2}(N, U)^{G / N}$ to $H^{2}\left(G / N, H^{1}(N, U)\right)$. According to Theorem 1 of Huebschmann (1981), $d_{2}^{0,2}$ so defined is the relevant LHS spectral sequence differential.

The next differential we consider is $d_{2}^{1,1}: H^{1}\left(G / N, H^{1}(N, U)\right) \rightarrow H^{3}\left(G / N, U^{N}\right)$. The image under $d_{2}^{0,2}$ of the equivalence class of the split extension $E=U \rtimes N$ is the equivalence class of the extension

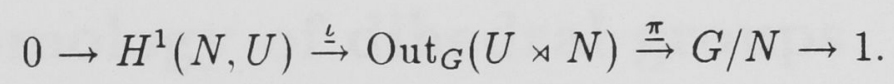

In fact, the extension (3.13) splits over $H^{1}(N, U)$. To see this, consider the transversal function $\sigma$ from $G / N$ into $\mathrm{Out}_{G}(U \rtimes N)$ which is (well-)defined for $g \in G$ by

$$
g N \sigma=g \operatorname{Inn}(U \rtimes N),
$$

where $g$ in the right-hand side of this definition denotes the element of $\operatorname{Aut}_{G}(U \rtimes N)$ defined for $x \in N$ and $u \in U$ by

$$
(x, u) g=\left(x^{g}, u g\right) .
$$


It is straightforward to verify that $\sigma$ is a homomorphism. (Of course, the existence of a transversal homomorphism is guaranteed by Huebschmann's proof that $d_{2}^{0,2}$ is a homomorphism.) For each derivation $\delta$ from $G / N$ to $H^{1}(N, U)$, define the map $\sigma_{\delta}: G / N \rightarrow \mathrm{Out}_{G}(U \rtimes N)$ by

$$
g N \sigma_{\delta}=g N \sigma \cdot g N \delta \underline{\imath}
$$

Now $\sigma_{\delta}$ is a homomorphism (recall the discussion after Theorem 3.1.3). Thus $\sigma_{\delta}$, composed with the inclusion of $\operatorname{Out}_{G}(U \rtimes N)$ in $\operatorname{Out}(U \rtimes N)$, is a coupling of $G / N$ to $U \rtimes N$. Since $\zeta(U \rtimes N)=U^{N}$ by the assumption that $U$ is faithful, the cohomology class of an obstruction $\kappa_{\delta}$ determined by $\sigma_{\delta}$ is an element of $H^{3}\left(G / N, U^{N}\right)$. This cohomology class is determined by the cohomology class of $\delta$. According to Theorem 2 of Huebschmann (1981), $d_{2}^{1,1}$ maps $[\delta]$ to $\left[\kappa_{\delta}\right]$.

Finally we come to $d_{3}^{0,2}$. In view of the discussion in $\S 10$ of Huebschmann (1980), the relevant special case of Theorem 3 of Huebschmann (1981) may be paraphrased as follows. When the equivalence class of the extension (3.11) lies in the domain ker $d_{2}^{0,2}$ of $d_{3}^{0,2}$, there is a corresponding split extension (3.12). Choose a transversal homomorphism $\sigma: G / N \rightarrow \operatorname{Out}_{G}(E)$. By composing $\sigma$ with the inclusion of $\operatorname{Out}_{G}(E)$ in $\operatorname{Out}(E)$, we obtain a coupling of $G / N$ to $E$. Since $\zeta(E)=U^{N}$, the cohomology class $\left[\kappa_{\delta}\right]$ of an obstruction $\kappa_{\sigma}$ determined by this coupling is an element of $H^{3}\left(G / N, U^{N}\right)$. For fixed $\sigma$, the class $\left[\kappa_{\sigma}\right]$ is determined by the equivalence class of (3.11). As $\sigma$ ranges over the homomorphisms that split (3.12), the $\left[\kappa_{\sigma}\right]$ form a coset modulo im $d_{2}^{1,1}$, that is, an element of the factor group $E_{3}^{3,0}$ [compare (3.14) with (3.5)]. This element is the image under $d_{3}^{0,2}$ of the equivalence class of (3.11).

\subsection{Cohomology of dihedral groups}

In this section, we consider the special case that $G$ is $D_{2 n}$, the dihedral group of order $2 n, n \geq 2$ :

$$
G=\left\langle x, y \mid x^{n}=y^{2}=1, x^{y}=x^{-1}\right\rangle
$$

Set $N=\langle x\rangle \triangleleft G$. The subsequent discussion specialises to $G=V_{4}$ and $G=D$. We assume that the $G$-module $U$ is faithful (not all results require this).

Both $N$ and $G / N$ are finite cyclic, so that the cohomology of these groups with coefficients in $U$ may be expressed in terms of sections of $U$. We will determine the actions of $d_{2}^{0,1}$ and the LHS spectral sequence differentials discussed in Section 3.3 
on these sections of $U$. Thus, we need to understand the action of $G$ on these sections which corresponds to the action induced by (3.6) under the isomorphisms of Proposition 3.1.8. As we commented in Remark 3.2.1, this is not necessarily the same action as that induced by the given action of $G$ on $U$. Since the action of $N$ on its own cohomology groups is trivial, only the action of $y$ needs to be given. This is done first for $(2 m-1)$-cohomology and then $2 m$-cohomology, $m \geq 1$.

For an endomorphism $f$ of $U$ and a submodule $U^{\prime}$, the complete inverse image $\left\{u \in U \mid u f \in U^{\prime}\right\}$ is denoted by $U^{\prime} f^{-1}$. We denote cosets in $U$, and also cohomology class, by $[-]$.

Lemma 3.4.1 Define an action o of $G$ on $\operatorname{ker}\left(1+x+\cdots+x^{n-1}\right) / U(1-x)$ by

$$
[u] \circ y=-[u y x]
$$

for $u \in \operatorname{ker}\left(1+x+\cdots+x^{n-1}\right)$. With this action, the isomorphism

$$
\bar{\alpha}: H^{1}(N, U) \rightarrow \operatorname{ker}\left(1+x+\cdots+x^{n-1}\right) / U(1-x)
$$

induced by $\alpha$ as defined in Remark 3.1 .10 becomes a $G$-isomorphism. Under $\bar{\alpha}$, $H^{1}(N, U)^{G / N}$ is mapped onto

$$
\left(\operatorname{ker}\left(1+x+\cdots+x^{n-1}\right) \cap U(1-x)(1+y x)^{-1}\right) / U(1-x)
$$

Proof. By Remark 3.1.10, $\bar{\alpha}$ is defined by

$$
\bar{\alpha}: \delta+\operatorname{Ider}(N, U) \mapsto x \delta+U(1-x)
$$

for $\delta \in \operatorname{Der}(N, U)$. The action of $y$ induced by (3.6) is given by

$$
(\delta+\operatorname{Ider}(N, U)) \circ y=\delta \circ y+\operatorname{Ider}(N, U)
$$

where $z(\delta \circ y)=z^{-1} \delta y$ for $z \in N$. Since $x^{-1} \delta=-x \delta x^{-1}$ and $x^{-1} y=y x$, the first assertion follows. The second assertion is a trivial consequence.

Remark 3.4.2 An equivalent definition of $\circ$ is $(u+U(1-x)) \circ y=-u y+U(1-x)$. We use the definition given in Lemma 3.4.1 because it makes later calculations easier.

Lemma 3.4.3 Assume $U$ finite. Define an action of $\langle y\rangle$ on

$$
\operatorname{ker}(1-x) / U\left(1+x+\cdots+x^{n-1}\right)
$$


by setting

$$
[u] \circ y=-[u y]
$$

for $u \in \operatorname{ker}(1-x)$. With respect to this action, $a\langle y\rangle$-isomorphism of $H^{2}(N, U)$ onto $\operatorname{ker}(1-x) / U\left(1+x+\cdots+x^{n-1}\right)$ may be defined by

$$
[\psi] \mapsto \sum_{i=1}^{n-1}\left(x, x^{i}\right) \psi+U\left(1+x+\cdots+x^{n-1}\right)
$$

for each 2-cocycle $\psi$ from $N$ to $U$.

Proof. Obviously, $\psi \mapsto \sum_{i=1}^{n-1}\left(x, x^{i}\right) \psi$ is a homomorphism. Its image lies in $\operatorname{ker}(1-x)$ : for $\psi d_{3}^{*}=0$, and, by $(3.1), \sum_{i=1}^{n-1}\left(x, x^{i}, x\right) \psi d_{3}^{*}=\left(\sum_{i=1}^{n-1}\left(x, x^{i}\right) \psi\right)(1-x)$. Its image is precisely $\operatorname{ker}(1-x)$ : for, if $u \in \operatorname{ker}(1-x)$, then a normalised 2-cochain $\psi_{u}$ from $N$ to $U$ may be defined by

$$
\left(x^{i}, x^{j}\right) \psi_{u}= \begin{cases}0 & \text { if } i+j<n \\ u & \text { if } i+j \geq n\end{cases}
$$

$0 \leq i, j<n$. Using (3.1), we may verify that $\psi_{u} d_{3}^{*}=0$. Also, $\sum\left(x, x^{i}\right) \psi_{u}=u$. If $\phi$ is a 1-cochain from $N$ to $U$, then

$$
\begin{aligned}
\sum\left(x, x^{i}\right) \phi d_{2}^{*} & =\sum\left(x^{i} \phi-x^{i+1} \phi+x \phi x^{i}\right) \\
& =x \phi\left(1+x+\cdots+x^{n-1}\right) \\
& \in U\left(1+x+\cdots+x^{n-1}\right),
\end{aligned}
$$

so that the original homomorphism induces a surjective homomorphism

$$
[\psi] \mapsto \sum_{i=1}^{n-1}\left(x, x^{i}\right) \psi+U\left(1+x+\cdots+x^{n-1}\right)
$$

of $H^{2}(N, U)$ onto $\operatorname{ker}(1-x) / U\left(1+x+\cdots+x^{n-1}\right)$. By Proposition 3.1.8, these two groups are isomorphic, so that this surjective homomorphism must be an isomorphism. By definition, $\sum_{i=1}^{n-1}\left(x, x^{i}\right)\left(\psi_{u} \circ y\right)=\sum_{i=1}^{n-1}\left(x^{n-1}, x^{n-i}\right) \psi_{u} y=(n-1) u y$. Since $u \in \operatorname{ker}(1-x)$, we have $n u y=u\left(1+x+\cdots+x^{n-1}\right) y=u y\left(1+x+\cdots+x^{n-1}\right)$. Therefore, $(n-1) u y \in-u y+U\left(1+x+\cdots+x^{n-1}\right)$ and $\left[\psi_{u} \circ y\right]$ is mapped to $-[u y]$, that is, to $[u] \circ y$, as required.

The next result is needed in the proof of Theorem 3.4.5.

Lemma 3.4.4 Suppose that $\delta_{1} \in \operatorname{Der}(N, U), \delta_{2} \in \operatorname{Der}(\langle y\rangle, U)$ and

$$
x \delta_{1}(1+y x)=y \delta_{2}(1-x)
$$


Then the map $\delta: G \rightarrow U$ defined by $\left(x^{i}\right) \delta=\left(x^{i}\right) \delta_{1}$ and

$$
\left(y x^{i}\right) \delta=y \delta_{2} x^{i}+x^{i} \delta_{1}
$$

for $1 \leq i \leq n-1$, is a derivation.

Proof. By (3.15),

$$
\begin{aligned}
x^{i} \delta_{1}\left(1+y x^{i}\right) & =x \delta_{1}\left(1+x+\cdots+x^{i-1}\right)\left(1+y x^{i}\right) \\
& =x \delta_{1}(1+y x)\left(1+x+\cdots+x^{i-1}\right) \\
& =y \delta_{2}(1-x)\left(1+x+\cdots+x^{i-1}\right) \\
& =y \delta_{2}\left(1-x^{i}\right)
\end{aligned}
$$

for $i \geq 1$. Therefore, since $x^{i} \delta_{1} x^{-i}=-x^{-i} \delta_{1}\left(\delta_{1}\right.$ is a derivation from $N$ to $\left.U\right)$,

$$
y \delta_{2} x^{i}+x^{i} \delta_{1}=x^{-i} \delta_{1} y+y \delta_{2}
$$

To see that (3.16) defines a derivation from $G$ to $U$, we must verify that

$$
\left(y^{i} x^{j} y^{k} x^{l}\right) \delta=\left(y^{i} x^{j}\right) \delta y^{k} x^{l}+\left(y^{k} x^{l}\right) \delta
$$

for $0 \leq i, k \leq 1,0 \leq j, l \leq n-1$. For instance, using first (3.17) with $-i$ in place of $i$ and then the fact that $y \delta_{2}=-y \delta_{2} y$, we have

$$
\begin{aligned}
\left(y x^{i}\right) \delta y x^{j}+\left(y x^{j}\right) \delta & =y \delta_{2} x^{i} y x^{j}+x^{i} \delta_{1} y x^{j}+y \delta_{2} x^{j}+x^{j} \delta_{1} \\
& =\left(y \delta_{2} x^{i} y+x^{i} \delta_{1} y+y \delta_{2}\right) x^{j}+x^{j} \delta_{1} \\
& =\left(y \delta_{2} x^{i} y+y \delta_{2} x^{-i}+x^{-i} \delta_{1}\right) x^{j}+x^{j} \delta_{1} \\
& =x^{-i} \delta_{1} x^{j}+x^{j} \delta_{1} \\
& =\left(y x^{i} \cdot y x^{j}\right) \delta .
\end{aligned}
$$

The other verifications are equally straightforward.

Now we move on to the descriptions of $d_{2}^{0,1}$ and the LHS spectral sequence differentials discussed in Section 3.3.

Recall that the domain $H^{1}(N, U)^{G / N}$ and codomain $H^{2}\left(G / N, U^{N}\right)$ of $d_{2}^{0,1}$ may be identified, by Lemma 3.4.1 and Proposition 3.1.8, as

$$
\left(\operatorname{ker}\left(1+x+\cdots+x^{n-1}\right) \cap U(1-x)(1+y x)^{-1}\right) / U(1-x)
$$

and

$$
U^{G} / U^{N}(1+y)
$$


respectively. We define below a homomorphism $\tau$ from the first of these sections of $U$ to the second, which (with those identifications) is probably the same as $d_{2}^{0,1}$. However, we only intend to use $\tau$ for computing the order of ker $d_{2}^{0,1}$ in specific cases, so all we need to prove is that $|\operatorname{ker} \tau|=\left|\operatorname{ker} d_{2}^{0,1}\right|(\operatorname{ker} \tau$ is finite if and only if $\operatorname{ker} d_{2}^{0,1}$ is finite, and in that case the two kernels have the same order).

Theorem 3.4.5 For $u \in \operatorname{ker}\left(1+x+\cdots+x^{n-1}\right) \cap U(1-x)(1+y x)^{-1}$, choose $v \in U$ such that

$$
v(1-x)=u(1+y x)
$$

and set

$$
\tau: u+U(1-x) \mapsto v(1+y)+U^{N}(1+y)
$$

This defines a homomorphism

$$
\tau:\left(\operatorname{ker}\left(1+x+\cdots+x^{n-1}\right) \cap U(1-x)(1+y x)^{-1}\right) / U(1-x) \rightarrow U^{G} / U^{N}(1+y)
$$

and $|\operatorname{ker} \tau|=\left|\operatorname{ker} d_{2}^{0,1}\right|$.

Proof. The following identities in $\mathbb{Z} G$ will be useful:

$$
(1-x)(1+y x)=(1-y)(1-x)
$$

and

$$
(1-x)(1-y x)=(1+y)(1-x)
$$

The first thing to do is prove that $\tau$ is well-defined. This involves three separate verifications. First, $v(1+y)+U^{N}(1+y)$ does not depend on the choice of $v$. For if $v^{\prime}(1-x)=v(1-x)$ then $v-v^{\prime} \in U^{N}$ and so $v^{\prime}(1+y) \equiv v(1+y) \bmod U^{N}(1+y)$. Second, if $u$ is replaced by another representative $u+w(1-x)$ of its coset, then by (3.18) we have

$$
\begin{aligned}
(u+w(1-x))(1+y x) & =u(1+y x)+w(1-x)(1+y x) \\
& =(v+w(1-y))(1-x) .
\end{aligned}
$$

Since $(1-y)(1+y)=0$, this shows that the image of $u+w(1-x)+U(1-x)$ under $\tau$ is the same as that of $u+U(1-x)$. Third, by (3.19),

$$
\begin{aligned}
v(1+y)(1-x) & =v(1-x)(1-y x) \\
& =u(1+y x)(1-y x) \\
& =0
\end{aligned}
$$


Since it is certainly true that $v(1+y) \in \operatorname{ker}(1-y)$, we see that the images do indeed lie in $U^{G} / U^{N}(1+y)$.

Additivity of $\tau$ is obvious. Recall from (3.9) that $\operatorname{ker} d_{2}^{0,1}=$ im res. To complete the proof, we show that $\operatorname{ker} \tau$ corresponds to im res under the isomorphism $\bar{\alpha}$ defined in Lemma 3.4.1.

Suppose that $\delta \in \operatorname{Der}(G, U)$. Then $[\delta]$ res $=\left.\delta\right|_{N}+\operatorname{Ider}(N, U)$ and $\bar{\alpha}$ maps this element of $H^{1}(N, U)^{G / N}$ to $x \delta+U(1-x)$. Since $\delta$ is a derivation from $G$ to $U$, it follows that

$$
y \delta(1+y)=0
$$

and $y \delta=(x y x) \delta=x \delta(1+y x)+y \delta x ;$ that is

$$
y \delta(1-x)=x \delta(1+y x)
$$

By definition, $\tau$ therefore maps $x \delta+U(1-x)$ to $y \delta(1+y)+U^{N}(1+y)=0$. Hence (im res) $\bar{\alpha} \leq \operatorname{ker} \tau$. Conversely, suppose that $u+U(1-x) \in \operatorname{ker} \tau$, that is, $v(1+y)=z(1+y)$ for some $z \in U^{N}$. Set $w=v-z$, so that $w(1-x)=u(1+y x)$ and $w \in \operatorname{ker}(1+y)$. Set $u \alpha=\delta_{1} \in \operatorname{Der}(N, U)$ and define $\delta_{2} \in \operatorname{Der}(\langle y\rangle, U)$ by $y \delta_{2}=w$. By Lemma 3.4.4, we obtain a derivation $\delta$ from $G$ to $U$ whose restriction to $N$ is $\delta_{1}$. Therefore, $\operatorname{ker} \tau \leq(\mathrm{im}$ res $) \bar{\alpha}$ and we are done.

Remark 3.4.6 For the $G$-modules $U$ that we consider in Chapter 5 (and the analogous modules for $T=D), N$ is chosen so that $H^{2}\left(G / N, U^{N}\right)$ is either zero or a $C_{2}$. Then $d_{2}^{0,1}$, determined completely by (the order of) its kernel, matches $\tau$ as defined in Theorem 3.4.5.

We do not need a full description of $d_{2}^{0,2}$; rather, we need to be able to calculate $\operatorname{ker} d_{2}^{0,2}=E_{3}^{0,2}$.

Proposition 3.4.7 Let $\psi$ be a 2 -cocycle from $N$ to $U$ such that $[\psi] \in H^{2}(N, U)^{G / N}$. Then $[\psi] d_{2}^{0,2}=0$ if and only if for some 1 -cochain $\phi$ from $N$ to $U$ satisfying

$$
\psi \circ y=\psi+\phi d_{2}^{*}
$$

there is $v \in U$ such that for all $z \in N$,

$$
z^{-1} \phi y+z \phi=v(1-z)
$$


Proof. Let (3.11) be an extension of $U$ by $N$ giving rise to $\psi$. By definition, $[\psi] d_{2}^{0,2}=0$ if and only if the extension (3.12) splits, that is, some element of $y \underline{\pi}^{-1}$ has trivial square. By Proposition 3.1.4, each element of $y \underline{\pi}^{-1}$ is of the form $\theta_{\phi} \operatorname{Inn}(E)$ modulo $H^{1}(N, U) \underline{\iota}$, where $\phi$ is a 1 -cochain from $N$ to $U$ satisfying $(3.20)$ [the condition that $[\psi]$ is $G$-fixed], and $\theta_{\phi} \in \operatorname{Aut}_{G}(E)$ is defined by

$$
(z, u) \theta_{\phi}=\left(z^{-1}, u y+z^{-1} \phi\right)
$$

for $(z, u) \in E$. An element of $H^{1}(N, U) \underline{\iota}$ is of the form $\alpha_{\delta} \operatorname{Inn}(E)$ for some $\delta \in \operatorname{Der}(N, U)$. By definition, $\theta_{\phi} \alpha_{\delta}=\theta_{\phi+\delta}$ and certainly $\phi+\delta$ satisfies $(3.20)$ if and only if $\phi$ does. Hence (3.12) splits if and only if $\theta_{\phi}^{2} \in \operatorname{Inn}(E)$ for some such $\theta_{\phi}$. That is, there is a fixed element $\left(z^{\prime}, v\right) \in E$ such that

$$
\begin{aligned}
\left(z, u+z^{-1} \phi y+z \phi\right) & =(z, u)^{\left(z^{\prime}, v\right)} \\
& =\left(z, u z^{\prime}+v(1-z)+\left(z, z^{\prime}\right) \psi-\left(z^{\prime}, z\right) \psi\right)
\end{aligned}
$$

for all $(z, u) \in E$, where we have used the associativity identity (3.3) for 2-cocycles. In particular, for $z=1$, the faithfulness of $U$ implies that $z^{\prime}=1$. This completes the proof.

The next two results deal with some special cases of Proposition 3.4.7. The first follows from the fact that $d_{2}^{0,2}$ is a homomorphism, but is easily verified independently (as we do).

Lemma 3.4.8 Let $\psi$ be a 2-cocycle from $N$ to $U$ such that $[\psi] \in H^{2}(N, U)^{G / N}$, and choose a 1 -cochain $\phi$ from $N$ to $U$ satisfying (3.20). If there is some $v \in U$ such that

$$
x^{-1} \phi y+x \phi=v(1-x)
$$

then $[\psi] d_{2}^{0,2}=0$

Proof. We prove that $x^{-i} \phi y+x^{i} \phi=v\left(1-x^{i}\right)$, by induction on $i$. This implies the result, by Proposition 3.4.7.

Expanding $(3.20)$ by $(3.1)$, first for $\left(x^{i}, x\right)$ and then for $\left(x^{-i}, x^{-1}\right)$, we have

$$
\left(x^{-i}, x^{-1}\right) \psi y=\left(x^{i}, x\right) \psi+x^{i} \phi x-x^{i+1} \phi+x \phi
$$

and

$$
\left(x^{i}, x\right) \psi=\left(x^{-i}, x^{-1}\right) \psi y+x^{-i} \phi y x-x^{-(i+1)} \phi y+x^{-1} \phi y
$$


Therefore,

$$
x^{-i} \phi y x+x^{i} \phi x+x^{-1} \phi y+x \phi=x^{-(i+1)} \phi y+x^{i+1} \phi
$$

and

$$
\begin{aligned}
v\left(1-x^{i+1}\right) & =v\left(1-x^{i}\right) x+v(1-x) \\
& =x^{-i} \phi y x+x^{i} \phi x+x^{-1} \phi y+x \phi \\
& =x^{-(i+1)} \phi y+x^{i+1} \phi
\end{aligned}
$$

by induction.

Lemma 3.4.9 Let $\psi$ be a 2-cocycle from $N$ to $U$ such that $[\psi] \in H^{2}(N, U)^{G / N}$ and suppose that $\psi$ has values in $U^{G}$. If there is a 1 -cochain $\phi$ from $N$ to $U$ with values in $U^{G}$ and satisfying (3.20), then $[\psi] d_{2}^{0,2}=0$.

Proof. Since $\psi$ is a 2 -cocycle with values in $U^{G},\left(x, x^{-1}\right) \psi=\left(x^{-1}, x\right) \psi$ by $(3.3)$. Then by $(3.20)$,

$$
\left(x, x^{-1}\right) \psi=\left(x^{-1}, x\right) \psi+x^{-1} \phi x+x \phi,
$$

that is, $x^{-1} \phi y+x \phi=0$. Therefore, we may choose $v=0$ in Lemma 3.4.8.

Proposition 3.4.7 and Lemmas 3.4.8 and 3.4.9 enable us to determine $\operatorname{ker} d_{2}^{0,2}$ for all $G$-modules that will be considered in Chapter 5 (and those for $T=D$ ).

Next, we consider $d_{2}^{1,1}$. Recall the discussion before Theorem 3.1 .6 and the description of $d_{2}^{1,1}$ given in Section 3.3. Choose $\delta \in \operatorname{Der}\left(G / N, H^{1}(N, U)\right)$ and a transversal homomorphism $\sigma: G / N \rightarrow \mathrm{Out}_{G}(U \rtimes N)$, and define a coupling $\sigma_{\delta}$ from $G / N$ to $U \rtimes N$ by (3.14). Choose $d_{y} \in \operatorname{Der}(N, U)$ such that $y \delta=d_{y}+\operatorname{Ider}(N, U)$. Explicitly, $\sigma_{\delta}$ acts on $G / N$ by

$$
y \sigma_{\delta}=y \alpha_{d_{y}} \operatorname{Inn}(U \rtimes N)
$$

where $y \in \operatorname{Aut}_{G}(U \rtimes N)$ is defined by

$$
(z, u) y=\left(z^{-1}, u y\right)
$$

for $z \in N, u \in U$, and $\alpha_{d_{y}}$ is defined as in the proof of Proposition 3.1.5. Set $y \lambda=y \alpha_{d_{y}}$ and $1 \lambda=1$. We wish to choose an element $(y, y) \psi \in U \rtimes N$ which induces the inner automorphism $(y \lambda)^{2}$ of $U \rtimes N$. Now $\delta$ is a derivation and so $y \delta y+y \delta=0$, that is, $d_{y} \circ y+d_{y} \in \operatorname{Ider}(N, U)$. Together with $z^{-1} d_{y}=-z d_{y} z^{-1}$, this implies that there is some $v \in U$ such that

$$
z d_{y} y z=z d_{y}-v(1-z)
$$


for all $z \in N$. By definition, $(z, u) y \alpha_{d_{y}}=\left(z^{-1}, u y+z^{-1} d_{y}\right)$, and so by (3.22),

$$
(z, u)\left(y \alpha_{d_{y}}\right)^{2}=(z, u)^{(1, v)}
$$

Therefore, we may choose $(y, y) \psi=(1, v)$. An obstruction $\kappa_{\delta}$ determined by the coupling $\sigma_{\delta}$ is defined by

$$
(1, v)=(1, v) y \alpha_{d_{y}}+(y, y, y) \kappa_{\delta}
$$

that is,

$$
(y, y, y) \kappa_{\delta}=v(1-y)
$$

By an inductive method similar to that used in the proof of Lemma 3.4.8, it may be verified that (3.22) is satisfied by all $z \in N$ if and only if it is satisfied by $x$. Also,

$$
[\psi] \mapsto[(y, \ldots, y) \psi]
$$

clearly defines an isomorphism of $H^{i}\left(\langle y\rangle, U^{N}\right)$ onto the relevant section of $U$. Using these facts and Lemma 3.4.1, we reformulate the preceding discussion as follows.

Proposition 3.4.10 For $u \in \operatorname{ker}\left(1+x+\cdots+x^{n-1}\right) \cap U(1-x)(1-y x)^{-1}$, choose $v \in U$ such that

$$
u(1-y x)=v(1-x)
$$

and set

$$
d_{2}^{1,1}: u+\operatorname{ker}\left(1+x+\cdots+x^{n-1}\right)(1+y x) \mapsto v(1-y)+U^{N}(1-y)
$$

This defines a homomorphism

$$
\begin{aligned}
& \left(\operatorname{ker}\left(1+x+\cdots+x^{n-1}\right) \cap U(1-x)(1-y x)^{-1}\right) / \operatorname{ker}\left(1+x+\cdots+x^{n-1}\right)(1+y x) \rightarrow \\
& \left(\operatorname{ker}(1+y) \cap U^{N}\right) / U^{N}(1-y)
\end{aligned}
$$

which corresponds to the LHS spectral sequence differential, denoted by the same notation, under the relevant isomorphisms as defined in Lemma 3.1 .10 and (3.23). In particular, if $v \in U^{G / N}$ then $[u] d_{2}^{1,1}=0$.

By Lemma 3.4.3, when $U$ is finite, $H^{2}(N, U)^{G / N}$ may be identified with

$$
\left(\operatorname{ker}(1-x) \cap\left(1+x+\cdots+x^{n-1}\right)(1+y)^{-1}\right) / U\left(1+x+\cdots+x^{n-1}\right) .
$$

We use this identification in the next proposition. 
Proposition 3.4.11 Assume $U$ finite and let $d_{2}^{1,1}$ be defined as in Proposition 3.4.10. For $u \in \operatorname{ker}(1-x) \cap\left(1+x+\cdots+x^{n-1}\right)(1+y)^{-1}$ such that $[u]$ corresponds to $[\psi] \in \operatorname{ker} d_{2}^{0,2}$ under the isomorphism given in Lemma 3.4.3, choose $\phi$ and $v$ as in Lemma 3.4.8. Then the composite of the (not necessarily homomorphic) map $\tau_{2}$ defined by

$$
\tau_{2}:[u] \mapsto v(1-y)+U^{N}(1-y)
$$

and the natural surjection modulo $\operatorname{im} d_{2}^{1,1}$ corresponds to the LHS spectral sequence differential $d_{3}^{0,2}$. In particular, if $v \in U^{G / N}$ then $[\psi] d_{3}^{0,2}=0$.

Proof. Recall the description of $d_{3}^{0,2}$ given at the end of Section 3.3. As usual, let (3.11) be an extension of $U$ by $N$ and let $\psi$ be a 2 -cocycle arising from this extension. The hypotheses, together with Lemma 3.4.8, imply that $\theta_{\phi}^{2}$ is the inner automorphism of $E$ induced by $v \in U$, where $\theta_{\phi} \in \operatorname{Aut}_{G}(E)$ is defined in the proof of Proposition 3.4.7. We have a transversal homomorphism $\sigma: G / N \rightarrow \operatorname{Out}_{G}(E)$ given by $y \sigma=\theta_{\phi} \operatorname{Inn}(E)$ and hence a coupling of $G / N$ to $E$. We now determine an obstruction $\kappa_{\sigma}$ arising from this coupling. Set $y \lambda=\theta_{\phi}$ and $1 \lambda=1$. Set $(y, y) \psi=(1, v)$, which induces the inner automorphism $(y \lambda)^{2}$ of $E$. Then $\kappa_{\sigma}$ is defined by

$$
(1, v)=(1, v) \theta_{\phi}+(y, y, y) \kappa_{\sigma} .
$$

That is, $(y, y, y) \kappa_{\sigma}=v(1-y)$. The result is now clear. 


\section{Chapter 4}

\section{Submodule lattices of direct sums}

The principal object of study in this chapter is the poset of finite submodules of the direct sum of two modules. This poset is also a lattice. However, we are interested in the inclusion relations between submodules, rather than the lattice operations. A theorem of Remak (1930) gives a description of the set of submodules of the direct sum. Given two submodules described in Remak's terms, it is difficult in general to determine whether one is contained in the other. In the main result of this chapter, Theorem 4.3.5, we show how to determine whether one is contained in the other as maximal submodule. That is, we give a method of describing the edge set of the Hasse diagram of this poset.

\subsection{Background and basic definitions}

In all posets considered, the partial order is denoted $\leq$. A subset of a poset is called convex if $v_{1} \leq x \leq v_{2}$ for any two of its elements $v_{1}$ and $v_{2}$ implies that $x$ is an element of the subset. A subposet is a nonempty subset with the partial order it inherits from the whole poset.

A lattice may be defined either in terms of a partial order, or in terms of two binary operations - meet and join - subject to appropriate axioms. A sublattice is a nonempty subset closed under the meet and join of the whole lattice. A subposet may be a lattice (in terms of its partial order) without being a sublattice. The sublattice $\left\{x \mid v_{1} \leq x \leq v_{2}\right\}$ is convex; such sublattices are called intervals.

The Hasse diagram of a poset is the directed graph whose vertices are the elements of the poset and which has an edge with "top endpoint" $v_{1}$ and "bottom 
endpoint" $v_{2}$ whenever $v_{1}>v_{2}$ and there is no vertex $x$ such that $v_{1}>x>v_{2}$; moreover, these are its only edges. A subgraph is a nonempty subset of the vertex set with all edges of the whole graph between these vertices. We emphasise that the Hasse diagram of a sublattice need not be a subgraph of the Hasse diagram of the whole lattice. Also, not all subgraphs are Hasse diagrams of sublattices; indeed, not even those that are the Hasse diagrams of subposet lattices. In our pictures, edges of the Hasse diagram of the whole poset are drawn as unbroken lines. We indicate that two vertices are comparable by drawing a broken line between them.

It is not clear from the definition that the Hasse diagram of a poset adequately displays the structure of the poset: if two poset elements are comparable, this may not be deducible from the Hasse diagram, since two comparable elements may not be joined by a path of finite length. Indeed, consider the poset $Q$, with the linear ordering of real numbers as the partial order. The Hasse diagram of this poset has no edges. However, for finite posets there is certainly a path of finite length between any two comparable vertices (see Grätzer (1971), p.12, Lemma 1). Also, two finite posets are isomorphic if and only if their Hasse diagrams are isomorphic [see Birkhoff (1935)]

Let $W$ be a module over a ring $R$ with 1 . The poset of finite submodules of $W$, with the inclusion relation on sets as the partial order, is a lattice. This lattice is denoted $\mathcal{L}(W)$. The meet of two elements of $\mathcal{L}(W)$ is their set-theoretic intersection and the join is their module sum. Recall that $\mathcal{L}(W)$ is a modular lattice: for submodules $U_{1}, U_{2}, U_{3}$ of $W$ such that $U_{1} \leq U_{3}$, we have $\left(U_{1}+U_{2}\right) \cap U_{3}=U_{1}+\left(U_{2} \cap U_{3}\right)$. Amongst all sections of $W$, we will be interested only in those which arise as quotients of finite submodules; we call such sections low.

The vertex set of the Hasse diagram of $\mathcal{L}(W)$ is denoted $\mathcal{V}(W)$ and its edge set $\mathcal{E}(W)$. It is clear from the definition of edge that the elements of $\mathcal{E}(W)$ represent composition factors of the module $W$. Accordingly, an element of $\mathcal{E}(W)$ will be denoted by the composition factor that it represents. However, of course, edges in the Hasse diagram of a sublattice of $\mathcal{L}(W)$ need not represent simple modules. Note that between any two comparable vertices of the (possibly infinite) poset $\mathcal{L}(W)$, there is certainly a path of finite length.

From now on, we consider the special case that $W=U \oplus V$, where $U$ and $V$ are $R$-modules such that the Hasse diagrams of $\mathcal{L}(U)$ and $\mathcal{L}(V)$ are known. Of course, in the infinite case, the Hasse diagram of $\mathcal{L}(U \oplus V)$ cannot be drawn literally on a 
piece of paper. But we may not need the whole partial order of $\mathcal{L}(U \oplus V)$ anyway, as is the case, for instance, in inductive proofs on the set of submodules where each inductive step occurs along an edge. Or consider a typical question that arises in our examples: given an element of $\mathcal{V}(U \oplus V)$, which elements of $\mathcal{E}(U \oplus V)$ have that vertex as an endpoint (see Example 5.2.7)? Again, to answer this question we do not need the whole partial order; essentially we only need to know the elements of $\mathcal{E}(U \oplus V)$. Under the right conditions, the elements of $\mathcal{E}(U \oplus V)$ may be read off from certain finite subgraphs of the whole Hasse diagram of $\mathcal{L}(U \oplus V)$. When $\mathcal{L}(U \oplus V)$ is finite, the whole partial order may be read off from the Hasse diagram of $\mathcal{L}(U \oplus V)$; if this Hasse diagram is complicated, the reduction to subgraphs may be a useful and concise way of displaying the partial order. This is the sort of reduction that we aim for, and is more fully explained in Section 4.3.

The methods in this chapter may be applied also to posets of submodules of $U \oplus V$ satisfying other finiteness conditions which ensure that the corresponding Hasse diagrams display the partial order of the poset. Indeed, the proof of Theorem 4.3.5 does not use finiteness of the submodules concerned, and the theorem remains true if we redefine $\mathcal{L}(U \oplus V)$ [and thence $\mathcal{V}(U \oplus V), \mathcal{E}(U \oplus V)$ ] as the lattice of all submodules of $U \oplus V$ and remove "low" from the statement of hypotheses.

An important sublattice of $\mathcal{L}(U \oplus V)$ is

$$
\left\{U_{1} \oplus V_{1} \mid U_{1} \in \mathcal{V}(U), V_{1} \in \mathcal{V}(V)\right\}
$$

this is called the Cartesian product sublattice, and is denoted $\mathcal{L}(U) \times \mathcal{L}(V)$. It is straightforward to see that the edge set of the Hasse diagram of $\mathcal{L}(U) \times \mathcal{L}(V)$ is the union of the following disjoint sets:

$$
\left\{U_{1} \oplus V_{1} / U_{1} \oplus V_{2} \mid U_{1} \in \mathcal{V}(U), V_{1} / V_{2} \in \mathcal{E}(V)\right\}
$$

and

$$
\left\{U_{1} \oplus V_{1} / U_{2} \oplus V_{1} \mid U_{1} / U_{2} \in \mathcal{E}(U), V_{1} \in \mathcal{V}(V)\right\}
$$

Vertices and edges of the Hasse diagram of $\mathcal{L}(U) \times \mathcal{L}(V)$ will be called Cartesian vertices and Cartesian edges respectively. Note that the definition of Cartesian vertices and edges depends on the particular direct decomposition $U \oplus V$ being considered. Cartesian edges represent simple modules and so the Hasse diagram of $\mathcal{L}(U) \times \mathcal{L}(V)$ is a subgraph of the Hasse diagram of $\mathcal{L}(U \oplus V)$. 
The preceding discussion shows that to construct the Cartesian product subgraph, we only need to know the Hasse diagrams of $\mathcal{L}(U)$ and $\mathcal{L}(V)$ - no information about the module structure of either $U$ or $V$ is required. As will be seen, this differs from the construction of the whole Hasse diagram of $\mathcal{L}(U \oplus V)$, where such information is required.

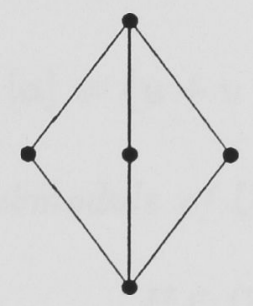

$\mathcal{L}(U)$

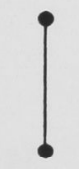

$\mathcal{L}(V)$

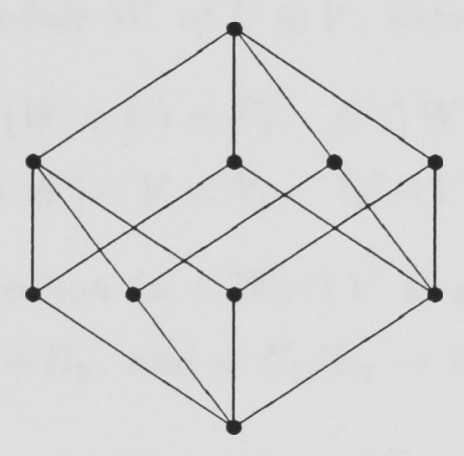

$$
\mathcal{L}(U) \times \mathcal{L}(V)
$$

Figure 4.1: Drawing the Hasse diagram of a Cartesian product

Figure 4.1 provides an example of drawing the Hasse diagram of $\mathcal{L}(U) \times \mathcal{L}(V)$, given the Hasse diagrams of $\mathcal{L}(U)$ and $\mathcal{L}(V)$. In this case, $\mathcal{L}(U)$ is the subspace lattice of $U=V(2,2)$ and $\mathcal{L}(V)$ is the subspace lattice of $V=V(1,2)$. The direct sum $U \oplus V$ is isomorphic to $V(3,2)$. The number of vertices of the Hasse diagram of $\mathcal{L}(U) \times \mathcal{L}(V)$ is $|\mathcal{V}(U)||\mathcal{V}(V)|=10$ and the number of its edges is $|\mathcal{V}(U)||\mathcal{E}(V)|+|\mathcal{E}(U)||\mathcal{V}(V)|=5.1+6.2=17$.

\subsection{The submodules of $U \oplus V$}

Before one can describe the Hasse diagram of $\mathcal{L}(U \oplus V)$, a canonical description of the submodules of $U \oplus V$ is needed. Such a description is realised in Theorem 4.2.1, 
which is usually attributed to Remak (1930). This theorem shows that to list the submodules of $U \oplus V$ it is not enough to know the Hasse diagrams of $\mathcal{L}(U)$ and $\mathcal{L}(V)$ [this much information gives us the Hasse diagram of $\mathcal{L}(U) \times \mathcal{L}(V)$ ]; additionally, one needs to know all isomorphisms between the sections of $U$ and $V$.

Theorem 4.2.1 Given any isomorphism $\alpha: U_{1} / U_{2} \rightarrow V_{1} / V_{2}$ with $U_{2} \leq U_{1} \leq U$ and $V_{2} \leq V_{1} \leq V$, define

$$
[\alpha]=\left\{u+v \mid u \in U_{1}, v \in V_{1},\left(u+U_{2}\right) \alpha=v+V_{2}\right\}
$$

Then $[\alpha]$ is a submodule of $U \oplus V$, and

$$
\begin{array}{ll}
U \cap([\alpha]+V)=U_{1}, & U \cap[\alpha]=U_{2}, \\
(U+[\alpha]) \cap V=V_{1}, & {[\alpha] \cap V=V_{2} .}
\end{array}
$$

Conversely, given any submodule $W$ of $U \oplus V$, define $U_{1}, U_{2}, V_{1}, V_{2}$ by

$$
\begin{array}{ll}
U \cap(W+V)=U_{1}, & U \cap W=U_{2}, \\
(U+W) \cap V=V_{1}, & W \cap V=V_{2} .
\end{array}
$$

For each $u \in U_{1}$, the intersection $(u+W) \cap V$ is a coset of $V_{1}$ modulo $V_{2}$ which depends only on the coset $u+U_{2}$, and $\alpha: U_{1} / U_{2} \rightarrow V_{1} / V_{2}$ defined by

$$
\left(u+U_{2}\right) \alpha=(u+W) \cap V
$$

is an isomorphism such that $[\alpha]=W$. Consequently, $\alpha \mapsto[\alpha]$ is a bijection from the set of all isomorphisms of sections of $U$ to sections of $V$, to the set of submodules of $U \oplus V$.

Remark 4.2.2 The important special case of Theorem 4.2.1 which we will use is that the bijection $\alpha \mapsto[\alpha]$ also gives a one-to-one correspondence between isomorphisms of low sections of $U$ to low sections of $V$, to $\mathcal{V}(U \oplus V)$.

Remark 4.2.3 Let $U$ and $V$ be submodules of an $R$-module $W$ such that $U \cap V$ is finite. There is an obvious generalisation of Remark 4.2.2, giving a one-to-one correspondence between finite submodules of $W$ contained in $U+V$ and containing $U \cap V$, and isomorphisms between low sections $U_{1} / U_{2}$ of $U$ and low sections $V_{1} / V_{2}$ of $V$ such that $U \cap V \leq U_{2} \leq U_{1} \leq U$ and $U \cap V \leq V_{2} \leq V_{1} \leq V$. Where relevant in the sequel (see Propositions 4.4 .2 and 5.2.4), this generalisation is implicit in references to Theorem 4.2.1. 


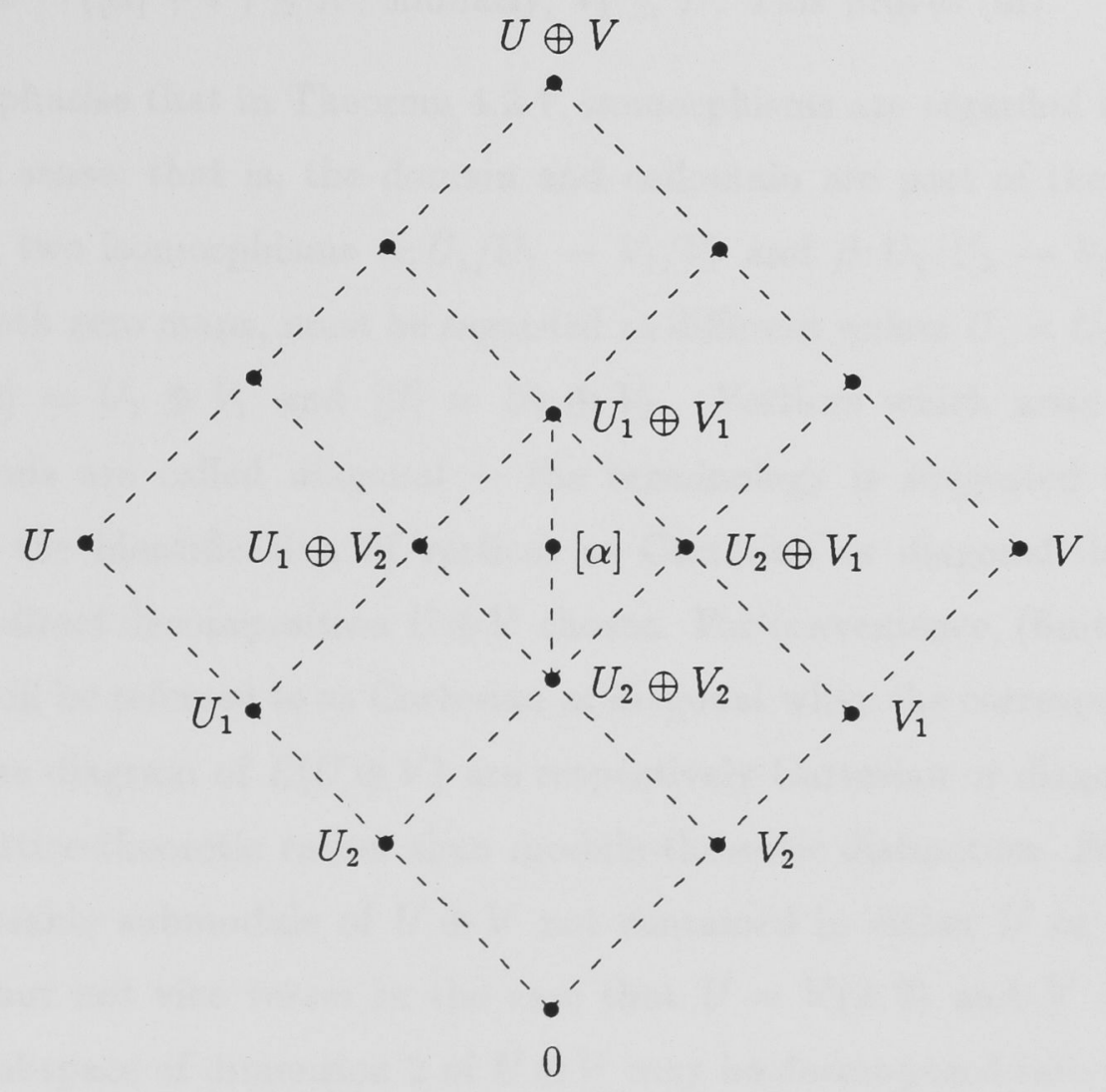

Figure 4.2: Illustration of Theorem 4.2.1

Using Theorem 4.2.1, we associate to each submodule $[\alpha]$ of $U \oplus V$ its unique "interval of definition" $\mathcal{L}\left(U_{1} \oplus V_{1} / U_{2} \oplus V_{2}\right)$. Remember that the Hasse diagram of $\mathcal{L}\left(U_{1} \oplus V_{1} / U_{2} \oplus V_{2}\right)$ is a subgraph of the Hasse diagram of $\mathcal{L}(U \oplus V)$. The following facts about intervals of definition follow readily from Theorem 4.2.1, and are stated here for ease of reference.

Proposition 4.2.4 Let $\alpha$ be an isomorphism of the section $U_{1} / U_{2}$ of $U$ onto the section $V_{1} / V_{2}$ of $V$.

(i) $[\alpha] \cap\left(U_{1} \oplus V_{2}\right)=[\alpha] \cap\left(U_{2} \oplus V_{1}\right)=U_{2} \oplus V_{2}$, $[\alpha]+\left(U_{1} \oplus V_{2}\right)=[\alpha]+\left(U_{2} \oplus V_{1}\right)=U_{1} \oplus V_{1}$.

(ii) $[\alpha] / U_{2} \oplus V_{2} \cong U_{1} / U_{2} \cong U_{1} \oplus V_{1} /[\alpha]$.

(iii) If $X \oplus Y \leq[\alpha]$ then $X \oplus Y \leq U_{2} \oplus V_{2}$ and if $[\alpha] \leq X \oplus Y$ then $U_{1} \oplus V_{1} \leq X \oplus Y$.

Proof. (i) follows from the modular law and (ii) follows from (i). If $X \oplus Y \leq[\alpha]$ then $X \leq[\alpha] \cap U=U_{2}$ and similarly $Y \leq V_{2}$. If $[\alpha] \leq X \oplus Y$ then $[\alpha]+V \leq X \oplus V$ 
and $U_{1}=U \cap([\alpha]+V) \leq X$; similarly, $V_{1} \leq Y$. This proves (iii).

We emphasise that in Theorem 4.2.1, isomorphisms are regarded in the category theoretical sense; that is, the domain and codomain are part of the definition. In particular, two isomorphisms $\alpha: U_{1} / U_{1} \rightarrow V_{1} / V_{1}$ and $\beta: U_{2} / U_{2} \rightarrow V_{2} / V_{2}$, although they are both zero maps, must be regarded as different unless $U_{1}=U_{2}$ and $V_{1}=V_{2}$ : indeed, $[\alpha]=U_{1} \oplus V_{1}$ and $[\beta]=U_{2} \oplus V_{2}$. Vertices which arise from nonzero isomorphisms are called diagonal - the terminology is suggested by Figure 4.2. Note that the identification of vertices as Cartesian or diagonal depends on the particular direct decomposition $U \oplus V$ chosen. For convenience, (finite) submodules of $U \oplus V$ will be referred to as Cartesian or diagonal when the corresponding vertices in the Hasse diagram of $\mathcal{L}(U \oplus V)$ are respectively Cartesian or diagonal. However, this is a lattice-theoretic rather than module-theoretic distinction. For example, an indecomposable submodule of $U \oplus V$ not contained in either $U$ or $V$ is certainly diagonal, but not vice versa: in the case that $U=V(2,2)$ and $V=V(1,2)$, any diagonal subspace of dimension 2 of $U \oplus V$ may be decomposed into the direct sum of two 1-dimensional subspaces.

A modular lattice is distributive if and only if it does not contain a sublattice with the same Hasse diagram as $\mathcal{L}(U)$ in Figure 4.1 (see Grätzer (1971), p.70, Theorem 2). Therefore, for general $U$ and $V, \mathcal{L}(U \oplus V)$ is distributive if and only if $\mathcal{L}(U)$ and $\mathcal{L}(V)$ are distributive and the Hasse diagram of $\mathcal{L}(U \oplus V)$ has no diagonal vertices. In that case, $\mathcal{L}(U \oplus V)=\mathcal{L}(U) \times \mathcal{L}(V)$. The Hasse diagrams of the submodule lattices that we consider in Chapter 5 (and in the analogous situation for $T=C$ ) have many diagonal vertices.

\subsection{The Hasse diagram of $\mathcal{L}(U \oplus V)$}

In this section, we carry out a classification of the elements of $\mathcal{E}(U \oplus V)$. Specifically, we define three distinct types of edges and show that each element of $\mathcal{E}(U \oplus V)$ is one of these types. This is analogous to the classification of elements of $\mathcal{V}(U \oplus V)$ into two distinct types, Cartesian and diagonal, carried out in Section 4.2. Here, also, the classification depends on the direct decomposition $U \oplus V$ chosen.

The three distinct types of edges by which we classify the elements of $\mathcal{E}(U \oplus V)$ are called Cartesian, restriction and composition edges. Cartesian edges were defined 
in Section 4.2. The existence of restriction edges is implicit in Theorem 4.2.1; the construction of edges of this type is described explicitly in Proposition 4.3.1. The construction of composition edges is described in Proposition 4.3.3. A restriction edge has at least one diagonal endpoint. On the other hand, both endpoints of a composition edge are diagonal. Remark 4.3.6 provides a criterion to determine whether an edge with diagonal endpoints is restriction or composition.

Proposition 4.3.1 Let $\alpha$ be an isomorphism of the section $U_{1} / U_{2}$ of $U$ onto the section $V_{1} / V_{2}$ of $V$. Suppose that $U_{2} \leq U^{\prime} \leq U_{1}$ and set $V^{\prime} / V_{2}=\left(U^{\prime} / U_{2}\right) \alpha$. Define isomorphisms $\underline{\alpha}: U^{\prime} / U_{2} \rightarrow V^{\prime} / V_{2}$ and $\bar{\alpha}: U_{1} / U^{\prime} \rightarrow V_{1} / V^{\prime}$ by

$$
\underline{\alpha}=\left.\alpha\right|_{U^{\prime} / U_{2}}
$$

and

$$
\left(u+U^{\prime}\right) \bar{\alpha}=v+V^{\prime}
$$

for $u \in U_{1}$, where $\left(u+U_{2}\right) \alpha=v+V_{2}$. Then

(i) $[\alpha] \cap\left(U^{\prime} \oplus V^{\prime}\right)=[\underline{\alpha}]$ and $[\alpha]+\left(U^{\prime} \oplus V^{\prime}\right)=[\bar{\alpha}]$,

(ii) $[\alpha] /[\underline{\alpha}] \cong U_{1} / U^{\prime}$ and $[\bar{\alpha}] /[\alpha] \cong U^{\prime} / U_{2}$.

Proof. Clearly, $[\underline{\alpha}] \leq[\alpha]$ and $[\alpha] \leq[\bar{\alpha}]$, by the definitions of $[\alpha],[\underline{\alpha}]$ and $[\bar{\alpha}]$ given by Theorem 4.2.1. The situation is illustrated in Figure 4.3.

(i) By the modular law and (i) of Proposition 4.2.4,

$$
\begin{aligned}
{[\alpha] \cap\left(U^{\prime} \oplus V^{\prime}\right) } & =[\alpha] \cap\left([\underline{\alpha}]+\left(U^{\prime} \oplus V_{2}\right)\right) \\
& =[\underline{\alpha}]+\left([\alpha] \cap\left(U^{\prime} \oplus V_{2}\right)\right) \\
& =[\underline{\alpha}]+\left(U_{2} \oplus V_{2}\right) \\
& =[\underline{\alpha}] .
\end{aligned}
$$

The other equality is proved similarly.

(ii) By (i) and Proposition 4.2 .4 (ii),

$$
\begin{aligned}
{[\alpha] /[\underline{\alpha}] } & =[\alpha] /[\alpha] \cap\left(U^{\prime} \oplus V^{\prime}\right) \\
& \cong[\alpha]+\left(U^{\prime} \oplus V^{\prime}\right) / U^{\prime} \oplus V^{\prime} \\
& =[\bar{\alpha}] / U^{\prime} \oplus V^{\prime} \\
& \cong U_{1} / U^{\prime} .
\end{aligned}
$$

The other isomorphism is proved similarly. 


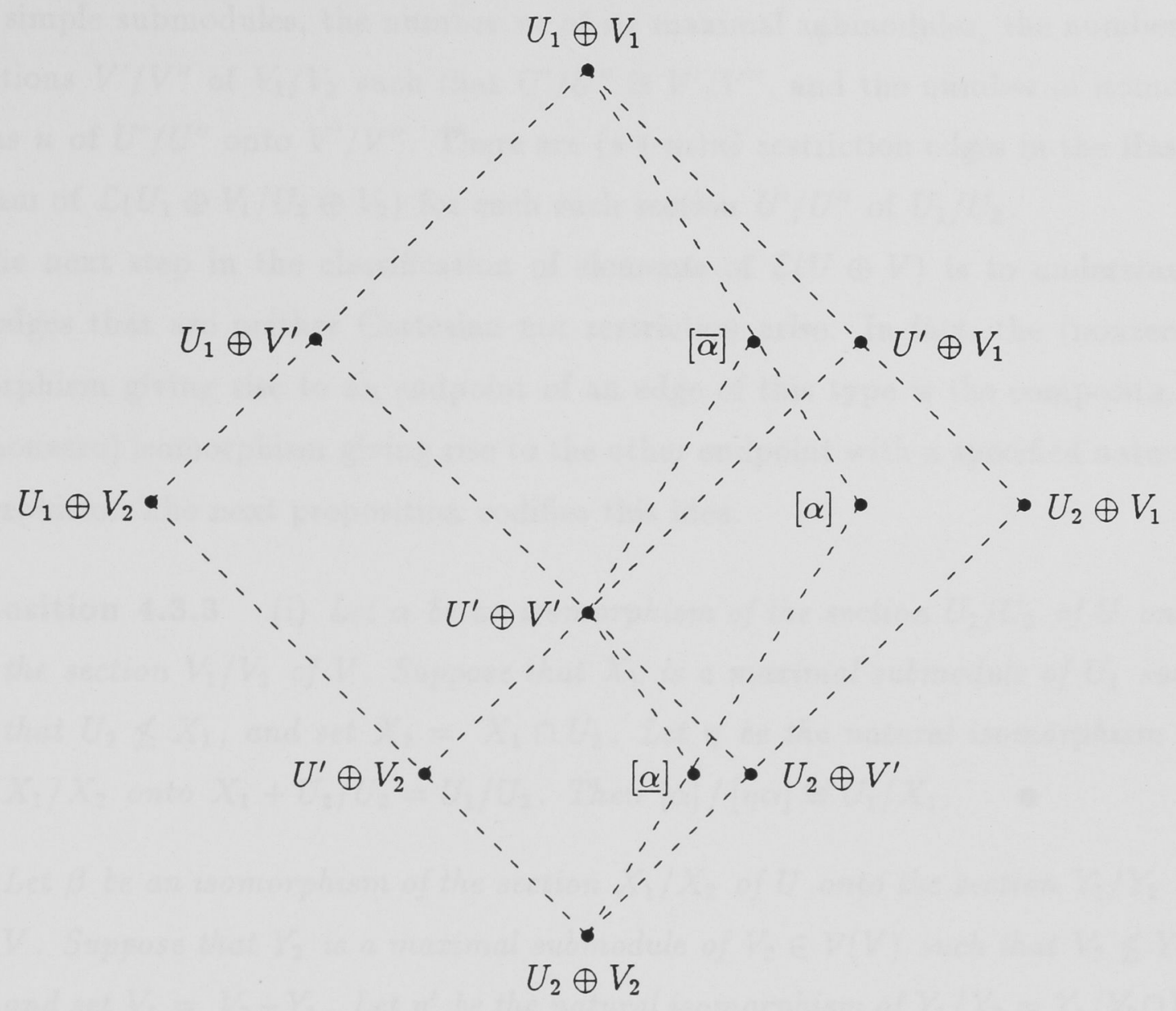

Figure 4.3: Illustration of Proposition 4.3.1

Remark 4.3.2 The notation $\underline{\alpha}$ and $\bar{\alpha}$ suppresses dependence on the choice of $U^{\prime}$. However, this choice will usually be clear from the context.

In Proposition 4.3.1, suppose that $U_{1} / U_{2}$ is low. Then $[\alpha] /[\underline{\alpha}] \in \mathcal{E}(U \oplus V)$ if and only if $U_{1} / U^{\prime} \in \mathcal{E}(U)$ and $[\bar{\alpha}] /[\alpha] \in \mathcal{E}(U \oplus V)$ if and only if $U^{\prime} / U_{2} \in \mathcal{E}(U)$. In either of these situations, $[\alpha] /[\underline{\alpha}]$ and $[\bar{\alpha}] /[\alpha]$ are called restriction edges. The justification for the terminology is that $\bar{\alpha}$ is the "restriction" of $[\alpha]$ to the section $U_{1} / U^{\prime}$ of $U_{1} / U_{2}$ and $\underline{\alpha}$ is the restriction to the section $U^{\prime} / U_{2}$. Note that $[\bar{\alpha}]$ or $[\underline{\alpha}]$ may be Cartesian vertices. A restriction edge is not Cartesian. For if $[\alpha] /[\underline{\alpha}]$ or $[\bar{\alpha}] /[\alpha]$ were Cartesian then we would have $U_{1}=U_{2}$. But by convention, the zero module $U_{1} / U_{1}$ has no maximal or simple submodules.

We may construct restriction edges in the Hasse diagram of the interval of definition $\mathcal{L}\left(U_{1} \oplus V_{1} / U_{2} \oplus V_{2}\right)$ of $[\alpha]$ inductively, using the following count. For each integer $l \geq 1$, list all sections $U^{\prime} / U^{\prime \prime}$ of $U_{1} / U_{2}$ of composition length $l$ which are isomorphic to a section of $V_{1} / V_{2}$. For each such section $U^{\prime} / U^{\prime \prime}$, count the number $s$ 
of its simple submodules, the number $m$ of its maximal submodules, the number $i$ of sections $V^{\prime} / V^{\prime \prime}$ of $V_{1} / V_{2}$ such that $U^{\prime} / U^{\prime \prime} \cong V^{\prime} / V^{\prime \prime}$, and the number of isomorphisms $n$ of $U^{\prime} / U^{\prime \prime}$ onto $V^{\prime} / V^{\prime \prime}$. There are $(s+m) n i$ restriction edges in the Hasse diagram of $\mathcal{L}\left(U_{1} \oplus V_{1} / U_{2} \oplus V_{2}\right)$ for each such section $U^{\prime} / U^{\prime \prime}$ of $U_{1} / U_{2}$.

The next step in the classification of elements of $\mathcal{E}(U \oplus V)$ is to understand how edges that are neither Cartesian nor restriction arise. In fact, the (nonzero) isomorphism giving rise to an endpoint of an edge of this type is the composite of the (nonzero) isomorphism giving rise to the other endpoint with a specified natural isomorphism. The next proposition codifies this idea.

Proposition 4.3.3 (i) Let $\alpha$ be an isomorphism of the section $U_{1} / U_{2}$ of $U$ onto the section $V_{1} / V_{2}$ of $V$. Suppose that $X_{1}$ is a maximal submodule of $U_{1}$ such that $U_{2} \not X_{1}$, and set $X_{2}=X_{1} \cap U_{2}$. Let $\eta$ be the natural isomorphism of $X_{1} / X_{2}$ onto $X_{1}+U_{2} / U_{2}=U_{1} / U_{2}$. Then $[\alpha] /[\eta \alpha] \cong U_{1} / X_{1}$.

(ii) Let $\beta$ be an isomorphism of the section $X_{1} / X_{2}$ of $U$ onto the section $Y_{1} / Y_{2}$ of $V$. Suppose that $Y_{2}$ is a maximal submodule of $V_{2} \in \mathcal{V}(V)$ such that $V_{2} \not Y_{1}$, and set $V_{1}=V_{2}+Y_{1}$. Let $\eta^{\prime}$ be the natural isomorphism of $Y_{1} / Y_{2}=Y_{1} / V_{2} \cap Y_{1}$ onto $V_{1} / V_{2}$. Then $\left[\beta \eta^{\prime}\right] /[\beta] \cong V_{2} / Y_{2}$.

Proof. The proof proceeds similarly in both cases and so we prove only (i). See Figure 4.4 .

It is clear from the definitions of $[\alpha]$ and $[\eta \alpha]$ given by Theorem 4.2 .1 that $[\alpha] \cap\left(X_{1} \oplus V_{1}\right)=[\eta \alpha]$. Since $X_{1} \oplus V_{1} \leq[\alpha]+\left(X_{1} \oplus V_{1}\right) \leq U_{1} \oplus V_{1}$ and $U_{1} \oplus V_{1} / X_{1} \oplus V_{1}$ is simple, either $[\alpha] \leq X_{1} \oplus V_{1}$ or $[\alpha]+\left(X_{1} \oplus V_{1}\right)=U_{1} \oplus V_{1}$. By Proposition 4.2.4 (iii), the first possibility implies that $U_{1}=X_{1}$ and so is excluded. The second implies that $[\alpha] /[\eta \alpha] \cong[\alpha] /[\alpha] \cap\left(X_{1} \oplus V_{1}\right) \cong U_{1} \oplus V_{1} / X_{1} \oplus V_{1} \cong U_{1} / X_{1}$.

Remark 4.3.4 In Proposition 4.3.3 (i), $U_{1} / X_{1}$ is said to be perspective to $U_{2} / X_{2}$; in (ii), $V_{1} / Y_{1}$ is perspective to $V_{2} / Y_{2}$.

If $U_{1} / U_{2}$ is low and $\alpha \neq 0$ in Proposition 4.3 .3 (i) then $[\alpha] /[\eta \alpha]$ is called a composition edge; if $X_{1} / X_{2}$ is low and $\beta \neq 0$ in (ii) then $\left[\beta \eta^{\prime}\right] /[\beta]$ is also called a composition edge. Note that these edges have diagonal endpoints and hence are not Cartesian. Nor is a composition edge a restriction edge: in (i), we stipulate that $X_{1} / X_{2}$ is not a section of $U_{1} / U_{2}$, and in (ii) that $Y_{1} / Y_{2}$ is not a section of $V_{1} / V_{2}$. 
Also, a composition edge arising as in (i) cannot arise as in (ii) and vice versa. In Figure 4.4, the sections drawn as unbroken lines in each picture are pairwise isomorphic, as are all sections drawn as broken lines.
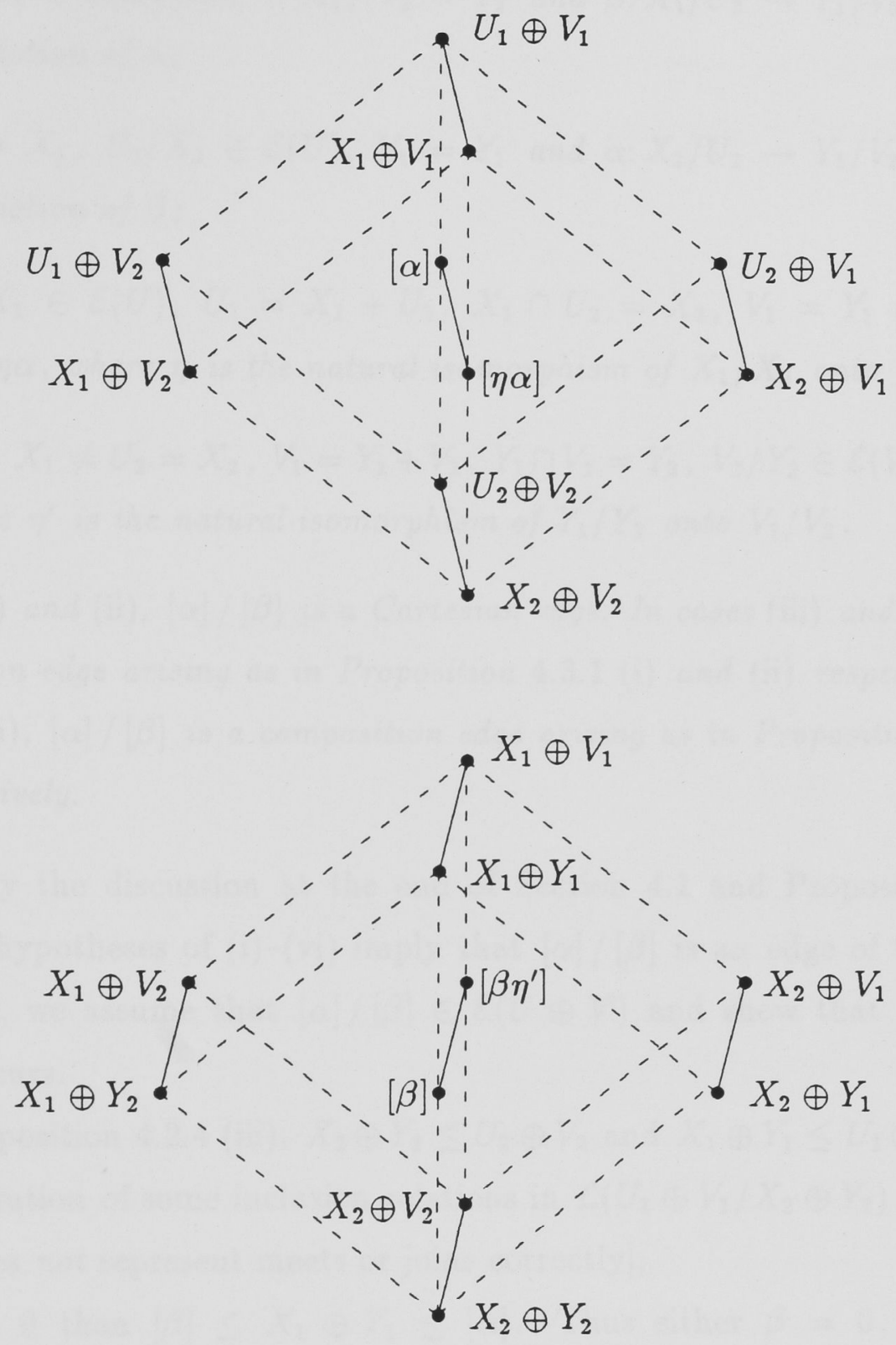

Figure 4.4: Illustration of Proposition 4.3.3

In the next result we show that each element of $\mathcal{E}(U \oplus V)$ is one of the three distinct types defined above.

Theorem 4.3.5 Given an isomorphism $\alpha$ of the low section $U_{1} / U_{2}$ of $U$ onto the section $V_{1} / V_{2}$ of $V$, and an isomorphism $\beta$ of the low section $X_{1} / X_{2}$ of $U$ onto the section $Y_{1} / Y_{2}$ of $V,[\alpha] /[\beta] \in \mathcal{E}(U \oplus V)$ if and only if $U_{1} \geq X_{1}, U_{2} \geq X_{2}$, $V_{1} \geq Y_{1}, V_{2} \geq Y_{2}$ and one of the following holds: 
(i) $U_{1} / X_{1} \in \mathcal{E}(U)$ and $V_{1}=Y_{1}=V_{2}=Y_{2}$;

(ii) $U_{1}=X_{1}=U_{2}=X_{2}$ and $V_{1} / Y_{1} \in \mathcal{E}(V)$;

(iii) $U_{1} / X_{1} \in \mathcal{E}(U), U_{2}=X_{2}, V_{2}=Y_{2}$ and $\beta: X_{1} / U_{2} \rightarrow Y_{1} / V_{2}$ is the relevant restriction of $\alpha$;

(iv) $U_{1}=X_{1}, U_{2} / X_{2} \in \mathcal{E}(U), V_{1}=Y_{1}$ and $\alpha: X_{1} / U_{2} \rightarrow Y_{1} / V_{2}$ is the relevant restriction of $\beta$;

(v) $U_{1} / X_{1} \in \mathcal{E}(U), U_{1}=X_{1}+U_{2}, X_{1} \cap U_{2}=X_{2}, V_{1}=Y_{1} \neq V_{2}=Y_{2}$ and $\beta=\eta \alpha$, where $\eta$ is the natural isomorphism of $X_{1} / X_{2}$ onto $U_{1} / U_{2}$;

(vi) $U_{1}=X_{1} \neq U_{2}=X_{2}, V_{1}=Y_{1}+V_{2}, Y_{1} \cap V_{2}=Y_{2}, V_{2} / Y_{2} \in \mathcal{E}(V)$ and $\alpha=\beta \eta^{\prime}$, where $\eta^{\prime}$ is the natural isomorphism of $Y_{1} / Y_{2}$ onto $V_{1} / V_{2}$.

In cases (i) and (ii), $[\alpha] /[\beta]$ is a Cartesian edge. In cases (iii) and (iv), $[\alpha] /[\beta]$ is a restriction edge arising as in Proposition 4.3 .1 (i) and (ii) respectively. In cases (v) and (vi), $[\alpha] /[\beta]$ is a composition edge arising as in Proposition 4.3 .3 (i) and (ii) respectively.

Proof. By the discussion at the end of Section 4.1 and Propositions 4.3 .1 and 4.3.3, the hypotheses of (i)-(vi) imply that $[\alpha] /[\beta]$ is an edge of the stated type. Conversely, we assume that $[\alpha] /[\beta] \in \mathcal{E}(U \oplus V)$ and show that precisely one of (i) - (vi) occurs.

By Proposition 4.2.4 (iii), $X_{2} \oplus Y_{2} \leq U_{2} \oplus V_{2}$ and $X_{1} \oplus Y_{1} \leq U_{1} \oplus V_{1}$. Figure 4.5 is an illustration of some inclusion relations in $\mathcal{L}\left(U_{1} \oplus V_{1} / X_{2} \oplus Y_{2}\right)$ [in general, this picture does not represent meets or joins correctly].

If $\alpha=0$ then $[\beta] \leq X_{1} \oplus Y_{1} \leq[\alpha]$. Thus either $\beta=0$, or $\beta \neq 0$ and $[\alpha]=X_{1} \oplus Y_{1} \in \mathcal{L}\left(X_{1} \oplus Y_{1} / X_{2} \oplus Y_{2}\right)$. Similarly, if $\beta=0$ then either $\alpha=0$, or $\alpha \neq 0$ and $[\beta]=U_{2} \oplus V_{2} \in \mathcal{L}\left(U_{1} \oplus V_{1} / U_{2} \oplus V_{2}\right)$. When $\alpha=\beta=0,[\alpha] /[\beta]$ is a Cartesian edge and one of (i) or (ii) occurs. When $\alpha \neq 0$ and $[\beta] \in \mathcal{L}\left(U_{1} \oplus V_{1} / U_{2} \oplus V_{2}\right)$, we have $U_{2} \oplus V_{2}=X_{2} \oplus Y_{2}$ by Proposition 4.2 .4 (iii). Let $\underline{\alpha}$ be the restriction of $\alpha$ to the section $X_{1} / U_{2}$ of $U_{1} / U_{2}$. Since $[\beta] \leq[\alpha]$, it follows from Theorem 4.2.1 that $[\beta] \leq[\underline{\alpha}]$. That is, $[\beta] \leq[\underline{\alpha}] \leq[\alpha]$ and thus $[\beta]=[\underline{\alpha}]$. For otherwise we would have $U_{1}=X_{1}$ and then

$$
[\alpha] /[\beta] \cong \frac{[\alpha] / U_{2} \oplus V_{2}}{[\beta] / X_{2} \oplus Y_{2}}
$$




$$
\begin{aligned}
& \cong \frac{U_{1} / U_{2}}{X_{1} / X_{2}} \\
& =0,
\end{aligned}
$$

where the second isomorphism follows from Proposition 4.2.4 (ii). Therefore, $\beta=\underline{\alpha}$ and by Proposition 4.3.1 (ii), $[\alpha] /[\beta] \cong U_{1} / X_{1}$. These are the conditions of (iii). When $[\alpha] \in \mathcal{L}\left(X_{1} \oplus Y_{1} / X_{2} \oplus Y_{2}\right)$ and $\beta \neq 0$, by similar reasoning we derive the conditions of (iv).

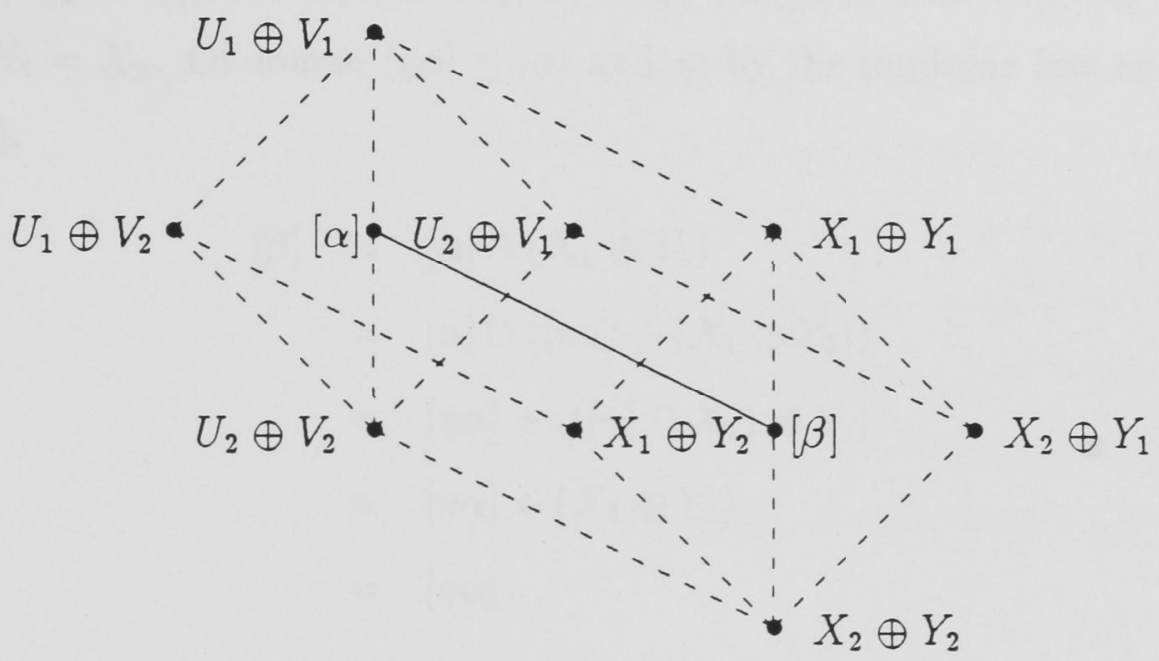

Figure 4.5: Some inclusion relations in the interval $\mathcal{L}\left(U_{1} \oplus V_{1} / X_{2} \oplus Y_{2}\right)$

From now on, assume that $\alpha$ and $\beta$ are nonzero, $[\alpha] \notin \mathcal{L}\left(X_{1} \oplus Y_{1} / X_{2} \oplus Y_{2}\right)$ and $[\beta] \notin \mathcal{L}\left(U_{1} \oplus V_{1} / U_{2} \oplus V_{2}\right)$. This means that $[\alpha] /[\beta]$ is neither a Cartesian nor a restriction edge. With these assumptions, we will show that $[\alpha] /[\beta]$ is a composition edge (and then Figure 4.5 does represent joins and meets correctly).

It is a consequence of the assumptions on $\alpha$ and $\beta$ that $[\alpha] \cap\left(X_{1} \oplus Y_{1}\right)=[\beta]$ and $[\beta]+\left(U_{2} \oplus V_{2}\right)=[\alpha]$. Therefore, $U_{2} \oplus V_{2} /[\beta] \cap\left(U_{2} \oplus V_{2}\right)$ is simple. Also, $[\beta] \cap\left(U_{2} \oplus V_{2}\right) \leq\left(X_{1} \cap U_{2}\right) \oplus\left(Y_{1} \cap V_{2}\right) \leq U_{2} \oplus V_{2}$. If $U_{2} \leq X_{1}$ and $V_{2} \leq Y_{1}$ then

$$
\begin{aligned}
{[\beta] \cap\left(U_{2} \oplus V_{2}\right) } & =[\alpha] \cap\left(X_{1} \oplus Y_{1}\right) \cap\left(U_{2} \oplus V_{2}\right) \\
& =[\alpha] \cap\left(U_{2} \oplus V_{2}\right) \\
& =U_{2} \oplus V_{2},
\end{aligned}
$$

which contradicts the assumption that $[\beta] \notin \mathcal{L}\left(U_{1} \oplus V_{1} / U_{2} \oplus V_{2}\right)$. Thus $[\beta] \cap\left(U_{2} \oplus V_{2}\right)$ is Cartesian and so, by Proposition 4.2.4 (iii), $[\beta] \cap\left(U_{2} \oplus V_{2}\right)=X_{2} \oplus Y_{2}$. Furthermore $X_{1} \cap U_{2}=X_{2}$ and $Y_{1} \cap V_{2}=Y_{2}$. Similarly $[\alpha]+\left(X_{1} \oplus Y_{1}\right)=U_{1} \oplus V_{1}, X_{1}+U_{2}=U_{1}$ 
and $Y_{1}+V_{2}=V_{1}$. Then it is clear that

$$
[\alpha] /[\beta] \cong U_{1} \oplus V_{1} / X_{1} \oplus Y_{1} \cong U_{2} \oplus V_{2} / X_{2} \oplus Y_{2}
$$

By (4.1), either $V_{1}=Y_{1}$ and $V_{2}=Y_{1} \cap V_{2}=Y_{2}$, or $U_{1}=X_{1}$ and $U_{2}=X_{1} \cap U_{2}=X_{2}$. If $V_{2}=Y_{2}$ then, by $(4.1), U_{1} / X_{1} \cong X_{1}+U_{2} / X_{1} \cong U_{2} / X_{2} \cong[\alpha] /[\beta]$ and so $V_{1}=Y_{1}$. Thus $V_{1}=Y_{1}$ if and only if $V_{2}=Y_{2}$ and, similarly, $U_{1}=X_{1}$ if and only if $U_{2}=X_{2}$.

If $V_{1}=Y_{1}$ and $V_{2}=Y_{2}$ then $U_{1} / X_{1}, U_{2} / X_{2} \in \mathcal{E}(U)$. Let $\eta$ be the natural isomorphism of $X_{1} / X_{2}=X_{1} / X_{1} \cap U_{2}$ onto $U_{1} / U_{2}=X_{1}+U_{2} / U_{2}$. Now $[\alpha] \cap X_{1} \leq U_{2} \cap X_{1}$; thus, $[\alpha] \cap X_{1}=X_{2}$. Of course $[\eta \alpha] \leq[\alpha]$ and so by the modular law and Proposition $4.2 .4(\mathrm{i})$,

$$
\begin{aligned}
{[\beta] } & =[\alpha] \cap\left(X_{1} \oplus V_{1}\right) \\
& =[\alpha] \cap\left([\eta \alpha]+\left(X_{1} \oplus Y_{2}\right)\right) \\
& =[\eta \alpha]+\left(\left([\alpha] \cap X_{1}\right) \oplus Y_{2}\right) \\
& =[\eta \alpha]+\left(X_{2} \oplus Y_{2}\right) \\
& =[\eta \alpha] .
\end{aligned}
$$

We have derived the conditions of (v). Similarly, if $U_{1}=X_{1}$ and $U_{2}=X_{2}$, we derive the conditions of (vi).

In practice, to obtain a description of the Hasse diagram of $\mathcal{L}(U \oplus V)$, one should first list the elements of $\mathcal{V}(U \oplus V)$ via Remak's theorem. The module-theoretic information required for this determination, together with knowledge of $\mathcal{E}(U)$ and $\mathcal{E}(V)$, is then essentially all that is needed to list the elements of $\mathcal{E}(U \oplus V)$ via Theorem 4.3.5.

The next result was referred to before Proposition 4.3.1.

Proposition 4.3.6 Let $\alpha$ and $\beta$ be as in the hypotheses of Theorem 4.3.5 and also suppose that $[\alpha]$ and $[\beta]$ are non-Cartesian submodules and $[\alpha] /[\beta] \in \mathcal{E}(U \oplus V)$. Then $[\alpha] /[\beta]$ is a composition edge if and only if $\left|U_{1} / U_{2}\right|=\left|X_{1} / X_{2}\right|$.

Proof. This follows from facts observed in the proof of Theorem 4.3.5: if $[\alpha] /[\beta]$ is a restriction edge, then either $U_{1} / X_{1} \in \mathcal{E}(U)$ and $U_{2}=X_{2}$, or $U_{2} / X_{2} \in \mathcal{E}(U)$ and $U_{1}=X_{1}$; if $[\alpha] /[\beta]$ is a composition edge then $U_{1} / X_{1} \cong U_{2} / X_{2}$.

The classification of edges leads naturally to a description of the Hasse diagram of $\mathcal{L}(U \oplus V)$. Each element of $\mathcal{V}(U \oplus V)$ is contained in an interval with Cartesian 
endpoints; namely, the interval of definition of the vertex. By Proposition 4.2.4 (iii), the interval of definition is in fact the unique minimal interval of $\mathcal{L}(U \oplus V)$ with Cartesian endpoints containing the vertex. Hence, for each element of $\mathcal{E}(U \oplus V)$, there is a unique minimal interval of $\mathcal{L}(U \oplus V)$ with Cartesian endpoints containing the endpoints of the edge. Together with $\mathcal{L}(U) \times \mathcal{L}(V)$, we call these minimal intervals the pages in an atlas of $\mathcal{L}(U \oplus V)$ [this terminology was suggested to me by Dr L. G. Kovács]. Note that the non-Cartesian product pages are finite, whereas $\mathcal{L}(U) \times \mathcal{L}(V)$ is not necessarily finite and in general is not an interval. Since a finite (nonzero) $R$-module has a maximal submodule, each element of $\mathcal{V}(U \oplus V)$ is the endpoint of at least one element of $\mathcal{E}(U \oplus V)$. Also, the Hasse diagram of each page is a subgraph and so the union of the Hasse diagrams of the pages (that is, the set unions of their vertex sets and edge sets) is the whole Hasse diagram of $\mathcal{L}(U \oplus V)$. The Cartesian product subgraph is well understood in terms of the Hasse diagrams of $\mathcal{L}(U)$ and $\mathcal{L}(V)$, so that the problem of describing the Hasse diagram of $\mathcal{L}(U \oplus V)$ is reduced essentially to drawing the Hasse diagrams of the (finite) non-Cartesian product pages.

The pages are described implicitly in Theorem 4.3.5; these descriptions in turn suggest how we may list the pages in practice. By designating $\mathcal{L}(U) \times \mathcal{L}(V)$ as a page, we only need to list the pages chosen for edges that are either restriction or composition. Clearly, the minimal interval with Cartesian endpoints containing the endpoints of a restriction edge is the interval of definition of one of the edge's endpoints. Thus, for each 4-tuple $\left(U_{1}, U_{2}, V_{1}, V_{2}\right)$, where $U_{1} / U_{2}$ is a nonzero low section of $U$ that is isomorphic to the section $V_{1} / V_{2}$ of $V$, we list $\mathcal{L}\left(U_{1} \oplus V_{1} / U_{2} \oplus V_{2}\right)$ as a page. The relevant page for a composition edge is one of two possible types. Consider the 5-tuple $\left(U_{1}, U_{2}, V_{1}, V_{2}, M\right)$, where $U_{1} / U_{2}$ is a nonzero low section of $U$ isomorphic to the section $V_{1} / V_{2}$ of $V$, and one of the following situations occurs:

- $M$ is a submodule of $U$ such that $U_{1} / M \in \mathcal{E}(U)$;

- $M$ is a submodule of $V$ such that $M / V_{2} \in \mathcal{E}(V)$.

For each such 5 -tuple we list as a page $\mathcal{L}\left(U_{1} \oplus V_{1} /\left(U_{2} \cap M\right) \oplus V_{2}\right)$ in the first situation if and only if $U_{2} \not \leq M$, and $\mathcal{L}\left(\left(U_{1} \oplus V_{1}\right)+M / U_{2} \oplus V_{2}\right)$ in the second situation if and only if $M \not V_{1}$. [Here, we are counting pairs of perspective edges in the Hasse diagrams of $\mathcal{L}(U)$ and $\mathcal{L}(V)$.] The first step in listing such 5 -tuples is to consider each 4-tuple $\left(U_{1}, U_{2}, V_{1}, V_{2}\right)$ and determine the maximal submodules of $U_{1}\left(V_{1}\right)$ and 
the submodules of $U(V)$ in which $U_{2}\left(V_{2}\right)$ is maximal. Of course, the maximal submodules of $U_{1}$ containing $U_{2}$ (respectively, the submodules of $U_{1}$ in which $U_{2}$ is maximal) are bijectively paired to the maximal submodules of $V_{1}$ containing $V_{2}$ (respectively, the submodules of $V_{1}$ in which $V_{2}$ is maximal). The point is that in compiling the list of 5 -tuples in this way, the only extra information needed to list the edges in each page is the number of isomorphisms between $U_{1} / U_{2}$ and $V_{1} / V_{2}$, for each 4-tuple $\left(U_{1}, U_{2}, V_{1}, V_{2}\right)$.

The above procedure yields all pages in an atlas (and by extension, all edges in a page). However, the procedure may not be feasible unless certain conditions are satisfied. For instance, it is necessary that the Hasse diagrams of $\mathcal{L}(U)$ and $\mathcal{L}(V)$ are uncomplicated and well understood (otherwise there may be difficulty even in constructing the Cartesian product page). Also, it is clear that the reduction to pages is not useful if the domains of isomorphisms between low sections of $U$ and $V$ have "large" composition length, since then the pages and their Hasse diagrams are also large. For example, if $U$ is finite and $U \cong V$ then it is possible that $\mathcal{L}(U \oplus V)$ is page in its own atlas. Another complication arises if there are many (possibly infinitely many) pages. In general, therefore, we stipulate that the domains of isomorphisms between low sections of $U$ and $V$ fall into a "small" number of isomorphism classes and that these domains have small automorphism groups. Then, even if there are many pages, their Hasse diagrams fall into a small number of isomorphism classes.

The listing of pages will almost always yield some redundancies, in the sense that some pages are sublattices of other pages. It is desirable to recognise and then delete redundant pages. This is possible if the conditions on domains of isomorphisms between low sections of $U$ and $V$ mentioned above are satisfied, so that the nonCartesian product pages are small intervals and we may compare these pages for containment by an essentially (reverse) inductive procedure. (To determine whether one non-Cartesian product page is contained in another, we refer to the Cartesian product subgraph.) When all redundant pages have been deleted, the remaining atlas is complete and irredundant: the minimal condition for pages ensures that each element of $\mathcal{E}(U \oplus V)$ occurs in the Hasse diagram of at least one page, and there is at least one edge in the Hasse diagram of each page (with the possible exception of the Cartesian product page) which does not occur in the Hasse diagram of any other page. In practical terms, this atlas may be constructed by choosing one 
representative from each of the isomorphism classes of the Hasse diagrams of the irredundant pages. In these diagrams one needs to be able to identify the vertices as submodules of $U \oplus V$, so that a diagram should have labelled vertices. The labelling scheme chosen may influence the choice of pages in the atlas.

\subsection{Examples}

The motivation for this chapter arose from the need to describe the poset of finite normal subgroups in the base group of $C_{2 \infty}$ wr $V_{4}$. Indeed, the summands in the relevant direct decomposition for this example satisfy all the conditions (small domains of isomorphisms, etc.) outlined at the end of Section 4.3, required for the reduction to pages to be a useful one. In this section, we consider several lattices which in fact occur as pages in a complete and irredundant atlas in this example.

1. Let $A$ be the group algebra of the cyclic group $\left\langle x \mid x^{p}=1\right\rangle$ over the $\operatorname{ring} \mathbb{Z} / p^{n} \mathbb{Z}$, where $p$ is a prime and $n \geq 1$. We will describe the Hasse diagram of $\mathcal{L}\left(A_{A}\right)$. By Proposition 2.1.2, this means describing the Hasse diagram of $\mathcal{L}(\operatorname{rad} A / \operatorname{soc} A)$. Also by Proposition 2.1.2, there is a unique isomorphism type of simple $A$-module. Let $Z$ be the submodule spanned by the trace element $t=\sum_{i=0}^{p-1} x^{i}$ and $W$ be the augmentation ideal of $A$. By Proposition 2.1.6, $\operatorname{rad} A=Z+W$. Also, $Z \cap W=p^{n-1} Z=\operatorname{soc} A$. Therefore,

$$
\operatorname{rad} A / \operatorname{soc} A=Z / \operatorname{soc} A \oplus W / \operatorname{soc} A
$$

To describe the Hasse diagram of $\mathcal{L}(\operatorname{rad} A / \operatorname{soc} A)$, we determine the Hasse diagrams of $\mathcal{L}(Z / \operatorname{soc} A)$ and $\mathcal{L}(W / \operatorname{soc} A)$ and apply the theory of this chapter to (4.2).

Of course, $Z$ is just a cyclic group of order $p^{n}$ upon which $x$ acts trivially. Thus, $Z / \operatorname{soc} A$ is a uniserial $A$-module of composition length $n-1$. The $A$-module structure of $W$ is given next.

Lemma 4.4.1 $W$ is a uniserial A-module of composition length $(p-1) n$, with composition terms $W_{i}$ defined inductively by $W_{0}=\{0\}$ and $W_{i}=\left\langle w_{i}, W_{i-1}\right\rangle$ for $1 \leq i \leq(p-1) n$, where $w_{i}=(1-x)^{(p-1) n-i+1}$. Also, the only sections of $W$ upon which $x$ acts trivially are the composition factors $W_{i} / W_{i-1}$.

Proof. First note that

$$
p\left(1-x^{k}\right) \in W^{2}
$$


and

$$
1-x^{k} \equiv k(1-x) \bmod W^{2}
$$

for $1 \leq k \leq p-1$ : (4.3) follows from $1=\left(1-\left(1-x^{k}\right)\right)^{p} \equiv 1-p\left(1-x^{k}\right) \bmod W^{2}$, and (4.4) is proved by induction on $k$, using the identity

$$
(1-a b)=(1-a)+(1-b)-(1-a)(1-b)
$$

By Proposition 2.1.1 and (4.3), $\operatorname{rad} W=p W+W^{2}=W^{2}$, and, by induction on $i, \operatorname{rad}^{i} W=W^{i+1}$. By (4.4), W/W is a cyclic group of order $p$, generated by $(1-x)+W^{2}$. Again by induction on $i, W^{i} / W^{i+1}$ is cyclic of order $p$, generated by $(1-x)^{i}+W^{i+1}$. Therefore, the Loewy factors $\operatorname{rad}^{i} W / \operatorname{rad}^{i+1} W$ of $W$ are simple and $W$ is uniserial. Since $(1-x)(1-x)^{i} \in W^{i+1} \backslash W^{j}$ for $j>i+1$, we see that $x$ acts trivially on the Loewy factors and non-trivially on all other sections of $W$.

In the statement of Lemma 4.4.1, the composition terms of $W$ are numbered in reverse order. This is to avoid difficulties with notation in the next proposition.

Proposition 4.4.2 Define $z_{i}=p^{n-i} t$ and recall the definition of $w_{i}$ and $W_{i}$ in Lemma 4.4.1. Further define the following submodules of $A_{A}$ :

$$
A(i, j)=\left\langle z_{i}, W_{j}\right\rangle
$$

for $1 \leq i \leq n$ and $1 \leq j \leq(p-1) n$, and

$$
A(i, j, k)=\left\langle z_{i+1}+k w_{j+1}, A(i, j)\right\rangle
$$

for $1 \leq i \leq n-1,1 \leq j \leq(p-1) n-1$ and $1 \leq k \leq p-1$. Then a proper nonzero submodule of $A_{A}$ is one of the $A(i, j)$ or $A(i, j, k)$ for unique values of the parameters $i, j, k$.

Proof. Recall Remark 4.2.3. The $i$ th composition term (numbered in reverse order) of $Z$ is $A(i, 1)=\left\langle z_{i}\right\rangle$. Since $x$ acts trivially on $Z$, isomorphic sections of $Z$ and $W$ are either zero or composition factors, by Lemma 4.4.1. The Cartesian submodules of $Z / \operatorname{soc} A \oplus W / \operatorname{soc} A$ are the $A(i, j) / \operatorname{soc} A$. The composition factors of $Z / \operatorname{soc} A$ are the $\left\langle z_{i+1}+A(i, 1)\right\rangle / \operatorname{soc} A$ for $1 \leq i \leq n-1$, and the composition factors of $W / \operatorname{soc} A$ are the $\left\langle w_{j+1}+W_{j+1}\right\rangle / \operatorname{soc} A$ for $1 \leq j \leq(p-1) n-1$, by Lemma 4.4.1. Two simple $A$-modules are isomorphic by $\left|\operatorname{Aut}\left(C_{p}\right)\right|=p-1$ isomorphisms. By Theorem 4.2.1, 
the diagonal submodules of $Z / \operatorname{soc} A \oplus W / \operatorname{soc} A$ (those arising from isomorphisms between sections of composition length 1$)$ are the $A(i, j, k) / \operatorname{soc} A$.

Since $Z$ and $W$ are both uniserial, only Cartesian and restriction edges appear in the Hasse diagram of $\mathcal{L}(\operatorname{rad} A / \operatorname{soc} A)$. Therefore, pages of the type described in (v) and (vi) of Theorem 4.3.5 do not arise. The interval of definition of the diagonal vertex $A(i, j, k)$ is $\mathcal{L}(A(i+1, j+1) / A(i, j))$. Together with $\mathcal{L}(Z / \operatorname{soc} A) \times \mathcal{L}(W / \operatorname{soc} A)$, these pages constitute a complete and irredundant atlas of $\mathcal{L}(\operatorname{rad} A / \operatorname{soc} A)$. For $p=2$, the Hasse diagrams of pages in this atlas are illustrated in Figure 4.6.
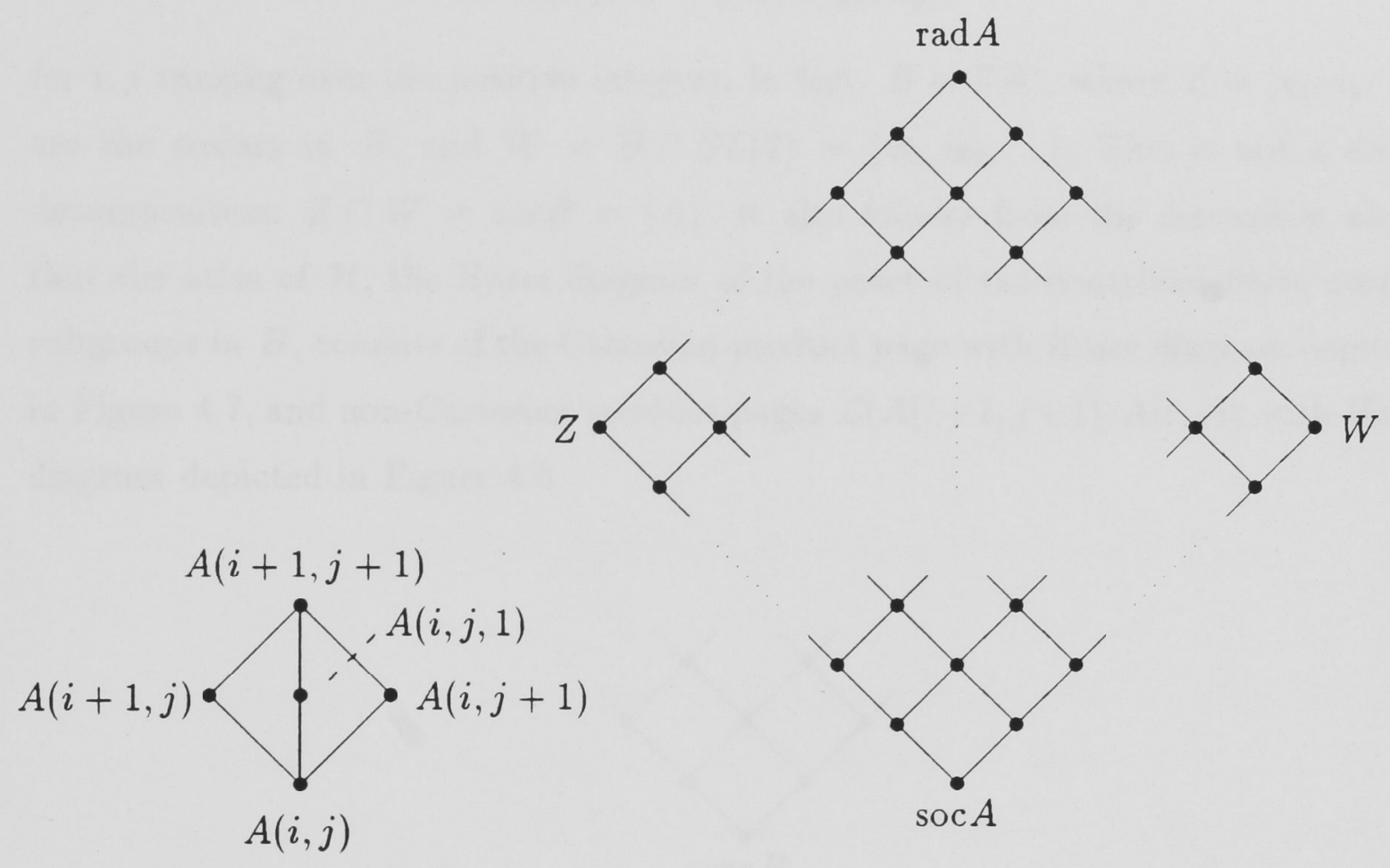

$$
\mathcal{L}(A(i+1, j+1) / A(i, j)) \quad \mathcal{L}(Z / \operatorname{soc} A) \times \mathcal{L}(W / \operatorname{soc} A)
$$

Figure 4.6: The atlas of the Hasse $\operatorname{diagram}$ of $\mathcal{L}(\operatorname{rad} A / \operatorname{soc} A)$ for $p=2$

Among other things, Conlon (1976) dealt with finite irreducible 2-subgroups of $G L(2)$. The Sylow 2-subgroups of $G L(2)$ are isomorphic to $C_{2 \infty}$ wr $C_{2}$. This infinite wreath product is the direct limit of the finite wreath products $C_{2^{n}}$ wr $C_{2}$, so that the finite normal subgroups of $C_{2 \infty}$ wr $C_{2}$ which lie in the base group $B$ correspond to normal subgroups in the base groups of these finite wreath products. In turn, the latter may be viewed as submodules of the $A_{A}$ discussed here (c.f. the discussion at the beginning of Section 2.1). In $G L(2), B$ appears as the group of diagonal 
matrices of 2 -power order. In this context, the generators $z_{i}$ and $w_{i}$ of the normal subgroups in $B$ may be defined as follows. For $k \geq 0$, let $\omega_{k}=\exp \left(2^{-k} \pi \sqrt{-1}\right)$, so that $\omega_{0}=-1, \omega_{k+1}^{2}=\omega_{k}$ and $\left\langle\omega_{0}, \omega_{1}, \ldots\right\rangle$ is the Sylow 2-subgroup $C_{2 \infty}$ of $C^{\times}$. Then $z_{i}=\left(\omega_{i-1}, \omega_{i-1}\right)$ and $w_{i}=\left(\omega_{i-1}, \omega_{i-1}^{-1}\right)$. For $p=2$ in Proposition 4.4.2, $(1-x)^{k}=2^{k-1}(1-x)$ and so $W$ as defined there is in fact a cyclic group of order $2^{n}$. Therefore, it follows from Proposition 4.4.2 that the nontrivial finite normal subgroups of $C_{2 \infty}$ wr $C_{2}$ lying in $B$ are the $A(i, j)$ and the $A(i, j, 1)$ defined by

$$
\begin{aligned}
& A(i, j)=\left\langle z_{i}, w_{j}\right\rangle \\
& A(i, j, 1)=\left\langle z_{i+1} w_{j+1}, w_{j}\right\rangle
\end{aligned}
$$

for $i, j$ ranging over the positive integers. In fact, $B=Z W$, where $Z=\left\langle z_{1}, z_{2}, \ldots\right\rangle$ are the scalars in $B$, and $W=B \cap S L(2)=\left\langle w_{1}, w_{2}, \ldots\right\rangle$. This is not a direct decomposition: $Z \cap W=\operatorname{soc} B=\left\langle z_{1}\right\rangle$. It also follows from the discussion above that the atlas of $\mathcal{H}$, the Hasse diagram of the poset of the nontrivial finite normal subgroups in $B$, consists of the Cartesian product page with Hasse diagram depicted in Figure 4.7, and non-Cartesian product pages $\mathcal{L}(A(i+1, j+1) / A(i, j))$ with Hasse diagram depicted in Figure 4.6.

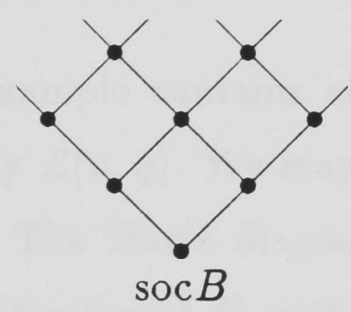

Figure 4.7: The Cartesian product page in the atlas of $\mathcal{H}$

This line of reasoning will be used in Section 5.2 in determining the poset of finite normal subgroups of $C_{2 \infty}$ wr $V_{4}$ which lie in the base group.

2. The abelian group $C_{4} \times C_{4}$ may be regarded as the direct sum of two isomorphic uniserial modules $U$ and $V$ of composition length 2 over the local ring $\mathbb{Z} / 4 \mathbb{Z}$. As in example 1 above, the Hasse diagram of $\mathcal{L}(U \oplus V)$ has no composition edges and the atlas of $\mathcal{L}(U \oplus V)$ has no pages of the type described in (v) and (vi) of Theorem 4.3.5. Let $U_{1}$ be the nonzero proper submodule of $U$ and $V_{1}$ its isomorphic image in $V$. There are two elements $[\alpha]$ and $[\beta]$ of $\mathcal{V}(U \oplus V)$ corresponding to the 
two isomorphisms of $U$ onto $V$. If underlining denotes restriction of domain from $U$ to $U_{1}$ and overlining restriction to $U / U_{1}$, then $[\underline{\alpha}]=[\underline{\beta}]$ is the unique diagonal vertex in the Hasse diagram of $\mathcal{L}\left(U_{1} \oplus V_{1}\right)$ and $[\bar{\alpha}]=[\bar{\beta}]$ is the unique diagonal vertex in the Hasse diagram of $\mathcal{L}\left(U \oplus V / U_{1} \oplus V_{1}\right)$. The Hasse diagram of $\mathcal{L}(U \oplus V)$ is given in Figure 4.8 .

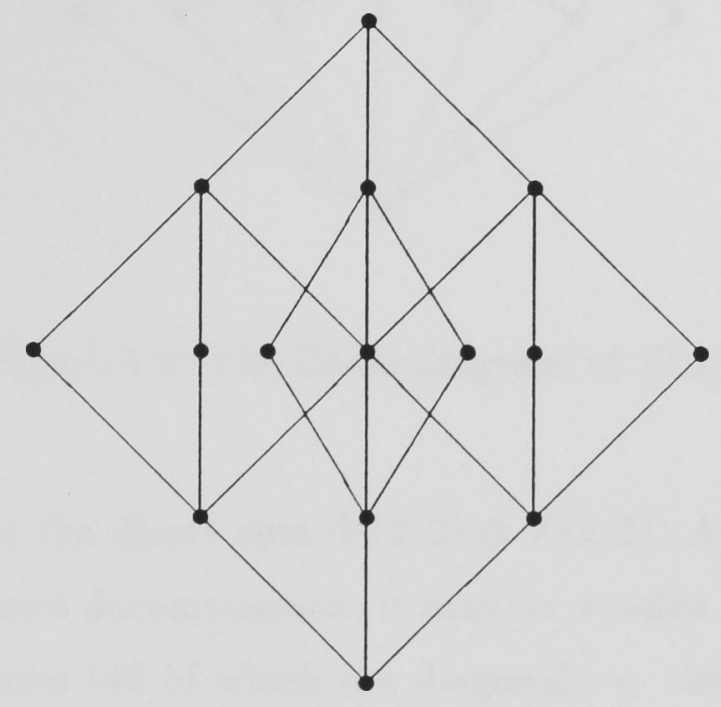

Figure 4.8: The Hasse diagram of the subgroup lattice of $C_{4} \times C_{4}$

3. The Hasse diagram in this example contains all three types of edges. Denote the subspace lattice of $V(n, q)$ by $\mathcal{L}(n, q)$. We may write $V(3,2)=U \oplus V$, where $U \cong V(2,2)$ and $V \cong V(1,2)$. The Hasse diagram of $\mathcal{L}(U) \times \mathcal{L}(V)$ is given in Figure 4.1. Obviously, a nonzero section of $U$ with an isomorphic image in $V$ has composition length 1 ; there are six such sections in $U$ and hence six diagonal vertices in the Hasse diagram of $\mathcal{L}(3,2)$. This gives us 16 vertices in all, agreeing with the count given by Gaussian coefficients. The only restriction edges are those occurring in the Hasse diagrams of the intervals of definition of the diagonal vertices: there are two of these for each diagonal vertex, and hence 12 restriction edges in all. Denote the three 1 -dimensional subspaces of $U$ as $U_{i}, 1 \leq i \leq 3$. There are six pairs of perspective edges in the Hasse diagram of $\mathcal{L}(U)$ : these are $\left(U / U_{i}, U_{j}\right)$ for $1 \leq i, j \leq 3$, $i \neq j$. Therefore, by Theorem 4.3.5, there are six composition edges in the Hasse diagram of $\mathcal{L}(3,2)$. Such an edge has one endpoint in $\mathcal{L}\left(U \oplus V / U_{j}\right)$ and the other endpoint in $\mathcal{L}\left(U_{i} \oplus V\right), i \neq j$. The total number of edges is $17+12+6=35$. The familiar Hasse diagram of $\mathcal{L}(3,2)$ is given in Figure 4.9. 


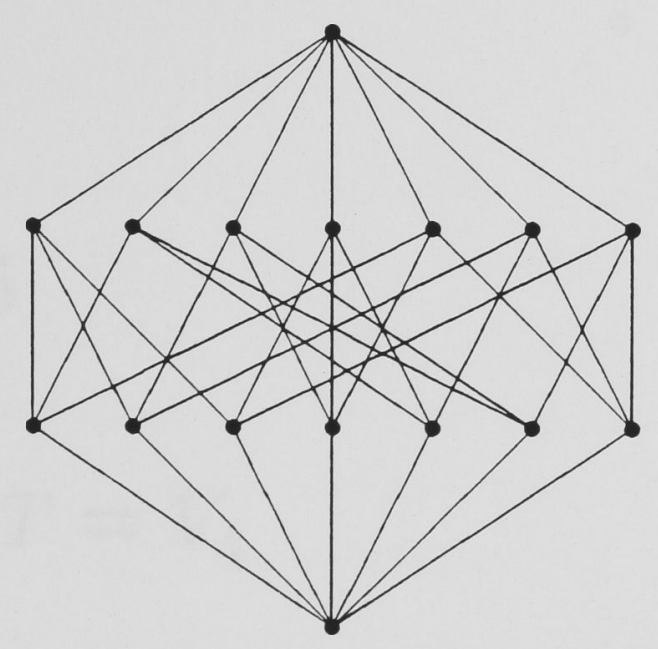

Figure 4.9: The Hasse diagram of $\mathcal{L}(3,2)$

4. Consider $V(4,2)$ as the direct sum $V(2,2) \oplus V(2,2)$. Applying the methods of this chapter to this direct decomposition, it may be verified that the Hasse diagram of $\mathcal{L}(4,2)$ has 67 vertices ( 42 of which are diagonal) - this agrees with the count given by Gaussian coefficients - and 240 edges (108 of which are restriction and 72 composition). There are six diagonal vertices corresponding to the six automorphisms of $V(2,2)$, and these are endpoints of 36 distinct edges. Every other edge appears in the Hasse diagram of a page isomorphic to $\mathcal{L}(3,2)$. The Hasse diagram of $\mathcal{L}(4,2)$ is too complicated to draw in full. The reduction to pages is not useful here - the preceding discussion shows that the whole lattice is a page in an irredundant atlas. Other methods may be more useful in situations such as this one. In Benson \& Conway (1985) for instance, a "diagram" for a general module $M$ is defined. This has as vertices the "one-headed" or "local" submodules; that is, the submodules with a unique maximal. For $R$ and $H$ as in Proposition 2.1.1, the local submodules of the $R H$-module $M$ are precisely its monogenic submodules. In this case, the local submodules join-generate $\mathcal{L}(M)$ (this is proved by induction on the composition length of $M$ ). The diagram for $M$ has both edges and broken lines (different from our edges and broken lines), and the Hasse diagram of $\mathcal{L}(M)$ may be recovered from it. For example, the diagram for $\mathcal{L}(n, q)$ is the projective space of $V(n, q)$. 


\section{Chapter 5}

\section{The case $T=V_{4}$}

In this chapter, we proceed with the programme outlined in Section 1.2, for the case $T=V_{4}$.

Recall the discussion at the beginning of Section 2.1. Denote by $A$ the group algebra $\left(\mathbf{Z} / 2^{n} \mathbf{Z}\right) V_{4}$. Each finite nonzero $V_{4}$-submodule of $B$ may also be viewed as a submodule of $A_{A}$ for sufficiently large $n$. To begin with, we consider the general problem of describing $\mathcal{L}\left(A_{A}\right)$. Using this description, we list finite $V_{4}$-submodules of $B$ and some of the inclusion relations between them. Then we solve the extension problem in $\mathrm{BS}_{4}$ for the faithful submodules, and, finally, sort the extensions so obtained into $G L(4)$-conjugacy classes.

Throughout, the generators (12)(34) and (13)(24) of $V_{4}$ are denoted by $a$ and $b$ respectively. The 4 -cycle (1234) in $D$ is denoted by $c$.

\subsection{Submodule properties of $A_{A}$}

In this section, we show how the theory of Chapter 4 may be used to describe $\mathcal{L}\left(A_{A}\right)$.

Recall that $\operatorname{soc} A$ is $\operatorname{simple}$ and $\operatorname{rad} A$ is maximal in $A_{A}$ (in particular, $A_{A}$ is indecomposable) and so each proper nonzero submodule of $A_{A}$ contains $\operatorname{soc} A$ and is contained in $\operatorname{rad} A$. Therefore, we need to describe $\mathcal{L}(\operatorname{rad} A / \operatorname{soc} A)$. It will be seen below that this problem may be reduced to describing the submodule lattices of certain sections of $\operatorname{rad} A / \operatorname{soc} A$.

For each $t \in V_{4} \backslash\{1\}$, denote by $F_{t}$ the submodule of $A_{A}$ generated by $2^{n-1}(1+t)$. In the next four results, we give some important properties of the $F_{t}$. We call a module cyclic if it is a cyclic group. A cyclic module is monogenic but of course the 
converse is not necessarily true.

Lemma 5.1.1 The noncyclic submodules of $A_{A}$ of order 4 are the $F_{t}$.

Proof. The set of isomorphism classes of noncyclic $A$-modules of order 4 is in oneto-one correspondence with the set of equivalence classes of representations of $V_{4}$ in $\operatorname{Aut}\left(C_{2} \times C_{2}\right) \cong S_{3}$. Therefore, by Proposition 2.1.3, the number of noncyclic submodules of $A_{A}$ of order 4 is at most the number of equivalence classes of representations of $V_{4}$ in $\operatorname{Aut}\left(C_{2} \times C_{2}\right)$. Let $\phi$ be such a representation. Clearly $\phi$ is not faithful. Also, $\operatorname{ker} \phi \neq V_{4}$, since $V_{4}$-trivial submodules of $A_{A}$ are cyclic. Thus $\operatorname{ker} \phi$ necessarily has index 2 . In this case, $\phi$ is determined by its kernel and image. The subgroups of $S_{3}$ of order 2 form a single conjugacy class and so there are precisely three equivalence classes of representations of $V_{4}$ in Aut $\left(C_{2} \times C_{2}\right)$, corresponding to the three subgroups of $V_{4}$ of order 2 .

We use Proposition 2.1.4 in the next few results.

Lemma 5.1.2 For involutions $t$ and $s$ of $V_{4}$ such that $t \neq s$, define

$$
F_{t}^{+}=\langle 1+t, s+s t\rangle / F_{t}
$$

and

$$
F_{t}^{-}=\langle 1-t, s-s t\rangle / F_{t}
$$

Then

$$
F_{t}^{\perp} / F_{t}=F_{t}^{+} \oplus F_{t}^{-}
$$

Also, $\mathcal{L}\left(F_{t}^{+}\right)$and $\mathcal{L}\left(F_{t}^{-}\right)$are isomorphic to the submodule lattice of the regular $\left(\mathbf{Z} / 2^{n-1} \mathbf{Z}\right) C_{2}$-module.

Proof. Certainly $F_{t}^{\perp}=\operatorname{ann}_{A} F_{t}$ contains $1+t$ and $1-t$. Since $F_{t}^{\perp} \cong A / F_{t}$ has order $2^{4 n-2}$, which is also the order of the $A$-submodule generated by $1+t$ and $1-t$, it follows that $F_{t}^{\perp}$ is generated as an $A$-module by $1+t$ and $1-t$ and the stated direct sum decomposition of $F_{t}^{\perp} / F_{t}$ is clear. Now $t$ acts as a scalar on both $F_{t}^{+}$and $F_{t}^{-}: t$ fixes the former and inverts the latter module. Therefore, the $A$-submodules of $F_{t}^{+}$and $F_{t}^{-}$are precisely their $\left(Z / 2^{n-1} \mathbf{Z}\right)\langle s\rangle$-submodules. As $s$-modules, $F_{t}^{+}$and $F_{t}^{-}$are isomorphic to the regular $\left(\mathbf{Z} / 2^{n-1} \mathbf{Z}\right)\langle s\rangle$-module.

The annihilator in $A$ of a cyclic submodule of $A_{A}$ will be called cocyclic. A submodule $M$ of $A_{A}$ is cocyclic if and only if $A_{A} / M$ is cyclic. 
Proposition 5.1.3 Let $M$ be a submodule of $A_{A}$. Then $M$ is neither cyclic nor cocyclic if and only if $F_{t} \leq M \leq F_{t}^{\perp}$ for some involution $t$ of $V_{4}$.

Proof. One direction is obvious. Conversely, suppose that $M$ is neither cyclic nor cocyclic. Then $M \geq F_{t}$ for some $t$ and also $M^{\perp} \geq F_{s}$ for some $s$. We will show that we may choose $t=s$.

Since $\Omega_{1}(A) \cong \mathrm{F}_{2} V_{4}$ has a unique maximal submodule by Proposition 2.1.2, $A_{A}$ has a unique elementary abelian submodule $E$ of order 8 . Therefore $E$ is generated by any two of the $F_{t}$ and for the rest of the proof we assume that $M \geq E$ and $M \not E^{\perp}$. This means that $\operatorname{rk}(M)=\operatorname{rk}\left(M^{\perp}\right)=2$ and thus $M \cong C_{2^{n}} \times C_{2^{n}}$.

Multiplication by $2^{n-1}$ is an endomorphism of each submodule of $A_{A}$, so that $A / 2 A \cong \Omega_{1}(A)$ and $(M+2 A) / 2 A \cong 2^{n-1} M=F_{t}$. For any submodule $U$ of order 4 in $\Omega_{1}(A)$, we have $U \cong \Omega_{1}(A) / U$. Therefore, as $A$-modules,

$$
F_{t} \cong \Omega_{1}(A) / F_{t} \cong A /(M+2 A) \cong(M+2 A)^{\perp}
$$

By Proposition 2.1.3, $F_{t}=(M+2 A)^{\perp}$. Hence $F_{t} \leq M^{\perp}$, verifying the claim.

The next result is obvious.

Lemma 5.1.4 Let $M$ be a submodule of $A_{A}$. Then $F_{t} \leq M \leq F_{t}^{\perp}$ for all $t$ if and only if $E \leq M \leq E^{\perp}$, where $E$ is the unique elementary abelian submodule of $A_{A}$ of order 8 .

Let $W_{1}$ and $W_{2}$ be submodules of $A_{A}$ and $M$ a cyclic maximal submodule of $W_{1}$. Either $W_{1} \cap W_{2}$ is cyclic, or $W_{2} \cap M$ is a cyclic maximal submodule of $W_{1} \cap W_{2}$. Therefore, the submodules of $A_{A}$ that are either cyclic or contain a cyclic maximal submodule form a meet-semilattice. We will denote this meet-semilattice by $\mathcal{C}$. Dually, we denote by $\mathcal{D}$ the join-semilattice consisting of submodules of $A_{A}$ that are either cocyclic or maximal in a cocyclic submodule.

Proposition 5.1.3 shows that the listing of the submodules of $A_{A}$ may be divided into two distinct parts. The first of these is listing, for each $t$, the submodules that contain $F_{t}$ and are contained in $F_{t}^{\perp}$. The second is listing the cyclic and cocyclic submodules. Likewise, listing the elements of $\mathcal{E}\left(A_{A}\right)$ may be divided (essentially) into two distinct parts. This is by way of the next result.

Proposition 5.1.5 If $W_{1} / W_{2} \in \mathcal{E}\left(A_{A}\right)$ then $W_{1} / W_{2}$ is an edge in the Hasse diagram of $\mathcal{C}, \mathcal{D}$, or $\mathcal{L}\left(F_{t}^{\perp} / F_{t}\right)$ for some $t \in V_{4} \backslash\{1\}$. 
Proof. If $W_{2}$ is cyclic (cocyclic), then $W_{1} \in \mathcal{C}\left(W_{1} \in \mathcal{D}\right)$. The previous statement is also true if " $W_{1}$ " and " $W_{2}$ " are interchanged. Suppose that $W_{1}$ and $W_{2}$ are neither cyclic nor cocyclic. By Proposition 5.1.3, $W_{1} \in \mathcal{L}\left(F_{t}^{\perp} / F_{t}\right)$ and $W_{2} \in \mathcal{L}\left(F_{s}^{\perp} / F_{s}\right)$ for some involutions $t$ and $s$ of $V_{4}$. If $t \neq s$ then $W_{2} \leq F_{t}^{\perp} \cap F_{s}^{\perp}=\left(F_{t}+F_{s}\right)^{\perp}=E^{\perp}$ and $W_{1} \geq F_{t}+F_{s}=E$. If $W_{2} \geq E$ then by Lemma 5.1 .4 we are done. Otherwise, $\left|W_{2}+E\right|=\left|W_{2}\right||E| / 4=\left|W_{1}\right|$, that is, $F_{s} \leq W_{1}=W_{2}+E \leq F_{s}^{\perp}$.

Note that, by duality, the Hasse diagram of $\mathcal{D}$ is known once that of $\mathcal{C}$ is known.

\subsection{Finite $V_{4}$-submodules of $B$}

The first result of this section says that each finite 2-subgroup of $B S_{4}$ with $V_{4}$ as projection group is conjugate to such a group whose diagonal subgroup is noncyclic. Thus, for the main purpose of listing conjugacy classes of irreducible groups, the cyclic $V_{\mathbf{4}}$-submodules of $B$ are not needed. In this section we concentrate on noncyclic $V_{4}$-submodules of $B$. (Cyclic submodules are used later on - see Proposition 5.4.11 - and it is more convenient to develop the required results there.)

Proposition 5.2.1 Let $G$ be a finite irreducible 2 -subgroup of $B S_{4}$ such that $B G=B V_{\mathbf{4}}$ and $B \cap G$ is cyclic. Then there is a $G L(4)$-conjugate of $G$ in $B V_{4}$ with noncyclic diagonal subgroup.

Proof. By Theorem 2 of Berger, Kovács \& Newman (1980), G is isomorphic to one (and only one) of the following groups of order $2^{k+3}$, for some $k \geq 2$ :

$$
\begin{aligned}
& D^{+}\left(2^{k+3}\right)=\left\langle d, e, f \mid d^{2^{k+1}}=e^{2}=f^{2}=1, d^{e}=d^{-1+2^{k}}, d^{f}=d^{1+2^{k}}, e f=f e\right\rangle \\
& Q^{+}\left(2^{k+3}\right)=\left\langle d, e, f \mid d^{2^{k+1}}=e^{2}=1, f^{2}=d^{2^{k}}, d^{e}=d^{-1+2^{k}}, d^{f}=d^{1+2^{k}}, e f=f e\right\rangle .
\end{aligned}
$$

Note that $\left\langle d^{2}, f\right\rangle$ is a noncyclic maximal abelian normal subgroup of both $D^{+}\left(2^{k+3}\right)$ and $Q^{+}\left(2^{k+3}\right)$ with quotient isomorphic to $V_{4}$. Hence, the result follows from Proposition 2.3.6.

It should be emphasised that we only need to list $V_{4}$-submodules of $B$ up to $S_{4}$ conjugacy. For if $G$ is a finite irreducible 2-subgroup of $B S_{4}$ such that $B G=B V_{4}$, then for any $s \in S_{4}, G^{s} \leq\left(B V_{4}\right)^{s}=B V_{4}$ is an extension of $B \cap G^{s}=(B \cap G)^{s}$ by $V_{4}$. By Proposition 5.2.1, then, our aim is to give a list of noncyclic finite $V_{4}$ submodules of $B$ containing at least one element from each $S_{4}$-conjugacy class of such submodules. 
We begin in the same way as the discussion following Figure 4.6. Each submodule in our list will be given by a group generating set. We now define the diagonal matrices which appear in these generating sets: let $\omega_{k}=\exp \left(2^{-k} \pi \sqrt{-1}\right)$ and

$$
\begin{aligned}
& x_{k}=\left(\omega_{k}, \omega_{k}, \omega_{k}, \omega_{k}\right), \\
& y_{k}=\left(\omega_{k}, \omega_{k}, \omega_{k}^{-1}, \omega_{k}^{-1}\right), \\
& u_{k}=\left(\omega_{k}, \omega_{k}^{-1}, \omega_{k}, \omega_{k}^{-1}\right), \\
& v_{k}=\left(\omega_{k}^{-1}, \omega_{k}, \omega_{k}, \omega_{k}^{-1}\right) .
\end{aligned}
$$

Note that $x_{0}=y_{0}=u_{0}=v_{0}=x_{1} y_{1} u_{1} v_{1}$, and denote by $B_{0}$ the subgroup generated by this element: it is the unique minimal $V_{4}$-submodule of $B$. Each automorphism of $V_{4}$ is realised as conjugation by an element of the subgroup $\langle(123),(12)\rangle \cong S_{3}$ of $S_{4}$. Notice that $u_{k}=y_{k}^{(23)}$ and $v_{k}=y_{k}^{(13)}$. Denote by $X$ the $V_{4}$-submodule $\left\langle x_{0}, x_{1}, \ldots\right\rangle$ of $B$ (the scalars of $G L(4)$ in $B$ ), by $Y$ the $V_{4}$-submodule $\left\langle y_{0}, y_{1}, \ldots\right\rangle$, and set $U=Y^{(23)}$ and $V=Y^{(13)}$. Note that $S_{3}$ permutes $Y, U$ and $V$. Furthermore, the $Y, U, V$ are respectively fixed elementwise by $a, b, a b$ and inverted by every other involution of $V_{4}$.

Clearly $B=X Y U V$. However, this is not a direct decomposition. The next lemma gives some of the intersections between products of the $X, Y, U, V$.

Lemma 5.2.2 Set $C_{1}=X, C_{a}=Y, C_{b}=U$ and $C_{a b}=V$. Then $B=\prod_{t \in V_{4}} C_{t}$ and $B \cap S L(4)=\prod_{t \in V_{\mathbf{4}} \backslash\{1\}} C_{t}$. Also, for distinct $t_{\boldsymbol{i}} \in V_{\mathbf{4}}$,

(i) $C_{t_{1}} \cap C_{t_{2}}=\Omega_{1}\left(C_{t_{1}}\right)=\Omega_{1}\left(C_{t_{2}}\right)=B_{0}$,

(ii) $C_{t_{1}} C_{t_{2}} \cap C_{t_{3}}=\Omega_{1}\left(C_{t_{3}}\right)=B_{0}$,

(iii) $C_{t_{1}} C_{t_{2}} \cap C_{t_{3}} C_{t_{4}}=\Omega_{1}\left(C_{t_{1}} C_{t_{2}}\right)=\Omega_{1}\left(C_{t_{3}} C_{t_{4}}\right)$

(iv) $C_{t_{1}} C_{t_{2}} C_{t_{3}} \cap C_{t_{4}}=\Omega_{2}\left(C_{t_{4}}\right)$

Proof. Certainly $Y U V \leq S L(4)$, and if $x z \in B \cap S L(4)$ for some $x \in X$ and $z \in Y U V$, then $x^{4}=1$. That is, $x \in\left\langle x_{1}\right\rangle$. Since $x_{1} y_{1} u_{1} v_{1} \in B_{0}$, we have that $\left\langle x_{1}\right\rangle \in Y U V$ and the second assertion follows. If $z \in C_{t_{1}} \cap C_{t_{2}}$, then, since $t_{1} \neq t_{2}$, $z$ is simultaneously fixed and inverted by an involution of $V_{4}$. Hence $z^{2}=1$ and $z \in \Omega_{1}\left(C_{t_{1}}\right)=\Omega_{1}\left(C_{t_{2}}\right)=B_{0}$. Similarly, either $C_{t_{1}} C_{t_{2}}$ or $C_{t_{3}}$ is fixed by an involution of $V_{4}$ and the other is inverted by that involution, and so (ii) is clear. By the same reasoning, (iii) follows. To prove (iv), it suffices to verify that $X \cap Y U V=\left\langle x_{1}\right\rangle$ 
and $Y \cap X U V=\left\langle y_{1}\right\rangle$, since $Y^{(23)}=U$ and $Y^{(13)}=V$. But this is obvious, since $x_{1} y_{1} u_{1} v_{1} \in B_{0}$.

The following notation is useful in describing conditions on parameters which we will use in labelling finite $V_{4}$-submodules of $B$.

Notation 5.2.3 For integers $i_{1}, \ldots, i_{m}$, write $i_{1}, \ldots, i_{m} \gtrsim 0$ to mean that either $i_{1}, \ldots, i_{m} \geq 1$ or $i_{1}=\cdots=i_{m}=0$.

In the sequel, when moving between the contexts of submodules of $B$ and submodules of the relevant $A_{A}$, we use implicitly the relevant one-to-one correspondence as defined at the beginning of Section 2.1.

By Lemma 5.1.1, the noncyclic $V_{4}$-submodules of $B$ of order 4 are (borrowing notation from Section 5.1):

$$
\begin{aligned}
& F_{a}=\left\langle x_{1} y_{1}, u_{1} v_{1}\right\rangle, \\
& F_{b}=\left\langle x_{1} u_{1}, y_{1} v_{1}\right\rangle, \\
& F_{a b}=\left\langle x_{1} v_{1}, y_{1} u_{1}\right\rangle .
\end{aligned}
$$

Since these submodules are $S_{4}$-conjugate and we intend to choose a single representative from each $S_{4}$-conjugacy class of finite $V_{4}$-submodules of $B$, for the rest of this section we restrict attention to submodules of $B$ which contain $F_{a}$.

By Lemma 5.2.2,

$$
B / F_{a}=\left(X Y / F_{a}\right) \times\left(U V / F_{a}\right)
$$

This direct decomposition of the $V_{4}$-module $B / F_{a}$ is the analogue of the direct decomposition of $F_{a}^{\perp} / F_{a}$ given in Lemma 5.1.2;XY/Fa and $U V / F_{a}$ match $F_{a}^{+}$and $F_{a}^{-}$respectively. Hence, by Lemma 5.1.2, Proposition 4.4.2 and the subsequent discussion in the first example of Section 4.4 , the finite $V_{4}$-submodules of $X Y$ properly containing $F_{a}$ are precisely those defined by

$$
F_{a}^{+}\left(i, j, \delta_{1}\right)=\left\langle x_{i}, y_{j},\left(x_{i+1} y_{j+1}\right)^{\delta_{1}}\right\rangle
$$

where $i, j \geq 1$ and $\delta_{1} \in\{0,1\}$. Note that $F_{a}$ itself may be included as $F_{a}=F_{a}^{+}(0,0,1)$. Similarly, the finite $V_{4}$-submodules of $U V$ properly containing $F_{a}$ are precisely the

$$
F_{a}^{-}\left(k, l, \delta_{2}\right)=\left\langle u_{k}, v_{l},\left(u_{k+1} v_{l+1}\right)^{\delta_{2}}\right\rangle
$$

where $k, l \geq 1$ and $\delta_{2} \in\{0,1\} ; F_{a}$ is included as $F_{a}^{-}(0,0,1)$. 
At the end of the first example in Section 4.4 (see Figures 4.6 and 4.7 ), we described essentially $\mathcal{E}\left(X Y / F_{a}\right)$ and $\mathcal{E}\left(U V / F_{a}\right)$. The first of these edge sets consists of the $F_{a}^{+}(i+1, j, 0) / F_{a}^{+}(i, j, 0), F_{a}^{+}(i, j, 1) / F_{a}^{+}(i, j, 0), F_{a}^{+}(i, j+1,0) / F_{a}^{+}(i, j, 0)$ and $F_{a}^{+}(i+1, j+1,0) / F_{a}^{+}(i, j, 1)$. The second edge set is described analogously, by replacing the superscript + with - .

We proceed by applying Theorem 4.3.5 to the direct decomposition (5.1) of $B / F_{a}$. For this we need to determine the $V_{\mathbf{4}}$-isomorphisms between the low sections of $X Y / F_{a}$ and $U V / F_{a}$.

There is, of course, precisely one isomorphism between any two zero sections. The submodule of $B$ corresponding to the isomorphism

$$
F_{a}^{+}\left(i, j, \delta_{1}\right) / F_{a}^{+}\left(i, j, \delta_{1}\right) \rightarrow F_{a}^{-}\left(k, l, \delta_{2}\right) / F_{a}^{-}\left(k, l, \delta_{2}\right)
$$

will be denoted by $F\left(i, j, k, l, \delta_{1}, \delta_{2}\right)$; it may be given in terms of group generators as

$$
F\left(i, j, k, l, \delta_{1}, \delta_{2}\right)=\left\langle x_{i}, y_{j}, u_{k}, v_{l},\left(x_{i+1} y_{j+1}\right)^{\delta_{1}},\left(u_{k+1} v_{l+1}\right)^{\delta_{2}}\right\rangle
$$

We regard these submodules as forming four families. In labels $F\left(i, j, k, l, \delta_{1}, \delta_{2}\right)$, the last two parameters, $\delta_{1}$ and $\delta_{2}$, range independently over $\{0,1\}$, and together they identify the family concerned. The first four parameters, $i, j, k, l$, indicate the position of the submodule within its family. The range of $i, j, k, l$ varies with $\delta_{1}, \delta_{2}$ : we have $i, j \geq 1$ when $\delta_{1}=0$ and $i, j \geq 0$ when $\delta_{1}=1$; similarly, $k, l \geq 1$ when $\delta_{2}=0$ and $k, l \gtrsim 0$ when $\delta_{2}=1$. To avoid overlong expressions, it will often be convenient to write $\delta$ for (the "vector" with "coordinates") $\delta_{1}, \delta_{2}$, and very informally we will use $F(i, j, k, l, \delta)$ as a generic label for members of these four families. These submodules are the Cartesian submodules with reference to the direct decomposition (5.1). In the present terms, the submodules of $X Y$ and $U V$ containing $F_{a}$ appear with new labels:

$$
\begin{aligned}
& F_{a}^{+}\left(i, j, \delta_{1}\right)=F\left(i, j, 0,0, \delta_{1}, 1\right) \\
& F_{a}^{-}\left(k, l, \delta_{2}\right)=F\left(0,0, k, l, 1, \delta_{2}\right)
\end{aligned}
$$

in particular, in this scheme $F_{a}$ itself has the label $F(0,0,0,0,1,1)$.

We now consider the diagonal submodules [with reference to (5.1)] corresponding to isomorphisms from simple (low) sections of $X Y / F_{a}$ to sections of $U V / F_{a}$. There is, of course, precisely one isomorphism between any pair of such sections. Given $i$ and $j$, there are three simple sections of the form $W / F_{a}^{+}(i, j, 0)$ in $X Y / F_{a}$, namely 
those with $W$ one of $F_{a}^{+}(i, j, 1), F_{a}^{+}(i, j+1,0)$, and $F_{a}^{+}(i+1, j, 0)$; we shall use $i, j$, and a parameter $\xi_{1}$ ranging over $\{-1,0,1\}$ in indexing these. (This will suit our description of the group generating sets for the relevant modules.) Similarly, given $k$ and $l$, there are three simple sections of the form $W / F_{a}^{-}(k, l, 0)$ in $U V / F_{a}$, namely those with $W$ one of $F_{a}^{-}(k, l, 1), F_{a}^{-}(k, l+1,0)$, and $F_{a}^{-}(k+1, l, 0)$, and we shall use a parameter $\xi_{2}$ ranging over $\{-1,0,1\}$ in indexing these. In both cases, the indexing is done according to the order of listing above. For example, the value of $\xi_{1}$ corresponding to the section $F_{a}^{+}(i, j, 1) / F_{a}^{+}(i, j, 0)$ is -1 . The submodule corresponding to the isomorphism from the simple section so indexed by $i, j, \xi_{1}$ to that indexed by $k, l, \xi_{2}$ will be called $F\left(i, j, k, l, 0,0, \xi_{1}, \xi_{2}\right)$; in terms of group generators,

$$
F\left(i, j, k, l, 0,0, \xi_{1}, \xi_{2}\right)=\left\langle x_{i}, y_{j}, u_{k}, v_{l}, x_{i+1}^{\xi_{1}} y_{j+1}^{\xi_{1}^{\prime}} u_{k+1}^{\xi_{2}} v_{l+1}^{\xi_{2}^{\prime}}\right\rangle
$$

where $\xi_{1}^{\prime}$ and $\xi_{2}^{\prime}$ denote the representatives in $\{-1,0,1\}$ of the congruence class modulo 3 of $1-\xi_{1}$ and $1-\xi_{2}$, respectively.

With $\xi_{1}$ and $\xi_{2}$ ranging independently over $\{-1,0,1\}$, these submodules form nine more families. The ranges of $i, j, k, l$ do not depend on $\xi_{1}$ and $\xi_{2}$ : given that here $\delta_{1}=\delta_{2}=0$, they range (independently) over the positive integers. To avoid overlong expressions, it will often be convenient to write $\xi$ for $\xi_{1}, \xi_{2}$, and the informal generic label for members of these nine families will be $F(i, j, k, l, 0,0, \xi)$.

There is only one other kind of simple (low) section in $X Y / F_{a}$ or in $U V / F_{a}$, namely the $F_{a}^{+}(i+1, j+1,0) / F_{a}^{+}(i, j, 1)$ and the $F_{a}^{-}(k+1, l+1,0) / F_{a}^{-}(k, l, 1)$. The submodule corresponding to the isomorphism from the first of these to the simple section indexed by $k, l, \xi_{2}$ above will be called $F\left(i, j, k, l, 1,0, \xi_{2}\right)$. The submodule corresponding to the isomorphism to the second of these from the simple section indexed by $i, j, \xi_{1}$ above will be called $F\left(i, j, k, l, 0,1, \xi_{1}\right)$. The submodule corresponding to the isomorphism $F_{a}^{+}(i+1, j+1,0) / F_{a}^{+}(i, j, 1) \rightarrow F_{a}^{-}(k+1, l+1,0) / F_{a}^{-}(k, l, 1)$ will be called $F(i, j, k, l, 1,1,1)$. In terms of group generators,

$$
\begin{aligned}
& F(i, j, k, l, 0,1, \xi)=\left\langle x_{i}, y_{j}, u_{k+1} v_{l+1}, v_{l}, x_{i+1}^{\xi} y_{j+1}^{\xi^{\prime}} u_{k+1}\right\rangle, \\
& F(i, j, k, l, 1,0, \xi)=\left\langle x_{i+1} y_{j+1}, y_{j}, u_{k}, v_{l}, x_{i+1} u_{k+1}^{\xi} v_{l+1}^{\xi^{\prime}}\right\rangle, \\
& F(i, j, k, l, 1,1,1)=\left\langle x_{i+1} y_{j+1}, y_{j}, u_{k+1} v_{l+1}, v_{l}, x_{i+1} u_{k+1}\right\rangle,
\end{aligned}
$$

where $\xi^{\prime}$ is the representative in $\{-1,0,1\}$ of the congruence class mod 3 of $1-\xi$. These modules form seven more families. The ranges of $i, j, k, l$ depend only on 
the $\delta$ involved.

We shall use $F(i, j, k, l, \delta, \xi)$ as a generic label for members of the twenty families of submodules identified so far, as follows. In the case of the four Cartesian families, $\xi$ stands for a void. In the case of the first nine diagonal families, $\delta$ is 0,0 and $\xi$ stands for $\xi_{1}, \xi_{2}$ as explained before. In the case of the next six diagonal families, $\delta$ is either 1,0 or 0,1 and $\xi$ stands for a single parameter ranging over $\{-1,0,1\}$; and in the case of the last family, $\delta, \xi$ is $1,1,1$. (These conventions are in line with the use of "A.B.C." as a generic name for a person without excluding people who have no middle name or more than one middle name.)

It remains to deal with submodules corresponding to isomorphisms between nonsimple sections. Since $a$ acts trivially on $X Y / F_{a}$ but invertingly on $U V / F_{a}$, isomorphic sections must have exponent 2 . In a module which as abelian group has rank 2 , the nonsimple elementary abelian (low) sections arise as the quotients of (finite) submodules over their multiples by the scalar 2: consequently, we know these sections as soon as we know the (finite) submodules. In particular, in $X Y / F_{a}$ the relevant sections are the $F_{a}^{+}\left(i+1, j+1, \delta_{1}\right) / F_{a}^{+}\left(i, j, \delta_{1}\right)$, and in $U V / F_{a}$ they are the $F_{a}^{-}\left(k+1, l+1, \delta_{2}\right) / F_{a}^{-}\left(k, l, \delta_{2}\right)$.

When $\delta_{1}$ or $\delta_{2}$ is 0 , the section is $V_{4}$-trivial. Thus, since $\left|\operatorname{Aut}\left(C_{2} \times C_{2}\right)\right|=6$, for each choice of $i, j, k, l$ there are six isomorphisms

$$
F_{a}^{+}(i+1, j+1,0) / F_{a}^{+}(i, j, 0) \rightarrow F_{a}^{-}(k+1, l+1,0) / F_{a}^{-}(k, l, 0) .
$$

Instead of using a $2 \times 2$ matrix over $F_{2}$ to describe such an isomorphism (which would mean using four more scalar parameters), we use two scalar parameters, $\alpha_{1}$ and $\alpha_{2}$, ranging over $\{-1,0,1\}$ subject to $\alpha_{1} \neq \alpha_{2}$. In effect, one of these represents the three choices for the first row of the $2 \times 2$ matrix, the other represents the choices for the second row; the two rows must be different. Then the corresponding submodules of $B$ are as follows:

$$
F\left(i, j, k, l, 0,0,2, \alpha_{1}, \alpha_{2}\right)=\left\langle x_{i}, y_{j}, u_{k}, v_{l}, x_{i+1} u_{k+1}^{\alpha_{1}} v_{l+1}^{\alpha_{1}^{\prime}}, y_{j+1} u_{k+1}^{\alpha_{2}} v_{l+1}^{\alpha_{2}^{\prime}}\right\rangle
$$

where $\alpha_{1}^{\prime}$ and $\alpha_{2}^{\prime}$ denote the representative in $\{-1,0,1\}$ of the congruence class modulo 3 of $1-\alpha_{1}$ and $1-\alpha_{2}$, respectively.

We regard these submodules as forming six families, according to the choices for the last two parameters. The informal generic label for the relevant submodules of $B$ is expanded to $F(i, j, k, l, \delta, \xi, \alpha)$. In the case of the twenty earlier families, 
$\alpha$ stands for a void while in the case of the six new families, $\xi$ stands for 2 (the common composition length of the domains of the relevant isomorphisms) and $\alpha$ stands for the pair $\alpha_{1}, \alpha_{2}$.

The nonsimple elementary abelian sections with $\delta_{1}=1$ or $\delta_{2}=1$ are not trivial but uniserial $V_{4}$-modules; they are not isomorphic to any section considered before but they are all isomorphic to each other. It is easy to see that, given $i, j, k, l$, there are precisely two $V_{4}$-isomorphisms

$$
F_{a}^{+}(i+1, j+1,1) / F_{a}^{+}(i, j, 1) \rightarrow F_{a}^{-}(k+1, l+1,1) / F_{a}^{-}(k, l, 1),
$$

and that the corresponding submodules of $B$ are as follows:

$$
F(i, j, k, l, 1,1,2, \alpha)=\left\langle x_{i}, y_{j}, u_{k}, v_{l}, x_{i+1} u_{k+1}, x_{i+2}^{\alpha} y_{j+2} u_{k+2} v_{l+2}\right\rangle,
$$

where $\alpha \in\{-1,1\}$. These submodules form two more families; once more, $\xi$ stands for 2. This gives us 28 families in all.

As a result of this application of Theorem 4.2.1, we have obtained a complete and irredundant list of those $V_{4}$-submodules of $B$ which contain $F_{a}$. For ease of reference, we give the following summary. The label of each submodule on the list starts with " $F\left(i, j, k, l, \delta_{1}, \delta_{2}\right.$ ", where $\delta_{1}$ and $\delta_{2}$ range independently over $\{0,1\}$ while $i, j, k, l$ range over the integers which satisfy the following conditions:

$i, j \geq 1$ or $i, j \geq 0$ according to whether $\delta_{1}$ is 0 or 1 , and

$k, l \geq 1$ or $k, l \gtrsim 0$ according to whether $\delta_{2}$ is 0 or 1 .

The label may then end with ")", in which case the submodule is Cartesian. Otherwise, the label continues subject to conditions which depend on the value of $\delta_{1}, \delta_{2}$. Namely,

when this parameter pair is 0,0 , the label may continue either with ", $\xi_{1}, \xi_{2}$ )" where $\xi_{1}, \xi_{2} \in\{-1,0,1\}$, or with ", $\left.2, \alpha_{1}, \alpha_{2}\right)$ " where $\alpha_{1}, \alpha_{2} \in\{-1,0,1\}$ and $\alpha_{1} \neq \alpha_{2}$;

when it is 0,1 or 1,0 , the label may continue with ", $\xi)$ " where $\xi \in\{-1,0,1\}$; and

when it is 1,1 , the label may continue either with ", 1)" or with ", $2, \alpha$ )" where $\alpha \in\{-1,1\}$.

In terms of subgroup generators, the descriptions of the modules on the list were given in (5.2)-(5.8); recall that in those, for parameters $\lambda$ ranging over $\{-1,0,1\}$, the convention is that $\lambda^{\prime}$ is the representative in that set of the congruence class of $1-\lambda$ modulo 3 . 
Theorem 5.2.4 The submodules defined in (5.2)-(5.8), with the parameter ranges as indicated, are pairwise distinct; they are precisely the $V_{4}$-submodules of $B$ containing $F_{a}$.

Next, we note how some properties of the listed modules can be read off from their labels. First,

$$
\begin{aligned}
& F(i, j, k, l, \delta, \xi, \alpha) \cap X Y=F_{a}^{+}\left(i, j, \delta_{1}\right), \\
& F(i, j, k, l, \delta, \xi, \alpha) \cap U V=F_{a}^{-}\left(k, l, \delta_{2}\right),
\end{aligned}
$$

whence

$$
\begin{aligned}
& F(i, j, k, l, \delta, \xi, \alpha) \cap X=\left\langle x_{i}\right\rangle, \\
& F(i, j, k, l, \delta, \xi, \alpha) \cap Y=\left\langle y_{j}\right\rangle, \\
& F(i, j, k, l, \delta, \xi, \alpha) \cap U=\left\langle u_{k}\right\rangle, \\
& F(i, j, k, l, \delta, \xi, \alpha) \cap V=\left\langle v_{l}\right\rangle .
\end{aligned}
$$

In particular, the bottom endpoint of the interval of definition of $F(i, j, k, l, \delta, \xi, \alpha)$ [that is, the unique maximal Cartesian submodule] is just $F(i, j, k, l, \delta)$. The top endpoint of the interval depends also on $\xi$. In order to determine the top endpoint, we refer to the discussion before Theorem 5.2.3, where we stated the domain and codomain of the isomorphism corresponding to the submodule. For example, since $F(i, j, k, l, 0,0,-1,-1)$ arises from the isomorphism

$$
F_{a}^{+}(i, j, 1) / F_{a}^{+}(i, j, 0) \rightarrow F_{a}^{-}(k, l, 1) / F_{a}^{-}(k, l, 0),
$$

it follows that the top endpoint of the corresponding interval is $F(i, j, k, l, 1,1)$. It should be clear that the top endpoint does not vary with $\alpha$. For example, given $i, j, k, l$, the six submodules of the form $F(i, j, k, l, 0,0,2, \alpha)$ have a common interval of definition, with top endpoint $F(i+1, j+1, k+1, l+1,0,0)$.

Now we turn to the problem of determining inclusion relations between the finite noncyclic $V_{4}$-submodules of $B$. By Proposition 5.1.5, the edges which concern us occur in the Hasse diagrams of the $\mathcal{L}\left(B / F_{t}\right)$. In fact, we consider $\mathcal{E}\left(B / F_{a}\right)$ only, since once this edge set is known, the other edge sets are known.

Recall the discussion at the end of Section 4.3. In the construction of an irredundant atlas, we only need to list those intervals of definition which are maximal. Observe that, except for $F_{a}^{+}(1,1,0) / F_{a}$, each simple section of $X Y / F_{a}$ is a section of a nonsimple section of the form $F_{a}^{+}(i+1, j+1,0) / F_{a}^{+}(i, j, 0)$; a similar statement holds for $U V / F_{a}$. It is easy to deduce from this that the maximal intervals of 
definition are precisely the following:

$$
\begin{aligned}
& F(i+1, j+1, k+1, l+1,1,1) / F(i, j, k, l, 1,1), \\
& F(i+1, j+1, k+1, l+1,0,0) / F(i, j, k, l, 0,0), \\
& F(1,1, k+1, l, 0,0) / F(0,0, k, l, 1,0), \\
& F(1,1, k, l+1,0,0) / F(0,0, k, l, 1,0), \\
& F(i+1, j, 1,1,0,0) / F(i, j, 1,1,0,1), \\
& F(i, j+1,1,1,0,0) / F(i, j, 1,1,0,1) .
\end{aligned}
$$

We are now ready to describe a complete and irredundant atlas of $\mathcal{L}\left(B / F_{a}\right)$.

Proposition 5.2.5 If $M / F_{a}$ is a maximal $V_{4}$-submodule in some finite $V_{4}$-submodule $N / F_{a}$ of $B / F_{a}$, then $N / M$ is represented by an edge in the Hasse diagram of at least one of the following submodule lattices:

$$
\begin{aligned}
& \mathcal{L}(F(i+1, j+1, k+1, l+1,0,0) / F(i, j, k, l, 0,0)) \cong \mathcal{L}(4,2), \\
& \mathcal{L}(F(i+1, j+1, k+1, l+1,1,1) / F(i, j, k, l, 1,1)) \cong \mathcal{L}\left(C_{4} \times C_{4}\right), \\
& \mathcal{L}(F(i+1, j+1,1,1,0,0) / F(i, j, 0,0,0,1)) \cong \mathcal{L}(3,2), \\
& \mathcal{L}(F(1,1, k+1, l+1,0,0) / F(0,0, k, l, 1,0)) \cong \mathcal{L}(3,2)
\end{aligned}
$$

Proof. We apply Theorem 4.3.5 to the direct decomposition (5.1). Figures 4.6 and 4.7 are useful here.

The maximal intervals of definition were listed above and some of these appear as pages of the form (5.9) and (5.10). We will determine pages of the types described in (v) and (vi) of Theorem 4.3.5, and it will be clear that those maximal intervals of definition which are not listed in the statement of the proposition are contained in such pages.

Denote by $\mathcal{S}$ the set of all low sections of $X Y / F_{a}$ that are isomorphic to sections of $U V / F_{a}$ (elements of $\mathcal{S}$ have composition length at most 2). In order to construct pages of the type described in $(\mathrm{v})$ of Theorem 4.3.5, we need to list the elements $W_{1} / W_{2} \in \mathcal{S}$ such that $W_{1}$ has a maximal submodule $M$ which does not contain $W_{2}$. If $W_{1} / W_{2}$ has length 2 then either $W_{2}=\operatorname{rad} W_{1}$ or $W_{1} / W_{2}$ is uniserial. In either case, each maximal submodule of $W_{1}$ contains $W_{2}$. The elements of $\mathcal{S}$ of length 1 satisfying the required conditions are of the form $F_{a}^{+}(i+1, j+1,0) / W_{2}$. If $M \nsupseteq W_{2}$ is maximal in $W_{1}$ then $M \cap W_{2}=F_{a}^{+}(i, j, 0)$. Now every length 1 section of $U V / F_{a}$ except $F_{a}^{-}(1,1,0) / F_{a}^{-}(0,0,1)$ is a section of $F_{a}^{-}(k+1, l+1,0) / F_{a}^{-}(k, l, 0)$ for some 
$k, l \geq 1$. Therefore, a page of type ( $\mathrm{v})$ is either of the form (5.11), or a subinterval of a page of the form (5.9). Similarly, a page of type (vi) is either a page of the form (5.12), or a subinterval of a page of the form (5.9). This completes the listing of the non-Cartesian product pages. It is clear that the atlas so constructed is complete and irredundant in the sense described at the end of Section 4.3.

Notice that pages of the form (5.11) and (5.12) are isomorphic as posets, but must be listed separately because of our labelling scheme.

The Hasse diagrams of the pages of the form (5.9)-(5.12) were discussed in Section 4.4. The full atlas given in Proposition 5.2.5 is not directly useful to us. We give only the Hasse diagrams of the pages $\mathcal{L}(F(i+1, j+1,1,1,0,0) / F(i, j, 0,0,0,1))$ and $\mathcal{L}(F(i+1, j+1,1,1,1,1) / F(i, j, 0,0,1,1))$, with labelled vertices, as these are relevant to Example 5.2.7 below. First, however, we need to determine which of the $V_{4}$-modules in the list of Theorem 5.2.4 are faithful (not all of them are), so that, by Theorem 2.3.5, we may recognise irreducible extensions of such modules.

Lemma 5.2.6 The non-faithful $V_{4}$-modules in the list of Theorem 5.2.4 are the $F(i, j, 0,0,0,1)$ and the $F(i, j, 0,0,1,1)$.

Proof. If $M \geq F_{a}$ is a finite $V_{4}$-submodule of $B$ then $C_{V_{4}}(M) \leq C_{V_{4}}\left(F_{a}\right)=\langle a\rangle$. Thus, if $M$ is not faithful then $C_{V_{4}}(M)=\langle a\rangle$ and so $M \leq X Y$; the finite submodules of $X Y$ containing $F_{a}$ have labels $F(i, j, 0,0,0,1)$ and $F(i, j, 0,0,1,1)$.

The following example is an application of Proposition 5.2.5 to a specific question which arises in the proof of Theorem 5.4.11.

Example 5.2.7 Suppose that $Q$ is a finite noncyclic $V_{4}$-submodule of $B$ and $C_{V_{4}}(Q)=\langle a\rangle$ (in particular, $Q \geq F_{a}$ is Cartesian). Which finite $V_{4}$-submodules of $B$ contain $Q$ as a maximal submodule? That is, which edges of the Hasse diagram of $\mathcal{L}\left(B / F_{a}\right)$ have bottom endpoint $Q$ ? We claim that such an edge occurs in the Hasse diagram of a page of the form (5.11), or (5.10) for $i=j=k=l=0$.

By Lemma 5.2.6, $Q=F(i, j, 0,0,0,1)$ for some $i, j \geq 1$, or $Q=F(i, j, 0,0,1,1)$ for some $i, j \gtrsim 0$. Since $Q$ is Cartesian, and the pages of Proposition 5.2.5 are just pairs of Cartesian submodules, it is easy to determine whether $Q$ is contained in a given page by referring to the Cartesian product subgraph. Certainly $Q$ is not in a page $(5.9)$ or $(5.12)$. In fact, $Q$ is in a page $(5.11)$, or $(5.10)$ for $k=l=0$. Refer to Figures 5.1 and 5.2. 


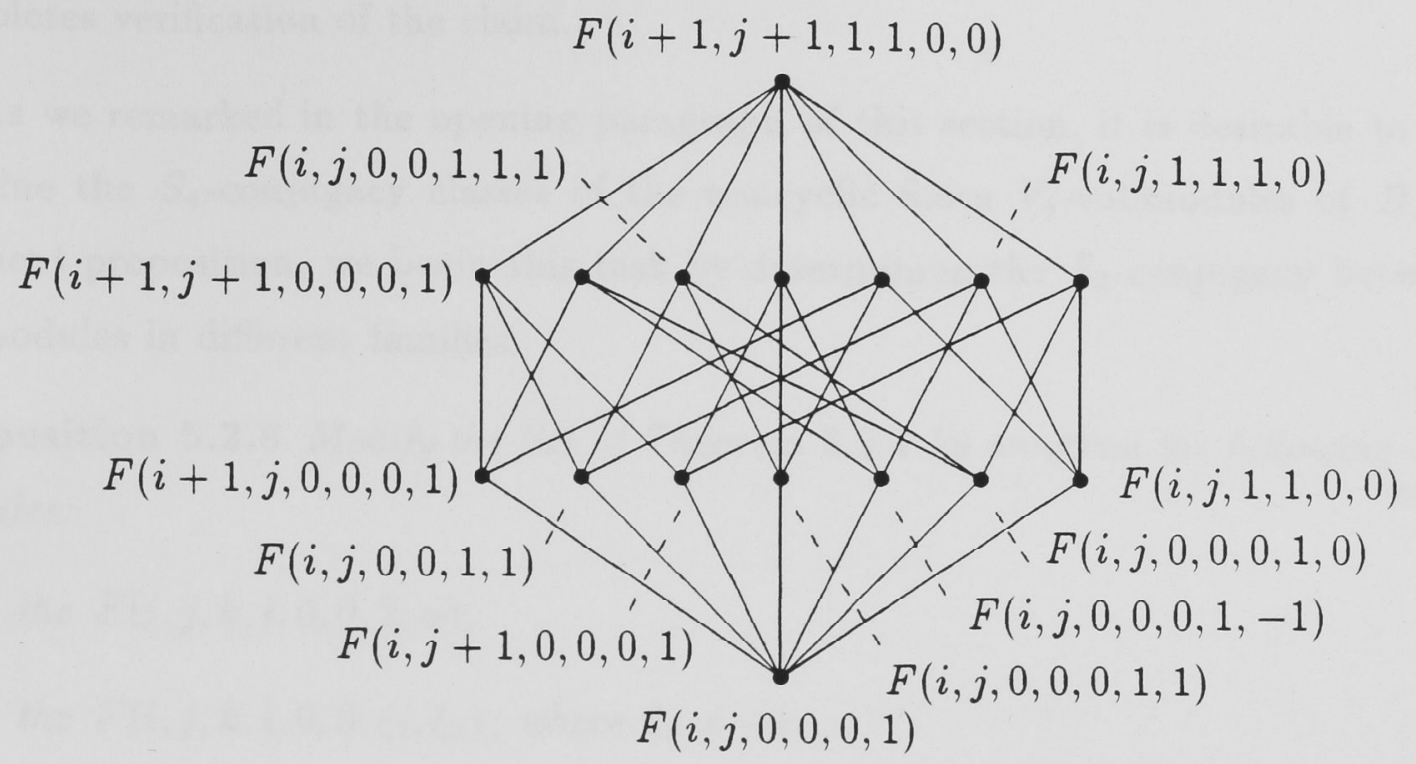

Figure 5.1: The Hasse diagram of $\mathcal{L}(F(i+1, j+1,1,1,0,0) / F(i, j, 0,0,0,1))$

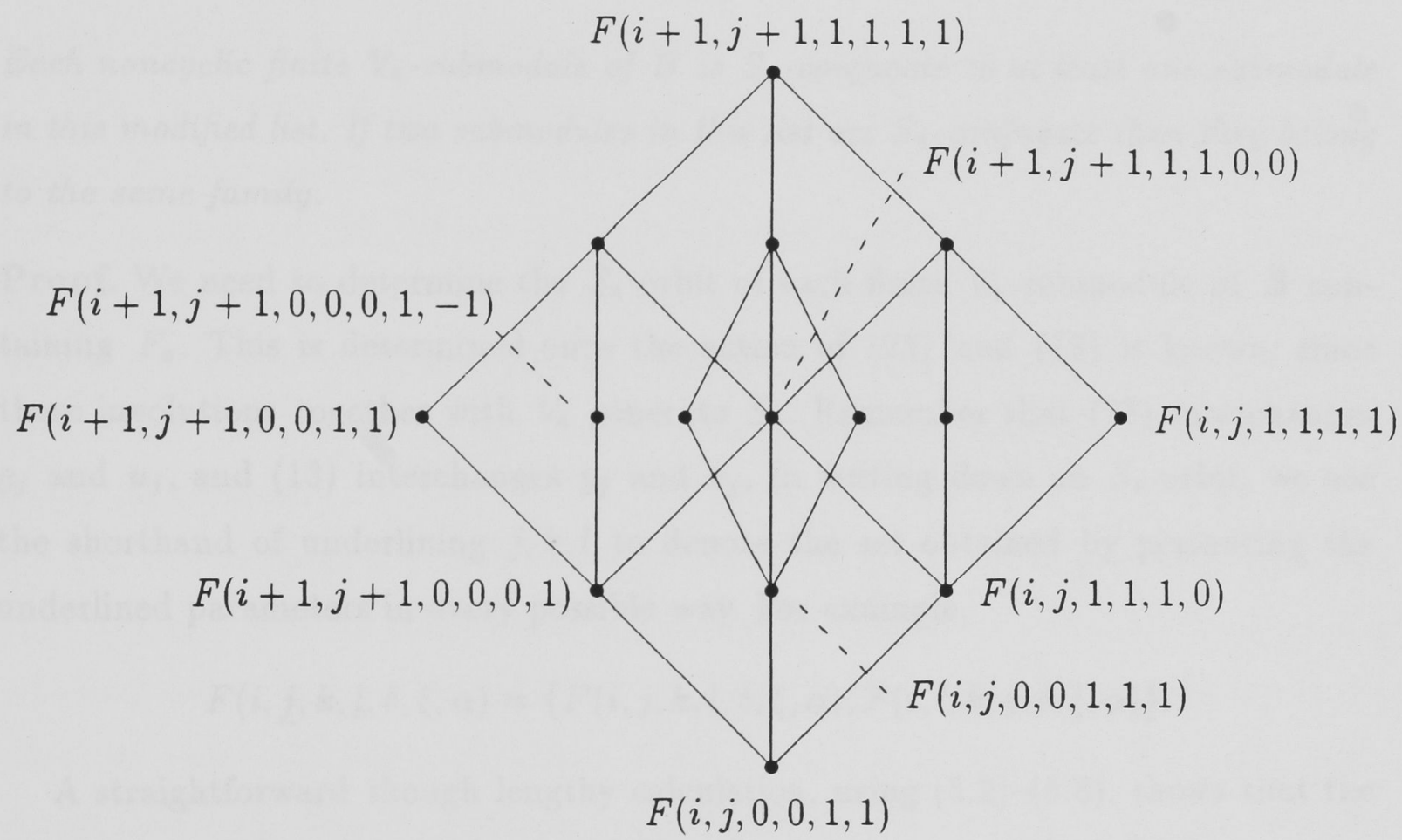

Figure 5.2: The Hasse diagram of $\mathcal{L}(F(i+1, j+1,1,1,1,1) / F(i, j, 0,0,1,1))$

Clearly $F(i, j, 0,0,1,1)$ is contained in a page (5.11) for all $i, j \geq 1$, but not for $i=j=0$ (the parameter conditions for the bottom endpoint $F(i, j, 0,0,0,1)$ of a page of this form are $i, j \geq 1)$. An edge with endpoint $F(i+1, j+1,0,0,0,1)$, $i, j \gtrsim 0$, in the Hasse diagram of a page (5.10), occurs in the Hasse diagram of a page (5.11): specifically, $\mathcal{L}(F(i+2, j+2,1,1,0,0) / F(i+1, j+1,0,0,0,1))$. This 
completes verification of the claim.

As we remarked in the opening paragraph of this section, it is desirable to determine the $S_{4}$-conjugacy classes of the noncyclic finite $V_{4}$-submodules of $B$. In the next proposition, we begin this task by determining the $S_{4}$-conjugacy between submodules in different families.

Proposition 5.2.8 Modify the list of Theorem 5.2.4 by omitting the following submodules:

- the $F(i, j, k, l, 0,0,2, \alpha)$;

- the $F\left(i, j, k, l, 0,0, \xi_{1}, \xi_{2}\right)$, where $\xi_{2} \neq-1$;

- the $F(i, j, k, l, 0,1,1)$, where $k, l \geq 1$;

- the $F(i, j, k, l, 1,0,0)$.

Each noncyclic finite $V_{4}$-submodule of $B$ is $S_{4}$-conjugate to at least one submodule in this modified list. If two submodules in this list are $S_{4}$-conjugate then they belong to the same family.

Proof. We need to determine the $S_{4}$-orbit of each finite $V_{4}$-submodule of $B$ containing $F_{a}$. This is determined once the action of (23) and (13) is known, since these involutions together with $V_{4}$ generate $S_{4}$. Remember that (23) interchanges $y_{j}$ and $u_{j}$, and (13) interchanges $y_{j}$ and $v_{j}$. In writing down an $S_{4}$-orbit, we use the shorthand of underlining $j, k, l$ to denote the set obtained by permuting the underlined parameters in every possible way. For example,

$$
F(i, \underline{j}, k, \underline{l}, \delta, \xi, \alpha)=\{F(i, j, k, l, \delta, \xi, \alpha), F(i, l, k, j, \delta, \xi, \alpha)\} .
$$

A straightforward though lengthy calculation, using (5.2)-(5.8), shows that the $S_{4}$-orbit of a submodule in the list of Theorem 5.2.4 is one of the following:

$$
\begin{aligned}
& F(i, j, \underline{k, l}, 0,1) \cup F(i, \underline{k}, j, \underline{l}, 0,0,0,0) \cup F(i, \underline{l, k}, j, 0,0,0,1) ; \\
& F(i, j, \underline{k, l}, 1,0) \cup F(i, \underline{k}, j, \underline{l}, 0,0,1,1) \cup F(i, \underline{l, k}, j, 0,0,1,0) ; \\
& F(i, j, \underline{k, l}, 1,1) \cup F(i, \underline{k}, j, \underline{l}, 0,0,2,1,0) \cup F(i, \underline{l}, k, j, 0,0,2,0,1) ; \\
& F(i, j, \underline{k, l}, 0,0,1,-1) \cup F(i, \underline{k}, j, \underline{l}, 0,0,-1,0) \cup F(i, \underline{l, k}, j, 0,0,-1,1) ; \\
& F(i, j, \underline{k, l}, 0,1,-1) \cup F(i, \underline{k}, j, \underline{l}, 0,0,2,-1,0) \cup F(i, \underline{l, k}, j, 0,0,2,-1,1) ; \\
& F(i, \underline{j, k}, l, 1,0,1) \cup F(i, \underline{j}, l, \underline{k}, 1,0,0) \cup F(i, l, \underline{k, j}, 0,1,1) ; \\
& F(i, j, \underline{k, l}, 1,0,-1) \cup F(i, \underline{k}, j, \underline{l}, 0,0,2,1,-1) \cup F(i, \underline{l, k}, j, 0,0,2,0,-1) ;
\end{aligned}
$$




$$
\begin{aligned}
& F(i, \underline{j, k, l}, 0,0) ; \\
& F(i, \underline{j, k, l}, 0,0,-1,-1) ; \\
& F(i, \underline{j, k, l}, 0,0,0,-1) ; \\
& F(i, \underline{j, k, l}, 0,1,0) ; \\
& F(i, \underline{j, k, l}, 1,1,1) ; \\
& F(i, \underline{j, k, l}, 1,1,2, \alpha) .
\end{aligned}
$$

It is apparent that the values of parameters in some of these labels violate the conditions set down before Theorem 5.2.4. For example, in the first line, $i=j=1$, $k=l=0$ is admissible only in the first join component. Although a label may be "inadmissible" in this sense, it still describes a finite $V_{\mathbf{4}}$-submodule of $B$ in terms of group generators, by the relevant one of the definitions (5.2)-(5.8). At this stage, all we can say is that a submodule so described by an inadmissible label either does not contain $F_{a}$, or it does contain $F_{a}$ but occurs in the list of Theorem 5.2.4 with another label. In fact, we shall see that the second situation does not occur.

The first claim of the proposition follows from inspection of the orbits listed above. Namely, apart from the $F(0,0, k, l, 1,0,0)$, the labels of all the modules proposed for omission have all four initial parameters positive, so in their orbits inadmissible labels do not arise at all, and we see that each of them is $S_{4}$-conjugate to some submodule which is not proposed for omission. We also see that $F(0,0, k, l, 1,0,0)$ is $S_{4}$-conjugate to $F(0,0, l, k, 1,0,1)$, the latter label being admissible and not proposed for omission.

The second claim is equally straightforward for all the cases involving only positive $i, j, k, l$. For the submodules $M$ with $i=j=0$ or $k=l=0$ which are retained, one calculates the additive rank and finds it to be almost always 2 . In all those cases, $\Omega_{1}(M)=F_{a}$. Thus, if for some $r \in S_{4}$ we have $M^{r} \geq F_{a}$, then $r$ normalises $F_{a}$, that is, centralises $a$. The centraliser of $a$ in $S_{4}$ is generated by $V_{4}$ and (12). Now (12) centralises $X Y$ and interchanges $u_{k}$ and $v_{k}$, so that it is straightforward to check case by case that the second claim holds for all submodules of rank 2 . The only $M$ of larger rank are the $F(0,0,0,0,1,1,2, \alpha)$, and these are simply normalised by $S_{4}$.

Remark 5.2.9 From the proof of Proposition 5.2.8 we reiterate the fact that if a submodule appears in the list of $S_{4}$-orbits above with an inadmissible label then that submodule does not contain $F_{a}$, and thus also does not occur in the list of 
Theorem 5.2.4 with any other label.

\subsection{The extension problem}

In this section, we determine the $B V_{4}$-conjugacy classes of $V_{4}$-extensions in $B S_{4}$ of the modules listed in Theorem 5.2.4. Theorem 5.3.3 shows that the first step in this determination is to calculate the orders of certain second cohomology groups of $V_{4}$. To do this, we use the theory of Chapter 3, particularly Section 3.4.

Lemma 5.3.1 Let $T$ be a transitive 2 -subgroup of $S_{4}$. If $|T|=4$ then $H^{i}(T, B)$ is zero for all $i \geq 1$. If $|T|=8$ then $H^{2 i}(T, B)=0$ and $H^{2 i-1}(T, B)=C_{2}$, for all $i \geq 1$.

Proof. If $T$ has order 4 , then $B=\operatorname{Ind}_{1}^{T} C_{2 \infty}$. If $T$ has order 8 , then $B=\operatorname{Ind}_{\langle t\rangle}^{T} C_{2 \infty}$, where $t$ is a transposition in $T$ and $C_{2 \infty}$ is trivial under the action of $t$. The lemma follows from Propositions 3.1.7 and 3.1.8.

Lemma 5.3.2 Define a map $\delta: D \rightarrow B$ by $a c^{i} \delta=x_{0}$ and $c^{i} \delta=0,1 \leq i \leq 4$. Then $\delta$ is a non-inner derivation from $D$ to $B$.

Proof. Clearly, $\delta$ is a derivation from $\langle a\rangle$ to $B$ and from $\langle c\rangle$ to $B$. Thus $\delta$ is a derivation from $D$ to $B$ by Lemma 3.4.4. If $\delta$ were an inner derivation, say $g \delta=w(1-g)$ for all $g \in D$ and some $w \in U$, then we would have $w(1-c)=0$. That is, $w \in X$ and $a \delta=0$, a contradiction.

Theorem 5.3.3 Let $T$ be a transitive 2-subgroup of $S_{4}$ and $B_{1}$ a finite $T$ submodule of $B$ with more than one element. Then $\left|H^{2}\left(T, B_{1}\right)\right|$ is the number of $B T$-conjugacy classes of finite subgroups $G$ of $B S_{4}$ such that $B \cap G=B_{1}$ and $B G=B T$.

Proof. Clearly, the number of $B T$-conjugacy classes of finite 2 -subgroups $G$ of $B S_{4}$ such that $B \cap G=B_{1}$ and $B G=B T$ is the same as the number of conjugacy classes of complements of $B / B_{1}$ in $B T / B_{1} \cong B / B_{1} \rtimes T$. This number, of course, is $\left|H^{1}\left(T, B / B_{1}\right)\right|$.

Consider the short exact sequence

$$
0 \rightarrow B_{1} \rightarrow B \rightarrow B / B_{1} \rightarrow 0
$$


By Proposition 3.1.1, we have a long exact sequence

$$
\cdots \rightarrow H^{1}(T, B) \stackrel{\gamma}{\rightarrow} H^{1}\left(T, B / B_{1}\right) \rightarrow H^{2}\left(T, B_{1}\right) \rightarrow H^{2}(T, B) \rightarrow \cdots,
$$

where $\gamma$ is induced by the natural surjection of $B$ onto $B / B_{1}$. If $|T|=4$ then the result follows from Lemma 5.3.1. If $T=D$ then $H^{2}(T, B)=0$ and $H^{1}(T, B)=C_{2}$ by Lemma 5.3.1. Thus, $\gamma$ is zero or an embedding. The derivation $\delta$ from $D$ to $B$ defined in Lemma 5.3.2 is a representative of the unique nonzero element of $H^{1}(D, B)$. Since $x_{0} \in B_{1},[\delta] \gamma=0$. Thus $H^{1}\left(T, B / B_{1}\right) \cong H^{2}\left(T, B_{1}\right)$.

Suppose that we are given a (noncyclic) finite $V_{4}$-submodule $B_{1}$ of $B$. Our aim in this section is to construct a complete list of $B V_{4}$-conjugacy class representatives of supplements $G$ of $B$ in $B V_{4}$ such that $B \cap G=B_{1}$. The first step is to calculate $\left|H^{2}\left(V_{4}, B_{1}\right)\right|$. The second is to construct a list of such supplements which contains at least one group from each $B V_{4}$-conjugacy class. If this list contains precisely $\left|H^{2}\left(V_{4}, B_{1}\right)\right|$ groups, then, by Theorem 5.3.1, no two members of the list can be $B V_{4}$ conjugate, so the list is of the kind that we sought. In later sections we determine which of the representatives in the list are conjugate by other elements of $G L(4)$.

Now we begin the calculation of $\left|H^{2}\left(V_{4}, B_{1}\right)\right|$. In the notation of Section 3.4, $G=V_{\mathbf{4}}, N=\langle a\rangle$ and $y=b$. Since $x^{y}=x$, the action $\circ$ of $\langle b\rangle$ on $H^{1}\left(N, B_{1}\right)$ given in Lemma 3.4 .1 is induced by the action of $b$ on $B_{1}$.

Lemma 5.2.2 and the following are used repeatedly.

Lemma 5.3.4 Let $t$ and $s$ be distinct involutions of $V_{4}$. Suppose that $C_{t}$ and $C_{s}$ are cyclic or quasicyclic $V_{4}$-submodules of $B$ such that $C_{t}$ is fixed by $t$ and $C_{s}$ is fixed by $s$ and inverted by $t$. Then

$$
\left(B_{1} \cap C_{t} C_{s}\right)^{1-t}=\left\{\begin{array}{cl}
\left(B_{1} \cap C_{s}\right)^{2} & \text { if } B_{1} \cap C_{t} C_{s}=\left(B_{1} \cap C_{t}\right)\left(B_{1} \cap C_{s}\right) \\
B_{1} \cap C_{s} & \text { otherwise }
\end{array}\right.
$$

and

$$
\left(B_{1} \cap C_{t} C_{s}\right)^{1+t}=\left\{\begin{array}{cl}
\left(B_{1} \cap C_{t}\right)^{2} & \text { if } B_{1} \cap C_{t} C_{s}=\left(B_{1} \cap C_{t}\right)\left(B_{1} \cap C_{s}\right) \\
B_{1} \cap C_{t} & \text { otherwise. }
\end{array}\right.
$$

Proof. First note that

$$
\begin{aligned}
\left(B_{1} \cap C_{s}\right)^{2} & =\left(B_{1} \cap C_{t}\right)^{1-t} \cdot\left(B_{1} \cap C_{s}\right)^{1-t} \\
& \leq\left(B_{1} \cap C_{t} C_{s}\right)^{1-t} \\
& \leq B_{1} \cap C_{s} .
\end{aligned}
$$


Since $\left|B_{1} \cap C_{s}:\left(B_{1} \cap C_{s}\right)^{2}\right|=2,\left(B_{1} \cap C_{t} C_{s}\right)^{1-t}$ is either $B_{1} \cap C_{s}$ or $\left(B_{1} \cap C_{s}\right)^{2}$ The $V_{4}$-endomorphism $1-t$ of $B$ has kernel $B_{1} \cap C_{t}$ on both $B_{1} \cap C_{t} C_{s}$ and $\left(B_{1} \cap C_{t}\right)\left(B_{1} \cap C_{s}\right)$, since $\Omega_{1}\left(C_{t}\right)=\Omega_{1}\left(C_{s}\right)=B_{0} \leq B_{1}$. Furthermore, $1-t$ has image $\left(B_{1} \cap C_{s}\right)^{2}$ on $\left(B_{1} \cap C_{t}\right)\left(B_{1} \cap C_{s}\right)$, and the first two assertions are clear. The other assertions are proved similarly.

Lemma 5.3.4 is typical of the comparisons between subgroups of $B_{1}$ that we must make in subsequent calculations: how products of $X, Y, U$ and $V$ intersect $B_{1}$. The generating sets given in Theorem 5.2.4 allow us to make these comparisons quite easily. For on $B_{1}$, the $V_{4}$-endomorphism $1-t$ has kernel $B_{1} \cap X C_{t}$ (in the notation of Lemma 5.2.2) and $1+t$ has kernel $B_{1} \cap C_{s} C_{s^{\prime}}$, where $s, s^{\prime}, t \in V_{4} \backslash\{1\}$ and $s, s^{\prime}$ are distinct from $t$. It should be noted that $\left(B_{1} \cap X\right)\left(B_{1} \cap Y\right)$ has index at most 2 in $B_{1} \cap X Y$. After conjugation by the appropriate element of $S_{4}$, we may replace $Y$ by $C_{t}$ in the previous statement. A quick calculation using Lemmas 5.2.2 and 5.3.4 then shows that

$$
H^{i}\left(\langle b\rangle, B_{1}^{N}\right)=\left\{\begin{array}{cl}
C_{2} & \text { if } B_{1} \cap X Y=\left(B_{1} \cap X\right)\left(B_{1} \cap Y\right) \\
0 & \text { otherwise. }
\end{array}\right.
$$

The differentials $d_{2}^{0,1}$ and $d_{2}^{1,1}$ have codomains $H^{2}\left(\langle a\rangle, B_{1}^{N}\right)$ and $H^{3}\left(\langle a\rangle, B_{1}^{N}\right)$ respectively.

Lemma 5.3.5 If $B_{1} \cap X Y \neq\left(B_{1} \cap X\right)\left(B_{1} \cap Y\right)$ or $B_{1} \cap U V=\left(B_{1} \cap U\right)\left(B_{1} \cap V\right)$ then $d_{2}^{0,1}=0$. Otherwise, $d_{2}^{0,1} \neq 0$ if and only if $B_{1} \cap X Y V \neq\left(B_{1} \cap X\right)\left(B_{1} \cap Y V\right)$, in which case $\operatorname{im} d_{2}^{0,1}=H^{2}\left(\langle b\rangle, B_{1}^{N}\right) \cong C_{2}$.

Proof. This is an application of Theorem 3.4.5. If $B_{1} \cap X Y \neq\left(B_{1} \cap X\right)\left(B_{1} \cap Y\right)$ then, by $(5.13), d_{2}^{0,1}=0$.

Let $r B_{1}^{1-a}$ be a $b$-fixed element of $H^{1}\left(\langle a\rangle, B_{1}\right)=B_{1} \cap U V / B_{1}^{1-a}$, and choose $q \in B_{1}$ such that

$$
q^{1-a}=r^{1-b}
$$

Then

$$
\left(r B_{1}^{1-a}\right) d_{2}^{0,1}=q^{1+b}\left(B_{1} \cap X Y\right)^{1+b} .
$$

If $r \in\left(B_{1} \cap U\right)\left(B_{1} \cap V\right)$ then by Lemma 5.3.4 we may choose $q \in B_{1} \cap V$ : in this case $q^{1+b}=0$ and hence $\left(r B_{1}^{1-a}\right) d_{2}^{0,1}=0$. Otherwise, $B_{1} \cap U V \neq\left(B_{1} \cap U\right)\left(B_{1} \cap V\right)$. That is, we may choose $r=u v$, where $u \in U, v \in V$ and $u, v \notin B_{1}$. Then 
$r^{1-b}=v^{2}=v^{1-a}=q^{1-a}$, whence $q=x y v$ for some $x \in X, y \in Y$, and $q^{1+b}=x^{2}$. If $d_{2}^{0,1} \neq 0$ then for some such choice of $r$ and $q$, necessarily $x \notin B_{1}$. That is, by Lemma 5.2.2, $B_{1} \cap X Y V \neq\left(B_{1} \cap X\right)\left(B_{1} \cap Y V\right)$.

The next lemma is an application of Proposition 3.4.10. Its proof is similar to that of Lemma 5.3.5 and so is omitted.

Lemma 5.3.6 If $B_{1} \cap X Y \neq\left(B_{1} \cap X\right)\left(B_{1} \cap Y\right)$ or $B_{1} \cap U V=\left(B_{1} \cap U\right)\left(B_{1} \cap V\right)$ then $d_{2}^{1,1}=0$. Otherwise, $d_{2}^{1,1} \neq 0$ if and only if $B_{1} \cap X Y U \neq\left(B_{1} \cap X U\right)\left(B_{1} \cap Y\right)$, in which case $\operatorname{im} d_{2}^{1,1}=H^{3}\left(\langle b\rangle, B_{1}^{N}\right) \cong C_{2}$.

The differentials $d_{2}^{0,2}$ and $d_{3}^{0,2}$ have domains in $H^{2}\left(\langle a\rangle, B_{1}\right)=B_{1} \cap X Y / B_{1}^{1+a}$. For each $r \in B_{1} \cap X Y$, define a 2 -cocycle $\psi_{r}$ from $N$ to $B_{1}$ by $(a, a) \psi_{r}=r$. The map

$$
r B_{1}^{1+a} \mapsto\left[\psi_{r}\right]
$$

is an isomorphism of $B_{1} \cap X Y / B_{1}^{1+a}$ onto $H^{2}\left(N, B_{1}\right)$, with inverse

$$
[\psi] \mapsto(a, a) \psi B_{1}^{1+a} .
$$

The action $\circ$ of $b$ on $H^{2}\left(\langle a\rangle, B_{1}\right)$ given in Lemma 3.4.3 is induced by the action of $b$ on $B_{1}$.

Lemma 5.3.7 Suppose that $r B_{1}^{1+a}$ is a $b$-fixed element of $B_{1} \cap X Y / B_{1}^{1+a}$, and choose $q_{1} \in B_{1}$ such that

$$
r^{1-b}=q_{1}^{1+a} .
$$

Then $\left(r B_{1}^{1+a}\right) d_{2}^{0,2}=0$ if and only if there exists $q_{2} \in B_{1}$ such that

$$
q_{1}^{1+b}=q_{2}^{1-a} .
$$

Proof. This is just a restatement of Proposition 3.4.7, using the isomorphism defined in the preceding discussion.

The next two results are obtained by reasoning similar to that used in the proof of Lemma 5.3.5. The first follows from Lemma 5.3.7, the second from Corollary 5.3.8 and Proposition 3.4.11.

Corollary 5.3.8 If $B_{1} \cap X Y=\left(B_{1} \cap X\right)\left(B_{1} \cap Y\right)$ or $B_{1} \cap Y U V=\left(B_{1} \cap Y\right)\left(B_{1} \cap U V\right)$ then $d_{2}^{0,2}=0$. Otherwise, $d_{2}^{0,2} \neq 0$ if and only if $B_{1} \cap X Y U=\left(B_{1} \cap X Y\right)\left(B_{1} \cap U\right)$ and $B_{1} \cap Y U V \neq\left(B_{1} \cap Y V\right)\left(B_{1} \cap U\right)$. 
Lemma 5.3.9 $d_{3}^{0,2}=0$.

By Lemma 5.3.9 and (3.10),

$$
\left|H^{2}\left(V_{4}, B_{1}\right)\right|=\left|\operatorname{coker} d_{2}^{0,1}\right|\left|\operatorname{ker} d_{2}^{1,1}\right|\left|\operatorname{ker} d_{2}^{0,2}\right| .
$$

From now on, we are interested only in the case that $B_{1} \geq F_{a}$. The form of the group generators of such submodules $B_{1}$ of $B$ given in (5.2)-(5.8), together with Lemmas 5.3.5-5.3.9, enable us to make reasonably straightforward, though tedious, calculations of the orders in (5.14). An important point to note is that the comparison between $B_{1} \cap X Y$ and $\left(B_{1} \cap X\right)\left(B_{1} \cap Y\right)$, and between $B_{1} \cap U V$ and $\left(B_{1} \cap U\right)\left(B_{1} \cap V\right)$, is determined in the maximal Cartesian submodule of $B_{1}$.

Write $B_{1}=F(i, j, k, l, \delta, \xi, \alpha)$ as in Theorem 5.2.4. If $\delta_{1}=1$ or $\delta_{2}=0$ then $d_{2}^{0,1}=0$ by Lemma 5.3.5. If $\delta=(0,1)$ then $d_{2}^{0,1} \neq 0$ if and only if $\xi=1$ or -1 , in which case coker $d_{2}^{0,1}=0$ by Lemma 5.3.5. When $d_{2}^{0,1}=0, \operatorname{coker} d_{2}^{0,1}=H^{2}\left(\langle b\rangle, B_{1}^{N}\right)$, which is described by (5.13).

By Lemma 5.3.6, $d_{2}^{1,1}=0$ unless $\delta=(0,1)$ and either $\xi=0$ or $\xi=-1$. If $d_{2}^{1,1}$ is nonzero then $\left|\operatorname{ker} d_{2}^{1,1}\right|=\left|E_{2}^{1,1}\right| / 2$, also by Lemma 5.3.6. Now even as $\langle b\rangle$-modules, $H^{1}\left(\langle a\rangle, B_{1}\right)$ is isomorphic to the section $B_{1} \cap U V / B_{1}^{1-a}$ of $B_{1}$. Also note that $H^{1}\left(\langle a\rangle, B_{1}\right)$ is elementary abelian. (This is a consequence of the following fact: for a finite group $G$, a $G$-module $M$ and all $i \geq 1, H^{i}(G, M)$ has exponent at most $|G|$. A proof of this fact may be found in Robinson (1982), p.326, 11.3.7.) Since $\operatorname{rk}\left(B_{1} \cap U V\right)=2, H^{1}\left(\langle a\rangle, B_{1}\right)$ has order at most 4. If $B_{1} \cap U V=\left(B_{1} \cap U\right)\left(B_{1} \cap V\right)$ then every element of $H^{1}\left(\langle a\rangle, B_{1}\right)$ is fixed (hence inverted) by $b$, so that $E_{2}^{1,1}=H^{1}\left(\langle a\rangle, B_{1}\right)$. Suppose that $B_{1} \cap U V \neq\left(B_{1} \cap U\right)\left(B_{1} \cap V\right)$. If $H^{1}\left(\langle a\rangle, B_{1}\right) \cong C_{2}$ then clearly $E_{2}^{1,1}=H^{1}\left(\langle a\rangle, B_{1}\right)$. Otherwise, we claim that $E_{2}^{1,1}=0$. Of course, this needs to be verified only when $H^{1}\left(\langle a\rangle, B_{1}\right) \cong C_{2} \times C_{2}$. In that case, $b$ cannot fix every element of $H^{1}\left(\langle a\rangle, B_{1}\right)$ : if it did, we would have $B_{1}^{1-a}=\left(B_{1} \cap U\right)\left(B_{1} \cap V\right)$ and hence $B_{1} \cap U V=\left(B_{1} \cap U\right)\left(B_{1} \cap V\right)$, contrary to assumption. Thus, on $H^{1}\left(\langle a\rangle, B_{1}\right), 1+b$ has kernel $C_{2}$ and $1-b$ has image $C_{2}$, that is, $E_{2}^{1,1}=0$. Now note that by Remark 3.1.9, $H^{i}\left(\langle a\rangle, B_{1}\right) \cong H^{i+1}\left(\langle a\rangle, B_{1}\right)[$ as groups, not necessarily as modules]. The discussion of this paragraph is summarised as follows:

$$
E_{2}^{1,1}=\left\{\begin{array}{cl}
H^{2}\left(\langle a\rangle, B_{1}\right) & \text { if } B_{1} \cap U V=\left(B_{1} \cap U\right)\left(B_{1} \cap V\right) \\
& \text { or } H^{2}\left(\langle a\rangle, B_{1}\right) \cong C_{2} \\
0 & \text { otherwise. }
\end{array}\right.
$$


By Corollary 5.3.8, $d_{2}^{0,2}=0$ unless $\delta=(1,0)$ and $\xi=-1$. If $d_{2}^{0,1} \neq 0$ then $\left|\operatorname{ker} d_{2}^{0,2}\right|=\left|E_{2}^{0,2}\right| / 2$. By reasoning similar to that used in the previous paragraph,

$$
E_{2}^{0,2}=\left\{\begin{array}{cl}
H^{2}\left(\langle a\rangle, B_{1}\right) & \text { if } B_{1} \cap X Y=\left(B_{1} \cap X\right)\left(B_{1} \cap Y\right) \\
& \text { or } H^{2}\left(\langle a\rangle, B_{1}\right)=0 \\
C_{2} & \text { otherwise. }
\end{array}\right.
$$

All that remains is to calculate $H^{2}\left(\langle a\rangle, B_{1}\right)$, which is elementary abelian of order at most 4. If $B_{1}$ is Cartesian then $B_{1}$ is the sum of rank 2 submodules $B_{1} \cap X Y$ and $B_{1} \cap U V$, which are respectively fixed and inverted by $a$; thus, $\left|H^{2}\left(\langle a\rangle, B_{1}\right)\right|=4$. If the unique maximal Cartesian submodule of $B_{1}$ has index 2, then $B_{1}$ arises from an isomorphism between length 1 sections of $X Y / F_{a}$ and $U V / F_{a}$. Thus $B_{1}^{1+a}$ has index 2 in $B_{1} \cap X Y$ and $\left|H^{2}\left(\langle a\rangle, B_{1}\right)\right|=2$. Finally, if the unique maximal Cartesian has index 4 in $B_{1}$, then $\left|H^{2}\left(\langle a\rangle, B_{1}\right)\right|=1$. From the discussion following Theorem 5.2.4, it is easy to recognise the index of the maximal Cartesian submodule in $B_{1}$, given the label of $B_{1}$ in our scheme.

\begin{tabular}{|c|c|c|c|c|}
\hline$B_{1}$ & $\left|\operatorname{coker} d_{2}^{0,1}\right|$ & $\left|\operatorname{ker} d_{2}^{1,1}\right|$ & $\left|\operatorname{ker} d_{2}^{0,2}\right|$ & $\left|H^{2}\left(V_{4}, B_{1}\right)\right|$ \\
\hline$F(i, j, k, l, 0,0)$ & \multirow{4}{*}{2} & 4 & 4 & 32 \\
\hline$F(i, j, k, l, 0,0, \xi)$ & & 2 & 2 & 8 \\
\hline$F(i, j, k, l, 0,0,2, \alpha)$ & & \multirow{2}{*}{1} & 1 & 2 \\
\hline$F(i, j, k, l, 0,1)$ & & & 4 & 8 \\
\hline$F(i, j, k, l, 0,1,1)$ & \multirow{2}{*}{1} & 2 & \multirow{5}{*}{2} & 4 \\
\hline$F(i, j, k, l, 0,1,-1)$ & & \multirow{2}{*}{1} & & 2 \\
\hline$F(i, j, k, l, 0,1,0)$ & 2 & & & 4 \\
\hline$F(i, j, k, l, 1,0)$ & \multirow{7}{*}{1} & 4 & & 8 \\
\hline$F(i, j, k, l, 1,0,1)$ & & \multirow{3}{*}{2} & & 4 \\
\hline$F(i, j, k, l, 1,0,-1)$ & & & 1 & 2 \\
\hline$F(i, j, k, l, 1,0,0)$ & & & \multirow{3}{*}{2} & 4 \\
\hline$F(i, j, k, l, 1,1)$ & & 1 & & 2 \\
\hline$F(i, j, k, l, 1,1,1)$ & & 2 & & 4 \\
\hline$F(i, j, k, l, 1,1,2, \alpha)$ & & 1 & 1 & 1 \\
\hline
\end{tabular}

Table 5.1: Calculation of $\left|H^{2}\left(V_{\mathbf{4}}, B_{1}\right)\right|$ 
We use the discussion above to calculate the orders in (5.14) - see Table 5.1. The order of $H^{2}\left(V_{4}, B_{1}\right)$ is obtained by reading across the row in which $B_{1}$ is listed, and multiplying the values in the second, third and fourth columns.

The next lemma is useful here and in the extension problem for $T=C$. Conjugacy in $G L(4)$ will be denoted by $\sim$. When we need to specify conjugacy in a subgroup $S$ of $G L(4)$, we write $\sim_{S}$.

Lemma 5.3.10 Let $G$ be a finite 2 -subgroup of $B S_{4}$ such that $B G=B T$ for some regular subgroup $T$ of $S_{4}$. If $G$ splits over $B \cap G$ then $G \sim_{B T}(B \cap G) \rtimes T$.

Proof. If $K$ is a complement of $B \cap G$ in $G$ then $K$ is a complement of $B$ in $B T \cong C_{2 \infty}$ wr $T$. Any two complements of the base group $B$ in this wreath product are conjugate (see Neumann (1964), Theorem 10.1). Since $T$ itself is such a complement, the result follows.

Theorem 5.3.11 A finite subgroup $G$ of $B V_{4}$ such that $B G=B V_{4}$ is $B S_{4}$ conjugate to one of the groups in the following list:

$$
\begin{aligned}
& \left\langle a x_{i+1}^{\varepsilon} y_{j+1}^{\eta}, b x_{i+1}^{\gamma} u_{k+1}^{\mu} v_{l+1}^{\nu}, F(i, j, k, l, 0,0)\right\rangle ; \\
& \left\langle a y_{j+1}^{\eta}, b x_{i+1}^{\gamma} u_{k+1}^{\mu}, F(i, j, k, l, 0,0,1-1)\right\rangle ; \\
& \left\langle a x_{i+1}^{\varepsilon}, b x_{i+1}^{\gamma} u_{k+1}^{\mu}, F(i, j, k, l, 0,0, \xi,-1)\right\rangle, \xi \in\{0,-1\} ; \\
& \left\langle a x_{i+1}^{\varepsilon} y_{j+1}^{\eta}, b x_{i+1}^{\gamma}, F(i, j, k, l, 0,1)\right\rangle ; \\
& \left\langle a y_{j+1}^{\eta}, b\left(x_{i+2} u_{2} v_{2}\right)^{\gamma}, F(i, j, 0,0,0,1,1)\right\rangle ; \\
& \left\langle a x_{i+1}^{\varepsilon}, b, F(i, j, k, l, 0,1,-1)\right\rangle ; \\
& \left\langle a x_{i+1}^{\varepsilon}, b x_{i+1}^{\gamma}, F(i, j, k, l, 0,1,0)\right\rangle ; \\
& \left\langle a x_{i+1}^{\varepsilon}, b u_{k+1}^{\mu} v_{l+1}^{\nu}, F(i, j, k, l, 1,0)\right\rangle ; \\
& \left\langle a\left(x_{i+2} y_{j+2}\right)^{\varepsilon}, b\left(x_{i+2} u_{k+2}\right)^{\varepsilon} v_{l+1}^{\nu}, F(i, j, k, l, 1,0,1)\right\rangle ; \\
& \left\langle a, b v_{l+1}^{\nu}, F(i, j, k, l, 1,0,-1)\right\rangle ; \\
& \left\langle a x_{i+1}^{\varepsilon}, b, F(i, j, k, l, 1,1)\right\rangle ; \\
& \left\langle a\left(x_{i+2} y_{j+2}\right)^{\varepsilon}, b\left(x_{i+2} u_{k+2}\right)^{\gamma} v_{l+2}^{\varepsilon-\gamma}, F(i, j, k, l, 1,1,1)\right\rangle ; \\
& \langle a, b, F(i, j, k, l, 1,1,2, \alpha)\rangle, \alpha \in\{1,-1\} ;
\end{aligned}
$$

where the parameters $\varepsilon, \eta, \gamma, \mu, \nu$ range over $\{0,1\}$ and the $i, j, k, l$ range over the non-negative integers under the conditions given before Theorem 5.2.4. No two groups in this list are $B V_{4}$-conjugate. 
Proof. For the purposes of the theorem, we may assume that $B \cap G=B_{1}$ is in the list of Proposition 5.2.8. We claim that for some $x_{a}, x_{b} \in X, y \in Y, u \in U$ and $v \in V$

$$
G \sim_{B}\left\langle a x_{a} y, b x_{b} u v, B_{1}\right\rangle .
$$

To see this, first note that, since $G$ has projection group $V_{4}$ and $B=X Y U V$,

$$
G=\left\langle a x_{a} y \tilde{u} \tilde{v}, b x_{b} \tilde{y} u v, B_{1}\right\rangle
$$

for some $\tilde{y} \in Y, \tilde{u} \in U$ and $\tilde{v} \in V$. Then choose $y^{\prime} \in Y, u^{\prime} \in U, v^{\prime} \in V$ such that $y^{\prime 2}=\tilde{y}^{-1}, u^{\prime 2}=\tilde{u}^{-1}, v^{\prime 2}=\tilde{v}^{-1}$ and note that

$$
G^{y^{\prime} u^{\prime} v^{\prime}}=\left\langle a x_{a} y, b x_{b} u v \tilde{v}^{-1}, B_{1}\right\rangle
$$

this verifies the claim.

The relations $a^{2}=b^{2}=1$ in $V_{4}$ imply that

$$
\begin{aligned}
& x_{a}^{2} y^{2} \in B_{1} \cap X Y, \\
& x_{b}^{2} u^{2} \in B_{1} \cap X U .
\end{aligned}
$$

The relation $[a, b]=1$ in $V_{4}$ implies that $y^{-2} u^{2} v^{2} \in B_{1} \cap Y U V$. However, $y^{4} \in B_{1} \cap Y$ by $(5.15)$, since $\left(B_{1} \cap X\right)\left(B_{1} \cap Y\right)$ has index at most 2 in $B_{1} \cap X Y$. Therefore, we write the last condition more conveniently as

$$
y^{2} u^{2} v^{2} \in B_{1} \cap Y U V
$$

Conversely, any group generated by $B_{1}, a x_{a} y, b x_{b} u v$, satisfying (5.15)-(5.17), is a finite supplement of $B$ in $B V_{4}$. The proof proceeds by showing, for each choice of $B_{1}$, that $G$ is $B V_{4}$-conjugate to at least one of, and hence (by Theorem 5.3.3) precisely one of $\left|H^{2}\left(V_{4}, B_{1}\right)\right|$ groups. Because of its repetitive nature, we illustrate this proof only in a few (typical) cases. The notation $\equiv$ will denote equality modulo $B_{1}$, unless stated otherwise.

Write $B_{1}=F(i, j, k, l, \delta, \xi, \alpha)$ and, to begin with, suppose that $\delta=(0,0)$. Then $B_{1} \cap X Y=\left(B_{1} \cap X\right)\left(B_{1} \cap Y\right)$ and by (5.15), $x_{a}^{2} \in B_{1} \cap X=\left\langle x_{i}\right\rangle$ and $y^{2} \in B_{1} \cap Y=\left\langle y_{j}\right\rangle$. That is, $x_{a} \equiv 1$ or $x_{i+1}$ and $y \equiv 1$ or $y_{j+1}$. In fact, we may take $x_{a}=1$ or $x_{i+1}$ and $y=1$ or $y_{j+1}$. Similarly, since $B_{1} \cap U V=\left(B_{1} \cap U\right)\left(B_{1} \cap V\right)$ in this case, by (5.17) we may take $u=1$ or $u_{k+1}$ and $v=1$ or $v_{l+1}$. By (5.16), $x_{b}=1$ or $x_{i+1}$. These choices give us 32 extensions of $B_{1}$ by $V_{4}$ in $B V_{4}$. If $B_{1}$ 
is Cartesian then, by Theorem 5.3.3 and Table 5.1, no two of these extensions are $B V_{4}$-conjugate. If $B_{1}$ is diagonal and $\xi=(1,-1)$, then $x_{i+1} \equiv u_{k+1} v_{l+1}$ and

$$
\begin{array}{rll}
\left\langle a x_{i+1} y, b x_{b} u v, B_{1}\right\rangle & = & \left\langle a y u_{k+1} v_{l+1}, b x_{b} u v, B_{1}\right\rangle \\
& \sim_{u_{k+2} v_{l+2}} & \left\langle a y, b x_{b} u v v_{l+1}, B_{1}\right\rangle .
\end{array}
$$

Furthermore,

$$
\left\langle a y, b x_{b} u v_{l+1}, B_{1}\right\rangle=\left\langle a y, b x_{b} x_{i+1} u u_{k+1}, B_{1}\right\rangle .
$$

By Theorem 5.3.3 and Table 5.1, this shows that $G$ is $B V_{4}$-conjugate to one and only one of the eight groups obtained by letting $y, x_{b}, u$ in $\left\langle a y, b x_{b} u, B_{1}\right\rangle$ range over the values indicated above.

Now suppose that $\delta=(0,1)$. Then we have the same possibilities for $x_{a}$ and $y$ as in the case $\delta=(0,0)$. If $\xi=-1$ then $B_{1} \cap X U=\left(B_{1} \cap X\right)\left(B_{1} \cap U\right)$. By (5.16) and (5.17), we also have the same possibilities for $x_{b}, u$ and $v$ as in the case $\delta=(0,0)$. However, here we have $y_{j+1} \equiv x_{i+1} u_{k+1}$, so that

$$
\left\langle a x_{a} y_{j+1}, b x_{b} u v, B_{1}\right\rangle \sim_{u_{k+2}}\left\langle a x_{a}, b v, B_{1}\right\rangle
$$

and

$$
\left\langle a x_{a}, b x_{i+1} u v, B_{1}\right\rangle \sim_{y_{j+2}}\left\langle a x_{a}, b u u_{k+1} v, B_{1}\right\rangle
$$

Since $u_{k+1} \equiv v_{l+1}$ also in this case,

$$
G \sim_{B}\left\langle a x_{a}, b v, B_{1}\right\rangle
$$

In fact, since $\left\langle a x_{a}, b v_{l+1}, B_{1}\right\rangle \sim_{u_{k+2} v_{l+2}}\left\langle a x_{a} u_{k+1} v_{l+1}, b, B_{1}\right\rangle=\left\langle a x_{a}, b, B_{1}\right\rangle$, we have

$$
G \sim_{B}\left\langle a x_{a}, b, B_{1}\right\rangle
$$

By Theorem 5.3.3 and Table 5.1, these latter two groups are not $B V_{4}$-conjugate.

Now suppose that $\delta=(1,0)$ and $\xi=1$. In this case $B_{1} \cap X Y \neq\left(B_{1} \cap X\right)\left(B_{1} \cap Y\right)$ and $B_{1} \cap X U \neq\left(B_{1} \cap X\right)\left(B_{1} \cap U\right)$. By (5.15), $x_{a}^{4} \in B_{1} \cap X$ and $y^{4} \in B_{1} \cap Y$, so that $x_{a} y \equiv 1$ or $x_{i+2} y_{j+2} \bmod \left\langle x_{i+1}, y_{j+1}\right\rangle$. Similarly, by $(5.16), x_{b} u \equiv 1$ or $x_{i+2} u_{k+2}$ $\bmod \left\langle x_{i+1}, u_{k+1}\right\rangle$. Since $x_{i+1} \equiv y_{j+1} \equiv u_{k+1}$, after conjugation by $u_{k+2} y_{j+2}$ we may take $x_{a} y=1$ or $x_{i+2} y_{j+2}$ and $x_{b} u=1$ or $x_{i+2} u_{k+2}$. When $x_{b} u=x_{i+2} u_{k+2}$, we have $x_{a} y=x_{i+2} y_{j+2}$ and $v=1$ or $v_{l+1}$ by (5.17). When $x_{b} u=1$, we have $x_{a} y=1$ and $v=1$ or $v_{l+2}$. The four extensions of $B_{1}$ so obtained are pairwise non-conjugate in $B V_{4}$ by Theorem 5.3.3 and Table 5.1. 
We remark that no calculation is necessary when $B_{1}=F(i, j, k, l, 1,1,2, \alpha)$. For by Table $5.1, G$ splits over $B_{1}$ in this case and we may appeal to Lemma 5.3.10.

We have proved that no two distinct groups in the list of the theorem with the same diagonal subgroup are $B V_{4}$-conjugate. Therefore, no two distinct groups in the list can be $B V_{4}$-conjugate, since this would imply that they had the same diagonal subgroup.

\subsection{The conjugacy problem}

Consider two finite irreducible 2-subgroups of $B S_{4}$ which are $G L(4)$-conjugate. If the conjugating matrix maps diagonal subgroup onto diagonal subgroup then, by Theorem 2.4.1, these diagonal subgroups are $S_{4}$-conjugate. If diagonal subgroup is not mapped onto diagonal subgroup, then each of these irreducible groups has a selfcentralising normal subgroup with quotient isomorphic to the projection group, different from the diagonal subgroup. We exploit this fact in the following procedure for choosing a complete and irredundant set of representatives of the $G L(4)$-conjugacy classes of the finite irreducible subgroups of $B V_{4}$.

Step 1. From the set of $B V_{4}$-conjugacy class representatives given in Theorem 5.3.11, we choose representatives of the $B S_{4}$-conjugacy classes. This is done in Theorem 5.4.1. The choice is made in such a way that two representatives with different diagonal subgroups cannot have $S_{4}$-conjugate diagonal subgroups.

Step 2. We determine which of the groups listed in Theorem 5.4.1 have only one self-centralising normal subgroup with quotient $V_{4}$ (in the sequel, such subgroups will be referred to by the acronym SCN4). We claim that two distinct groups of this kind in that list are not $G L(4)$-conjugate. For if we had $G^{m}=H$ for two such groups $G$ and $H$ and $m \in G L(4)$, then by the discussion before step 1 and the property of the list mentioned in step 1 , we would have $B \cap G=B \cap H$ and moreover $(B \cap G)^{m}=B \cap G$. By Proposition 2.3.7 and Remark 2.3.8, this would imply that $m \in B S_{4}$. But by choice, $G$ and $H$ lie in different $B S_{4}$-conjugacy classes. This verifies the claim. Also note that, of course, none of these groups is isomorphic to any group which has more than one SCN4 subgroup.

Step 3. From among those groups in the list of Theorem 5.4.1 which have more than one SCN4 subgroup, we select representatives of $G L(4)$-conjugacy classes. Together with all the groups in the list which have only one SCN4 subgroup, these 
will then form the desired set.

We use the Kronecker delta notation in the following result. There should be no confusion with the parameter $\delta$ in the label of a finite $V_{4}$-submodule of $B$.

Theorem 5.4.1 For $\varepsilon, \eta, \gamma, \mu, \nu$ ranging over $\{0,1\}$, the following is a complete and irredundant list of $B S_{4}$-conjugacy class representatives of the finite irreducible subgroups of $B V_{4}$.

For $i, j, k, l \geq 1$ and $\xi \in\{-1,0\}$ :

$$
\begin{array}{lll}
\left\langle a x_{i+1}^{\varepsilon} y_{j+1}^{\eta}, b x_{i+1}^{\gamma} u_{k+1}^{\mu} v_{l+1}^{\nu}, F(i, j, k, l, 0,0)\right\rangle, & j<k<l ; \\
\left\langle a x_{i+1}^{\varepsilon} y_{j+1}^{\eta}, b x_{i+1}^{\gamma(1-\varepsilon)} u_{k+1}^{\mu} v_{k+1}^{\nu \cdot \mu^{1-\epsilon}}, F(i, j, k, k, 0,0)\right\rangle, & j \neq k ; \\
\left\langle a x_{i+1}^{\varepsilon} y_{j+1}^{\eta}, b u_{j+1}^{\mu^{1-\eta(1-\epsilon)}} v_{j+1}^{\nu \cdot \eta^{1-\varepsilon}}, F(i, j, j, j, 0,0)\right\rangle ; & \\
\left\langle a y_{j+1}^{\eta}, b x_{i+1}^{\gamma\left(1-\mu . \delta_{k, l}\right)} u_{k+1}^{\mu}, F(i, j, k, l, 0,0,1,-1)\right\rangle, & k \leq l ; \\
\left\langle a x_{i+1}^{\varepsilon}, b x_{i+1}^{\gamma} u_{k+1}^{\mu}, F(i, j, k, l, 0,0, \xi,-1)\right\rangle, & j<k<l ; \\
\left\langle a x_{i+1}^{\varepsilon}, b x_{i+1}^{\gamma(1-\varepsilon)\left(1-\delta_{j, k}\right)} u_{k+1}^{\mu}, F(i, j, k, k, 0,0, \xi,-1)\right\rangle ; & \\
\left\langle a x_{i+1}^{\varepsilon} y_{j+1}^{\eta}, b x_{i+1}^{\gamma\left(1-\varepsilon . \delta_{k, l}\right)}, F(i, j, k, l, 0,1)\right\rangle, & k \leq l ; \\
\left\langle a y_{j+1}^{\eta}, b\left(x_{i+2} u_{2} v_{2}\right)^{\gamma}, F(i, j, 0,0,0,1,1)\right\rangle ; &
\end{array}
$$

for $i, j \geq 1$ and $k, l \gtrsim 0$ :

$$
\begin{array}{ll}
\left\langle a x_{i+1}^{\varepsilon}, b, F(i, j, k, l, 0,1,-1)\right\rangle, & k \leq l ; \\
\left\langle a x_{i+1}^{\varepsilon}, b x_{i+1}^{\gamma}, F(i, j, k, l, 0,1,0)\right\rangle, & j<k<l \text { if } k \neq 0 ; \\
\left\langle a x_{i+1}^{\varepsilon}, b x_{i+1}^{\gamma(1-\varepsilon)\left(1-\delta_{j, k}\right)}, F(i, j, k, k, 0,1,0)\right\rangle ; &
\end{array}
$$

for $i, j \gtrsim 0$ and $k, l \geq 1$ :

$$
\begin{array}{ll}
\left\langle a x_{i+1}^{\varepsilon}, b u_{k+1}^{\mu} v_{l+1}^{\nu \cdot \phi^{\delta} k, l}, F(i, j, k, l, 1,0)\right\rangle, & k \leq l ; \\
\left\langle a\left(x_{i+2} y_{j+2}\right)^{\varepsilon}, b\left(x_{i+2} u_{k+2}\right)^{\varepsilon} v_{l+1}^{\nu}, F(i, j, k, l, 1,0,1)\right\rangle, & j \leq k ; \\
\left\langle a, b v_{l+1}^{\nu}, F(i, j, k, l, 1,0,-1)\right\rangle, & k \leq l ; \\
\left\langle a x_{i+1}^{\varepsilon}, b, F(i, j, k, l, 1,1)\right\rangle, & k \leq l ;
\end{array}
$$

for $i, j \gtrsim 0, k, l \gtrsim 0$ and $\alpha \in\{-1,1\}$ :

$$
\begin{array}{ll}
\left\langle a\left(x_{i+2} y_{j+2}\right)^{\varepsilon}, b\left(x_{i+2} u_{k+2}\right)^{\gamma} v_{l+2}^{\varepsilon-\gamma}, F(i, j, k, l, 1,1,1)\right\rangle, & j<k<l ; \\
\left\langle a\left(x_{i+2} y_{j+2}\right)^{\varepsilon}, b\left(x_{i+2} u_{k+2}\right)^{\gamma(1-\varepsilon)} v_{l+2}^{\gamma-\varepsilon}, F(i, j, k, k, 1,1,1)\right\rangle, & j \neq k ; \\
\left\langle a, b\left(x_{i+2} u_{j+2} v_{j+2}\right)^{\gamma}, F(i, j, j, j, 1,1,1)\right\rangle ; & \\
\langle a, b, F(i, j, k, l, 1,1,2, \alpha)\rangle, & k \leq l, \text { and } j \leq k \text { if } i \neq 0 .
\end{array}
$$

Proof. Observe first that we have here a sublist of the list given in Theorem 5.3.11, and also (by Lemma 5.2.6) this sublist consists of irreducible groups. The rest of 
the proof amounts to comparison with that list, once we know how to recognize the intersections of the $B S_{4}$-conjugacy classes with the set of groups in that list. Note that for each $G$ in the list of Theorem 5.3.11, $B \cap G$ is immediately visible and is in the modified list of Proposition 5.2.8. Let $G$ be any irreducible group listed in Theorem 5.3.11. Since $B S_{4}=B V_{4} S_{3}=S_{3}\left(B S_{4}\right)$ (where, as before, $S_{3}$ stands for the stabilizer of 4 in $S_{4}$ ), we need to recognize those $G^{s t}$ with $s \in S_{3}$ and $t \in B V_{4}$ which are also in the list of Theorem 5.3.11. Since

$$
B \cap G^{s} \unlhd B G^{s}=(B G)^{s}=\left(B V_{4}\right)^{s}=B V_{4},
$$

we have

$$
B \cap G^{s t}=(B \cap G)^{s t}=(B \cap G)^{s}=B \cap G^{s} .
$$

Since $G$ and $G^{s t}$ are assumed listed in Theorem 5.3.11, $B \cap G$ and $B \cap G^{\boldsymbol{s}}$ must be in the modified list of Proposition 5.2.8. From the orbit list given in the proof of Proposition 5.2 .8 (which is really an $S_{3}$-orbit list) we see that, for $B \cap G$ in the first seven lines, this restricts $s$ to two choices, but otherwise all six choices are allowed. For each nontrivial choice of $s$ so allowed, we have to calculate $G^{s}$ and then find the unique $B V_{4}$-conjugate (in fact $B$-conjugate) of $G^{s}$ in the list of Theorem 5.3.11. This will identify all the $B S_{4}$-conjugates of $G$ in that list, and then inspection shows that precisely one of these groups is listed in Theorem 5.3.11.

We illustrate this process for two particular choices of $G$, and omit the similar calculations in the remaining cases. First, let

$$
G=\left\langle a x_{i+1}^{\varepsilon} y_{j+1}^{\eta}, b x_{i+1}^{\gamma}, F(i, j, k, l, 0,1)\right\rangle
$$

From the orbit list in the proof of Proposition 5.2.8, we see that the only nontrivial element of $S_{3}$ which is relevant here is (12), and so it suffices to consider

$$
\begin{aligned}
G^{(12)} & =\left\langle a x_{i+1}^{\varepsilon} y_{j+1}^{\eta}, a b x_{i+1}^{\gamma}, F(i, j, l, k, 0,1)\right\rangle \\
& =\left\langle a x_{i+1}^{\varepsilon} y_{j+1}^{\eta}, b x_{i+1}^{\gamma+\varepsilon} y_{j+1}^{\eta}, F(i, j, l, k, 0,1)\right\rangle .
\end{aligned}
$$

When $\gamma+\varepsilon$ is 2 it may be replaced by 0 , since $x_{i+1}^{2} \in F(i, j, k, l, 0,0)$. (Similar steps may be used in other cases as well: $\gamma+\varepsilon$ is always read modulo 2 , so that this expression stays within the range $\{0,1\}$.) The unique $B V_{4}$-conjugate of $G^{(12)}$ must be in the same line of the list of Theorem 5.3.11 as $G$, so in this case we seek a $B V_{4}$-conjugate whose second listed generator involves no factor from $Y$. Clearly,

$$
G^{(12)} \sim_{y_{j+2}^{-\eta}}\left\langle a x_{i+1}^{\varepsilon} y_{j+1}^{\eta}, b x_{i+1}^{\gamma+\varepsilon}, F(i, j, l, k, 0,1)\right\rangle
$$


which (with the informal convention that $\gamma+\varepsilon$ is read modulo 2 ) is in the list of Theorem 5.3.11, as required. This group and $G$ form the intersection of that list with one $B S_{4}$-conjugacy class, so we have to check that one and only one of them appears in the list of Theorem 5.4.1. Indeed, if $k<l$ then $G$ is there and the other group is not, whereas if $l<k$ then $G$ is not there but the other group is. When $k=l$ and $\varepsilon=1$, the two groups differ only in their second listed generator; in one or the other group (depending on the value of $\gamma$ ) it is simply $b$; that group is there, the other is not. When $k=l$ and $\varepsilon=0$, the two groups are the same and this one group is there.

The second case we consider is

$$
G=\left\langle a x_{i+1}^{\varepsilon}, b x_{i+1}^{\gamma} u_{k+1}^{\mu}, F(i, j, k, l, 0,0,0,-1)\right\rangle .
$$

By inspection of the orbit list in the proof of Proposition 5.2.8, we see that all six elements of $S_{3}$ are relevant here. When $j, k, l$ are pairwise different, the $\operatorname{six} S_{3}$ conjugates of $G$ differ even in their intersections with $B$, but at that level only by permutations of the three relevant parameters, and precisely one of them has those parameters in increasing order. Passing to the unique $B V_{4}$-conjugates in the list of Theorem 5.3.11 has no effect on those three parameters. Hence, there we find six distinct groups, all in the third line (and with $\xi=0$ ), precisely one with the three parameters in increasing order. It is not necessary to calculate how the values of the parameters $\varepsilon, \gamma, \mu$ of the six groups depend on the parameter values of $G$, since it follows from what has been said so far that precisely one of them is in the list of Theorem 5.4.1.

The determination is not so easy when two of $j, k, l$ are equal but differ from the third: for example, when $j \neq k=l$. In this case, $G$ has either three or six distinct conjugates in the list of Theorem 5.3.11, and among these only one or two, namely $G$ and $G^{(12)}$ (which may or may not be distinct), have the three critical parameters in the order allowed in Theorem 5.4.1. We have

$$
\begin{aligned}
G^{(12)} & =\left\langle a x_{i+1}^{\varepsilon}, a b x_{i+1}^{\gamma} v_{k+1}^{\mu}, F(i, j, k, k, 0,0,0,-1)\right\rangle \\
& =\left\langle a x_{i+1}^{\varepsilon}, b x_{i+1}^{\gamma+\varepsilon} v_{k+1}^{\mu}, F(i, j, k, k, 0,0,0,-1)\right\rangle \\
& =\left\langle a x_{i+1}^{\varepsilon}, b x_{i+1}^{\gamma+\varepsilon} y_{j+1}^{\mu} u_{k+1}^{\mu}, F(i, j, k, k, 0,0,0,-1)\right\rangle,
\end{aligned}
$$

using the fact that $y_{j+1} u_{k+1} v_{k+1}^{-1} \in F(i, j, k, k, 0,0,0,-1)$. Then we see that the $B V_{4}$ conjugate of $G^{(12)}$ in the list of Theorem 5.3 .11 is the conjugate by $y_{j+2}^{-\mu}$, namely

$$
\left\langle a x_{i+1}^{\varepsilon}, b x_{i+1}^{\gamma+\varepsilon} u_{k+1}^{\mu}, F(i, j, k, k, 0,0,0,-1)\right\rangle
$$


(again with the convention that $\gamma+\varepsilon$ is read modulo 2 ). We have to verify that precisely one of $G$ and this group is listed in Theorem 5.4.1. When $\varepsilon=1$, the two groups differ only in their second listed generator: in one or the other group (depending on the value of $\gamma$ ) it is simply $b u_{k+1}^{\mu}$; that group is there, the other is not. When $\varepsilon=0$, the two groups are the same and this one group is there.

When $j=k=l$, we need to calculate explicitly all six (not necessarily distinct) $B S_{4}$-conjugates of $G$ in the list of Theorem 5.3.11. Both $G$ and $G^{(12)}$ are already known; these are now

$$
\begin{aligned}
& \left\langle a x_{i+1}^{\varepsilon}, b x_{i+1}^{\gamma} u_{j+1}^{\mu}, F(i, j, j, j, 0,0,0,-1)\right\rangle, \\
& \left\langle a x_{i+1}^{\varepsilon}, b x_{i+1}^{\gamma+\varepsilon} u_{j+1}^{\mu}, F(i, j, j, j, 0,0,0,-1)\right\rangle,
\end{aligned}
$$

respectively. Next,

$$
G^{(23)}=\left\langle a x_{i+1}^{\gamma} y_{j+1}^{\mu}, b x_{i+1}^{\varepsilon}, F(i, j, j, j, 0,0,0,-1)\right\rangle
$$

and so

$$
G^{(23)} \sim_{y_{j+2}^{\mu} u_{j+2}^{\mu} v_{j+2}^{\mu}}\left\langle a x_{i+1}^{\gamma} y_{j+1}^{\mu} u_{j+1}^{\mu} v_{j+1}^{\mu}, b x_{i+1}^{\varepsilon} y_{j+1}^{\mu} v_{j+1}^{\mu}, F(i, j, j, j, 0,0,0,-1)\right\rangle
$$

Since $y_{j+1} u_{j+1} v_{j+1} \in F(i, j, j, j, 0,0,0,-1)$, this last group may be written as

$$
\left\langle a x_{i+1}^{\gamma}, b x_{i+1}^{\varepsilon} u_{j+1}^{\mu}, F(i, j, j, j, 0,0,0,-1)\right\rangle .
$$

Applying to (5.20) the process which led from (5.18) to (5.19), then to (5.19) the process which led from $(5.18)$ to $(5.20)$, and so on, we obtain the other three $B S_{4}$ conjugates of $G$ in the list of Theorem 5.3.11:

$$
\begin{aligned}
& \left\langle a x_{i+1}^{\gamma}, b x_{i+1}^{\varepsilon+\gamma} u_{j+1}^{\mu}, F(i, j, j, j, 0,0,0,-1)\right\rangle, \\
& \left\langle a x_{i+1}^{\gamma+\varepsilon}, b x_{i+1}^{\varepsilon} u_{j+1}^{\mu}, F(i, j, j, j, 0,0,0,-1)\right\rangle, \\
& \left\langle a x_{i+1}^{\varepsilon+\gamma}, b x_{i+1}^{\gamma} u_{j+1}^{\mu}, F(i, j, j, j, 0,0,0,-1)\right\rangle .
\end{aligned}
$$

(As before, $\varepsilon+\gamma$ is to be read modulo 2.) When $\varepsilon=\gamma=0$, these six groups are the same, and that group is in the list. For fixed $\mu, i, j$, the groups $G$ obtained with the other three choices for the pair $(\varepsilon, \gamma)$ fall into a single $B S_{4}$-conjugacy class, and of these three precisely one (namely that corresponding to $\varepsilon=1, \gamma=0$ ) appears in the list of Theorem 5.4.1. 
Remark 5.4.2 By comparing the list of Theorem 5.4.1 and the orbit list in the proof of Proposition 5.2.8, it may be seen that if two groups in Theorem 5.4.1 have distinct diagonal subgroups, then these diagonal subgroups are not $S_{4}$-conjugate. (Essentially, we have considered only extensions of $S_{4}$-conjugacy class representatives of finite $V_{4}$-submodules of $B$.)

Remark 5.4.3 By Remark 2.3.8, no two groups in the list of Theorem 5.4.1 are even $M(4)$-conjugate.

This completes step 1 . Now we begin to consider step 2 .

Lemma 5.4.4 Let $G$ and $H$ be groups in the list of Theorem 5.4.1 and suppose that $\theta: G \rightarrow H$ is an isomorphism such that $(B \cap G) \theta \neq B \cap H$.

(i) If $(B \cap G) \cdot(B \cap H) \theta^{-1} \neq G$, set $Q=(B \cap G) \cap(B \cap H) \theta^{-1}$ : then $Q$ is a $V_{4}$-submodule of index 2 in $B \cap G$ such that $\left|C_{V_{4}}(Q)\right|=2$ and $B .(B \cap H) \theta^{-1}=B C_{V_{\mathbf{4}}}(Q)$. Also, $(B \cap G) .(B \cap H) \theta^{-1}$ has precisely 3 abelian maximal subgroups.

(ii) If $(B \cap G) \cdot(B \cap H) \theta^{-1}=G$ then $B \cap G$ has a subgroup of scalars of index 4 , namely $\zeta(G)$.

Proof. Note that one of (i) or (ii) occurs. Write $B \cap G=B_{1}$ and $(B \cap H) \theta^{-1}=S$.

(i) Here, $Q=B_{1} \cap S$ is maximal in both $B_{1}$ and $S$. Since $Q \leq \zeta\left(B_{1} S\right) \leq B_{1} S$, we have that $\zeta\left(B_{1} S\right)=Q$. For otherwise, $B_{1} S / \zeta\left(B_{1} S\right)$ would be cyclic and $B_{1} S$ abelian, contradicting the irreducibility of $G$ by Lemma 2.3.1 (ii). Now $|B S: B|=2$, since $B S / B \cong B_{1} S / B \cap B_{1} S=B_{1} S / B_{1}$. Therefore,

$$
\left|B V_{\mathbf{4}}: B S\right|=|B G: B S|=|B G: B| /|B S: B|=2
$$

That is, $B S=B\langle t\rangle$ for some $t \in V_{4} \backslash\{1\}$. Since $Q$ is centralised by $B S$, we have $B\langle t\rangle \leq B C_{V_{4}}(Q)$. Now $Q$ is not $V_{4}$-trivial: if it were, then, since $\left|B_{1}: Q\right|=2$ and $B_{1} \geq F_{a} \leq C_{B}\left(V_{4}\right)$, we would have $B_{1}=Q \cdot F_{a}$. That is, $B_{1}$ would be a-trivial, contradicting faithfulness of this $V_{4}$-module. Thus $C_{V_{4}}(Q)=\langle t\rangle$.

An abelian maximal subgroup of $B_{1} S$ contains the centre $Q$ of $B_{1} S$; otherwise $B_{1} S$, as the product of two abelian subgroups that commute elementwise, would be abelian. Therefore, the abelian maximal subgroups of $B_{1} S$ are in one-to-one correspondence with the maximal subgroups of $B_{1} S / \zeta\left(B_{1} S\right) \cong C_{2} \times C_{2}$. 
(ii) If $G=B_{1} S$ then $B_{1} \cap S \leq \zeta(G)$. Certainly $\zeta(G) \leq B_{1}$ and $|G: \zeta(G)|>8$. For if we had $|G: \zeta(G)| \leq 4$ then $B_{1}$ would be either $\zeta(G)$ or $\zeta(G) . F_{a}$, and so would not be faithful. Hence

$$
16 \leq|G: \zeta(G)| \leq\left|G: B_{1} \cap S\right|=\left|B_{1}: B_{1} \cap S\right|\left|S: B_{1} \cap S\right|=16 .
$$

Thus $B_{1} \cap S=\zeta(G)$ is a $V_{4}$-trivial subgroup of $B_{1}$ of index 4 .

Remark 5.4.5 In Lemma 5.4.4 (ii), $G$ has class 2. A finite class 2 irreducible linear group of degree $n$ has central quotient of order $n^{2}$ in any case - see Isaacs (1976), Ex.2.13, p.31.

For ease of reference, in case (i) of Lemma 5.4.4 we say that $\theta$ is an isomorphism of type I; in case (ii), $\theta$ is an isomorphism of type II. A linear isomorphism between groups in the list of Theorem 5.4.1 which have more than one SCN4 subgroup is one of these types. We treat the linear isomorphisms of type I first. In this direction, we establish the following criterion, which is useful in testing for the existence of a nondiagonal SCN4 subgroup.

Lemma 5.4.6 Let $G$ be a finite irreducible subgroup of $B V_{4}$. Then $G$ has an SCN4 subgroup $S \neq B \cap G$ if and only if $B \cap G$ has a $V_{4}$-submodule $Q$ of index 2 such that $\left|C_{V_{4}}(Q)\right|=2$ and $\Phi(G) \leq Q$.

Proof. Suppose that $S$ exists. Since $G / B_{1}$ and $G / S$ are both isomorphic to $V_{4}$, we have $\Phi(G) \leq B_{1} \cap S$. Now we use Lemma 5.4.4: in case (i), this certainly gives the result. In case (ii), $G$ has class 2 and hence $B_{1}$ is noncyclic [by Berger, Kovács \& Newman (1980)], so that $B_{1} \geq F_{t}$ for some $t$ and $Q=\left(B_{1} \cap S\right) . F_{t}$ will do. Conversely, suppose that $Q$ exists, and denote by $t$ the involution of $V_{4}$ centralising $Q$. Any element of $t B \cap G$ has $t$-trivial square, which is therefore contained in $Q$, since $B \cap G$ is faithful. The subgroup $S$ generated by $Q$ and an element of $t B \cap G$ is abelian. Also, $\Phi(G) \leq Q<S$, so that $G / Q$ is elementary abelian and therefore $G / S \cong V_{4}$.

The next result is crucial. It shows that any two of the groups listed in Theorem 5.4.1 which are linearly isomorphic by a linear isomorphism of type I have diagonal subgroups with a common maximal subgroup.

Lemma 5.4.7 Let $G$ and $H$ be finite irreducible subgroups of $B V_{4}$, suppose that $G$ is mapped isomorphically onto $H$ by an isomorphism $\theta$ of type $\mathrm{I}$, and that $C_{V_{4}}(Q)=C_{V_{4}}(Q \theta)$ for $Q=(B \cap G) \cap(B \cap H) \theta^{-1}$. Then $Q \theta=Q$. 
Proof. We know from Lemma 5.4 .4 (i) that both $Q$ and $Q \theta$ are centralised by a single involution of $V_{4}$, say $t$, and that $B .(B \cap H) \theta^{-1}=B\langle t\rangle$. Choose an involution $s \neq t$ of $V_{4}$ and $s w \in s B \cap G$ : then $(s w) \theta \notin B \cap H$. If we had $(s w) \theta \in t B$, then we would have $\left(q^{s}\right) \theta=(q \theta)^{(s w) \theta}=q \theta$ for all $q \in Q$, since $t$ centralises $Q \theta$. This would imply that $Q$ is $V_{4}$-trivial, contradicting $C_{V_{4}}(Q)=\langle t\rangle$. Consequently, $(s w) \theta \notin t B$ and so $(s w) \theta \in s B\langle t\rangle$, whence it follows that the conjugation action of $s$ on $Q$ commutes with the action of $\theta$ on $Q$. Certainly $t$ commutes with $\left.\theta\right|_{Q}$, so that $\left.\theta\right|_{Q}$ is a $T$-module isomorphism of $Q$ onto $Q \theta$. By Proposition 2.1.3, it follows that $Q=Q \theta$.

The following lemma is easily proved by matrix multiplication.

Lemma 5.4.8 Let $Y_{1}$ be a subgroup of $Y, Y_{1}>B_{0}$. Then $N_{G L(4)}\left(Y_{1}\right)=N_{G L(4)}(Y)$ is the subgroup of $G L(4)$ generated by $b$ and all block diagonal matrices of the following form:

$$
f=\left(\begin{array}{cc}
f_{1} & 0 \\
0 & f_{2}
\end{array}\right)
$$

where $f_{1}, f_{2} \in G L(2)$.

Remark 5.4.9 Note that

$$
b f=\left(\begin{array}{cc}
0 & f_{2} \\
f_{1} & 0
\end{array}\right),
$$

and $C_{G L(4)}(Y)$ is precisely the set of all matrices of the form (5.21). Also, $C_{G L(4)}(Y)$ is normalised by $B V_{4}$ (but not by $B S_{4}$ ).

In the next two results we consider the following situation: $e \in G L(4)$ induces a linear isomorphism of type I between groups $G$ and $H$ in the list of Theorem 5.4.1. By Lemma 5.4.4 (i), $(B \cap G) \cap(B \cap H)^{e^{-1}}$ is a $V_{4}$-submodule of $B \cap G$ of index 2 and $C_{V_{4}}\left((B \cap G) \cap(B \cap H)^{e^{-1}}\right)=\langle t\rangle, C_{V_{4}}\left((B \cap G)^{e} \cap(B \cap H)\right)=\langle s\rangle$ for some involutions $t$ and $s$ of $V_{4}$.

Proposition 5.4.10 Let e, $G, H, t, s$ be as above. Then e may be written as rwfm or rwbfm, for some $r \in S_{3}, w \in U V$ and $m \in M(4)$, where $f$ is of the form (5.21) with

$$
f_{1}=f_{2}=\left(\begin{array}{rr}
1 & -1 \\
1 & 1
\end{array}\right)
$$


Proof. First, choose $r, r^{\prime} \in S_{3}$ such that $t^{r}=a=s^{r^{\prime}}$. Then note that $G^{r}$ and $H^{r^{\prime}}$ are finite irreducible subgroups of $B V_{4}$ such that $\left(G^{r}\right)^{r^{-1} e r^{\prime}}=H^{r^{\prime}}$. Furthermore, $r^{-1} e r^{\prime}$ induces a linear isomorphism of type I from $G^{r}$ onto $H^{r^{\prime}}$, and setting

$$
Q=\left(B \cap G^{r}\right) \cap\left(B \cap H^{r^{\prime}}\right)^{r^{-1}} e^{-1} r
$$

we see that

$$
C_{V_{4}}(Q)=C_{V_{4}}\left(Q^{r^{-1} e r^{\prime}}\right)=\langle a\rangle .
$$

Therefore, $Q \leq X Y$ but $Q \leq X$, and by Lemma 5.4.7, $Q^{r^{-1} e r^{\prime}}=Q$. We claim that $r^{-1} e r^{\prime}$ normalises $\left\langle y_{1}\right\rangle$. To see this, consider first the case of noncyclic $Q$. Then $\Omega_{1}(Q)=\Omega_{1}(X Y)$; since $r^{-1} e r^{\prime}$ normalises $\Omega_{1}(Q)$, it either fixes or interchanges the two nonscalar elements $x_{1} y_{1}^{ \pm 1}$ of this subgroup. In fact, since $x_{1}$ is a scalar, $r^{-1} e r^{\prime}$ either fixes or interchanges $y_{1}$ and $y_{1}^{-1}$, and the claim is true. Suppose next that $Q$ is cyclic. Then $r^{-1} e r^{\prime}$ normalises every subgroup of $Q$. Since $X Y \geq Q \mathbb{X}$ and $X\left\langle y_{1}\right\rangle$ is the unique minimal subgroup of $X Y / X$, there is a subgroup of the form $\left\langle x y_{1}\right\rangle(x \in X)$ in $Q$. Consequently, $y_{1}^{r^{-1} \text { er }}=x^{\prime} y_{1}$ for some $x^{\prime} \in X$. Now $\left|y_{1}\right|=\left|x^{\prime} y_{1}\right|=4 ;$ this implies that $x^{\prime} \in\left\langle x_{1}\right\rangle$ and hence $x^{\prime} \in B_{0} \leq\left\langle y_{1}\right\rangle$. Thus, $r^{-1}$ er again normalises $\left\langle y_{1}\right\rangle$, and this completes verification of the claim. By Lemma 5.4.8, $r^{-1} e r^{\prime} \in N_{G L(4)}(Y)$.

We know that $Q$ has index 2 in the abelian subgroup $\left(B \cap H^{r^{\prime}}\right)^{r^{\prime-1} e^{-1} r}$ of $G^{r}$, and that $C_{B V_{4}}(Q)=B\langle a\rangle$. Hence $\left(B \cap H^{r^{\prime}}\right)^{r^{\prime-1} e^{-1} r}=\langle a z, Q\rangle$ for some $a z \in G^{r}, z \in B$. Write $z=z_{1} z_{2}$ for some $z_{1} \in X Y$ and $z_{2} \in U V$. Now $r^{-1} e r^{\prime} \in N_{G L(4)}(X Y)$ and so $(a z)^{r^{-1} e r^{\prime}} \in B$ implies that $\left(a z_{2}\right)^{r^{-1} e r^{\prime}} \in B$. Choose $w \in U V$ such that $w^{2}=z_{2}$; then $a^{w r^{-1} e r^{\prime}}=\left(a z_{2}\right)^{r^{-1} e r^{\prime}} \in B$. Also, of course, wr $e r^{-1} \in N_{G L(4)}(Y)$. Therefore, $w r^{-1} e r^{\prime}$ is either $f$ or $b f$, for some $f$ of the form (5.21).

Note that $a$ itself is of the form (5.21), with blocks (12) [the matrix obtained by interchanging the rows of the $2 \times 2$ identity matrix]. By the choice of $f, a^{f}=a^{b f}$ is a diagonal matrix with nonzero entries \pm 1 , and so we see that $(12)^{f_{i}}$ is one of the diagonal matrices $(1,-1)$ or $(-1,1)$. Thus, the $f_{i}$ are of the form

$$
\left(\begin{array}{rr}
\mp \mu & \pm \nu \\
\mu & \nu
\end{array}\right)=\left(\begin{array}{rr}
\mp 1 & \pm 1 \\
1 & 1
\end{array}\right)\left(\begin{array}{ll}
\mu & 0 \\
0 & \nu
\end{array}\right) .
$$

Postmultiplication of an element of $G L(2)$ by (12) interchanges its columns. Therefore, up to postmultiplication by an element of $M(4), f$ has the stated form. The conclusion follows. 
Theorem 5.4.11 A group in the list of Theorem 5.4.1 with diagonal subgroup $F(i, j, 0,0,0,1, \xi)$, for some $i, j \geq 1$ and $\xi \in\{-1,0\}$, is $G L(4)$-conjugate by a linear isomorphism of type I to a group in the list with diagonal subgroup $F(i, j, 1,1,0,0)$ or $F(i, j, 0,0,0,1,1)$. The only other linear isomorphisms of type I between groups in the list are:

$$
\begin{aligned}
& \langle a, b, F(i, j, 0,0,0,1,1)\rangle \sim\left\langle a x_{i+1}, b, F(i, j, 1,1,0,0)\right\rangle \\
& \langle a, b, F(i, j, 0,0,1,1,1)\rangle \sim\left\langle a x_{i+1}, b, F(i, j, 1,1,1,0)\right\rangle, i, j \geq 1 \\
& \langle a, b, F(0,0,0,0,1,1,1)\rangle \sim\langle a, b, F(0,0,1,1,1,0)\rangle .
\end{aligned}
$$

Proof. Let $G, H, e, t$ and $s$ be as in the preamble before Proposition 5.4.10. We repeat some of the argument in the proof of that proposition, distinguishing two cases: $t=a$ and $t \neq a$. First, we make some preliminary observations. For $f$ as in Proposition 5.4.10,

$$
\begin{aligned}
& a^{f}=x_{1} u_{1}^{-1}, \\
& b^{f}=b, \\
& u_{1}^{f}=a x_{1}^{-1} .
\end{aligned}
$$

In Theorem 5.4.1, each $G$ is written in the form $G=\left\langle a x_{a} y, b x_{b} u v, B \cap G\right\rangle$. In these terms, if we set $Q=\left(B \cap G^{r}\right) \cap(B \cap H)^{e^{-1} r}$, then the following conditions are equivalent to $\Phi(G) \leq Q^{r^{-1}}$ :

$$
\begin{aligned}
& x_{a}^{2} y^{2} \in Q^{r^{-1}}, \\
& x_{b}^{2} u^{2} \in Q^{r^{-1}}, \\
& y^{2} u^{2} v^{-2} \in Q^{r^{-1}} .
\end{aligned}
$$

Case 1: $t=a$. Here, $r=1$ and $Q=(B \cap G) \cap(B \cap H)^{e^{-1}}$. Now $C_{B}(\langle a\rangle)=X Y$ and so $Q \leq(B \cap G) \cap X Y$. By maximality of $Q$ and faithfulness of $B \cap G$, this implies that $Q=(B \cap G) \cap X Y$. Observe then that (5.25) is automatically satisfied in this case. Also, since $(B \cap G)(B \cap H)^{e^{-1}} \leq B\langle a\rangle \cap G$, it follows that $(B \cap G)(B \cap H)^{e^{-1}}=\left\langle a x_{a} y, B \cap G\right\rangle$.

Obviously $Q$ is noncyclic: otherwise, $B \cap G$ would be $Q \cdot F_{a}$ and hence would not be faithful. In Example 5.2.7 we determined the finite noncyclic $V_{4}$-submodules of $B$ which are centralised by $\langle a\rangle$, and, with reference to Figures 5.1 and 5.2 , the $V_{4}$-submodules which contain them as maximals. By considering only the faithful ones among the latter, we see that either $Q=F(i, j, 0,0,0,1)$ and $B \cap G$ is 
$F(i, j, 1,1,0,0)$ or one of the $F(i, j, 0,0,0,1, \xi)$; or $Q=F(i, j, 0,0,1,1)$ and $B \cap G$ is one of $F(i, j, 0,0,1,1,1), F(i, j, 1,1,1,0)$. Case by case inspection, with reference to (5.2), (5.4) and (5.6), shows that $u_{1} \in(B \cap G) X Y$. So choose $g \in G \cap X Y u_{1}$; then

$$
\begin{gathered}
\left\langle a x_{a} y, Q\right\rangle, \\
\left\langle a x_{a} y g, Q\right\rangle, \\
B \cap G
\end{gathered}
$$

are three distinct abelian maximal subgroups of $\left\langle a x_{a} y, B \cap G\right\rangle=(B \cap G)(B \cap H)^{e^{-1}}$ Thus, by the second claim of Lemma 5.4.4 (i), $(B \cap H)^{e^{-1}}$ must be one of $\left\langle a x_{a} y, Q\right\rangle$ or $\left\langle a x_{a} y g, Q\right\rangle$. Recall the way $w \in U V$ was chosen in the proof of Proposition 5.4.10: here, $z_{2}=1$ or $z_{2}=u_{1}$ and, therefore, we may take $w=u_{2}^{\tau}$ with $\tau \in\{0,1\}$ accordingly. By Proposition 5.4.10 we conclude that $e=b^{\sigma} u_{2}^{\tau} f m$, where $\sigma, \tau \in\{0,1\}, m$ is a monomial matrix, and $f$ is as stated in that proposition.

In this case $(t=a)$, all linear isomorphisms of type I between groups in the list of Theorem 5.4.1 may be identified by the following procedure. For each $G$ in the list, check whether $B \cap G$ is one of the options identified above, then determine whether $G$ has an SCN4 subgroup different from $B \cap G$; that is, by Lemma 5.4.6, determine whether (5.26) and (5.27) are satisfied for the corresponding $Q$. For each such $G$ satisfying these conditions, calculate the (four) conjugates $G^{b^{\sigma}} u_{2}^{r} f$. We will see that these conjugates are always in $B S_{4}$ and, of course, they contain $F_{a}$. Then determine the (unique) $B$-conjugate of each of these groups in the list of Theorem 5.4.1. Thus, by Remark 5.4.3, all possible linear isomorphisms of type I in this case are accounted for by this procedure. We will follow this procedure in general; however, as in the next paragraph, it is not always necessary to consider all four conjugates as indicated. For the moment we will assume $|Q|>4$, so that $\left\langle x_{1}, y_{1}\right\rangle \leq Q$.

Suppose that $Q=F(i, j, 0,0,0,1)$. When $B \cap G=F(i, j, 0,0,0,1, \xi)$ for $\xi \neq 1$, we may verify by (5.26), (5.27) and inspection of the list of Theorem 5.4 .1 that $\Phi(G) \leq Q$. Using (5.22)-(5.24), we may also check that $G^{f} \leq B S_{4}$ and $B \cap G^{f}$ is one of the following (remember that we assume $x_{1} \in Q$ ):

$$
\begin{aligned}
\left\langle a x_{i+1}^{\varepsilon}, x_{i}, y_{j}\right\rangle^{f} & =\left\langle x_{i+1}^{\varepsilon} u_{1}, x_{i}, y_{j}\right\rangle \\
& = \begin{cases}F(i, j, 1,1,0,0) & \varepsilon=0 \\
F(i, j, 0,0,0,1,1) & \varepsilon=1\end{cases}
\end{aligned}
$$

By Theorem 5.4.1 and Remark 5.4.2, $G^{f}$ is $B S_{4}$-conjugate to one of the groups in the list of Theorem 5.4.1, with either $F(i, j, 1,1,0,0)$ or $F(i, j, 0,0,0,1,1)$ as diagonal 
subgroup, and so we have proved the first statement of the theorem. If $\xi=1$ then by $(5.26),(5.27)$ and Theorem 5.4.1, we see that $G$ is one of the $\left\langle a y_{j+1}^{\eta}, b, B \cap G\right\rangle$. Each of these groups is obviously normalised by $b$, and therefore it is enough to calculate the two conjugates $G^{u_{2}^{\top} f}$. If $\eta=1$ then by $(5.22)-(5.24)$,

$$
\begin{aligned}
G^{u_{2}^{\tau} f} & =\left\langle a y_{j+1} u_{1}^{\tau}, b, x_{i+1} u_{1}, x_{i}, y_{j}\right\rangle^{f} \\
& =\left\langle a^{\tau} y_{j+1} u_{1}, b, a x_{i+1}, x_{i}, y_{j}\right\rangle \\
& = \begin{cases}\left\langle a x_{i+1}, b, F(i, j, 0,0,0,1,0)\right. & \tau=0 \\
\left\langle a x_{i+1}, b, F(i, j, 0,0,0,1,-1)\right. & \tau=1\end{cases}
\end{aligned}
$$

remember that we assume $x_{1} \in Q$. These linear isomorphisms have already been noted. If $\eta=0$ then by $(5.22)-(5.23)$ we have

$$
\begin{aligned}
G^{u_{2}^{\tau} f} & =\left\langle a^{\tau} u_{1}, b, a x_{i+1}, x_{i}, y_{j}\right\rangle \\
& \sim_{B} \begin{cases}\left\langle a x_{i+1}, b, F(i, j, 1,1,0,0)\right. & \tau=0 \\
G & \tau=1\end{cases}
\end{aligned}
$$

For $\tau=0$, this gives the first of the linear isomorphisms listed explicitly in the statement of the theorem. When $B \cap G=F(i, j, 1,1,0,0)$, we see from Theorem 5.4.1 that $G$ is still normalised by $b$. By (5.26) and (5.27), $G$ is one of the $\left\langle a x_{i+1}^{\varepsilon} y_{j+1}^{\eta}, b x_{i+1}^{\gamma(1-\varepsilon)}, B \cap G\right\rangle$. By $(5.22)-(5.24)$,

$$
G^{u_{2}^{r} f} \sim_{B}\left\langle a, b x_{i+1}^{\gamma(1-\varepsilon)}, x_{i+1}^{\varepsilon} y_{j+1}^{\eta} u_{1}, x_{i}, y_{j}\right\rangle
$$

In fact, $G^{u_{2}^{\top} f} \sim_{B} G$ or $u_{2}^{\tau} f$ induces a linear isomorphism already noted.

Now suppose that $Q=F(i, j, 0,0,1,1)$. When $B \cap G=F(i, j, 0,0,1,1,1)$, it is clear by (5.26), (5.27) and Theorem 5.4.1 that $G$ must be the relevant split extension. This is certainly normalised by $b$, and we have

$$
G^{u_{2}^{r} f} \sim_{B} \begin{cases}\left\langle a x_{i+1}, b, F(i, j, 1,1,1,0)\right. & \tau=0 \\ G & \tau=1 .\end{cases}
$$

For $\tau=0$, this gives the second of the explicitly listed conjugacies. When $B \cap G=F(i, j, 1,1,1,0), G$ is one of the $\left\langle a x_{i+1}^{e}, b, B \cap G\right\rangle$. These groups are normalised by $b$ and, as in the previous paragraph, it may be checked that either $G^{u_{2}^{\tau} f} \sim_{B} G$ or $u_{2}^{\tau} f$ induces a linear isomorphism which has already been noted.

Now we consider the case that $|Q|=4$. Explicitly, $Q=F_{a}=F(0,0,0,0,1,1)$. From Figure 5.2, we see that $B \cap G=F(0,0,0,0,1,1,1)$ or $B \cap G=F(0,0,1,1,1,0)$. 
Then the usual reasoning shows that $G$ must be either $\langle a, b, F(0,0,0,0,1,1)\rangle$ or one of the $\left\langle a x_{1}^{\varepsilon}, b, F(0,0,1,1,1,0)\right\rangle$. These groups are normalised by $b$, and conjugation by the $u_{2}^{\tau} f$ yields the last of the explicitly listed linear isomorphisms, and no new ones.

Case 2: $t \neq a$. (By symmetry, of course, we may also assume that $s \neq a$.) In this case, $Q=\left(B \cap G^{r}\right) \cap(B \cap H)^{e^{-1} r}$, where $r=(13)$ or $r=(23)$; also, (5.25) is not necessarily satisfied for all $G$. We claim that $Q$ may be assumed to be cyclic. To see this, suppose that $Q$ is noncyclic; that is, $(B \cap G) \cap(B \cap H)^{e^{-1}} \geq F_{t}$. Then $B \cap G^{r}$ contains one of $F(i, j, 0,0,0,1)$ or $F(i, j, 1,1,1,0)$ as maximal. Thus, as we saw above, $B \cap G^{r}$ certainly occurs as the diagonal subgroup of a group in the list of Theorem 5.4.1. Since by hypothesis $G$ is in that list, we have by Remark 5.4 .2 that $(B \cap G)^{r}=B \cap G$. In particular, $B \cap G$ has a maximal $V_{4}$-submodule with centraliser $\langle a\rangle$ in $B$. The faithful finite $V_{4}$-submodules of $B$ with such a maximal submodule were listed in Case 1; of these, we see from the orbit list given in the proof of Proposition 5.2.8 that only the $F(i, 1,1,1,0,0)$ and $F(0,0,0,0,1,1,1)$ are normalised by $r$. For $r=(13)$ and $r=(23)$ in turn, we follow the procedure established in Case 1 (here, $w$ may still be chosen as $u_{2}^{\tau}$ ) for each $G$ satisfying (5.25)(5.27) and with one of the aforementioned $V_{4}$-modules as diagonal subgroup. This will yield all linear isomorphisms of type I between such $G$ and other groups in the list of Theorem 5.4.1. Details of these calculations are omitted: no new isomorphisms are obtained, and so from now on we assume that $Q$ is cyclic.

For given cyclic $Q$, there is a single possibility for $B \cap G: B \cap G=F_{a} Q^{r^{-1}}$. Similarly, $B \cap H=F_{a} Q^{r^{\prime-1}}$. Since these two $V_{4}$-submodules of $B$ are conjugate by (12), it follows from Remark 5.4.2 that $B \cap G=B \cap H$ and we may assume that $t=s, r=r^{\prime}$. Thus, to determine the possibilities for $B \cap G$, we begin by listing the finite cyclic $V_{4}$-submodules of $B$ with centraliser $\langle a b\rangle$, say, in $B$. This listing is carried out using the same method as in the proof of Lemma 5.1.1; again, the details are omitted. We obtain the following list:

$$
\begin{gathered}
\left\langle v_{l}\right\rangle, \\
\left\langle x_{1} v_{l+1}\right\rangle, \\
\left\langle x_{l+1} v_{1}\right\rangle,
\end{gathered}
$$

where $l \geq 1$. For each submodule $M$ in this list, only one of $F_{a} M$ or $F_{a} M^{(12)}$ occurs in the list of Theorem 5.4.1. We select this as a possibility for $B \cap G=B \cap H$. It is 
straightforward to verify that $B \cap G=B \cap H$ must be one of the $F(0,0,1, l, 1,0)$, $F(0,0, l, 1,1,0,1)$ or $F(l, 1,0,0,0,1,1)$. In the first situation, by $(5.25)-(5.27)$ and inspection of the list of Theorem 5.4.1, we see that $G$ is one of the two groups $\left\langle a x_{1}^{\varepsilon}, b, B \cap G\right\rangle$. In fact, these groups appear as $D^{+}\left(2^{l+4}\right)$ and $Q^{+}\left(2^{l+4}\right)$ in the proof of Proposition 5.2.1. Berger, Kovács \& Newman (1980) show that these groups are nonisomorphic and so there is nothing to prove here (remember that $B \cap G=B \cap H$ ). In either of the other situations, inspection shows that only the splitting extension satisfies (5.25)-(5.27). Again, there is nothing to prove, and so we are done.

Remark 5.4.12 A group of order 32 with a self-centralising normal subgroup of index 4 and centre of order 2 has a faithful irreducible representation of degree 4 . By Marshall Hall \& Senior (1964), there are seven such nonisomorphic groups. There are eight groups in the list of Theorem 5.4 .1 of order 32 ; using Theorem 5.4 .11 , we omit one of these. The remaining groups are therefore nonisomorphic.

To complete step 3 as outlined in the discussion at the beginning of this section, all that remains is to determine the linear isomorphisms of type II. As we know (see Remark 5.4.5), a group in the list of Theorem 5.4.1 involved in an isomorphism of this type has class 2 . In the next lemma, we give some useful facts about these groups. Recall that the centre of an irreducible linear group (of finite degree) consists of scalars.

Lemma 5.4.13 Let $G$ be a finite irreducible subgroup of $B V_{4}$, and suppose that $G$ has class 2 . Then

(i) $[B \cap G, G]=B_{0}$.

Furthermore, if $G$ splits over $B \cap G$ then

(ii) $\exp (G)=\max \{\exp (B \cap G), 4\}$.

Proof. (i) Certainly $[B \cap G, G] \leq[G, G] \leq X$. Also, $G^{2} \leq B \cap G$ and consequently, for all $z \in B \cap G$ and $g \in G$, we have $1=\left[z, g^{2}\right]=[z, g][z, g]^{g}=[z, g]^{2}$. Hence, $[B \cap G, G] \leq \Omega_{1}(X)=B_{0}$.

(ii) By replacing $G$ with the appropriate $B V_{4}$-conjugate if necessary, we may assume by Lemma 5.3.10 that $V_{4}<G$. For all $t \in V_{4}, z \in B \cap G$ and $n \geq 1$,

$$
(t z)^{n}=t^{n} z^{n}[z, t]^{\left(\begin{array}{l}
n \\
2
\end{array}\right)} .
$$


If $|z| \geq 4$ then 2 divides $\left(\begin{array}{l}n \\ 2\end{array}\right)$ and by (5.28) and (i), $(t z)^{|z|}=1$. If $|z| \leq 2$ then $|t z| \leq 4$, again by (5.28) and (i). In either case, $G$ has an element of order 4 . For if not, we would have $(t z)^{2}=1$ and then $z^{t}=z$ for all $t \in V_{4}$ and $z \in B \cap G$, contradicting the faithfulness of $B \cap G$. Certainly $B \cap G$ has an element of order $\exp (B \cap G)$, and so the conclusion follows.

Next, we show that any linear isomorphisms of type II between groups in the list of Theorem 5.4.1 have already been determined in Theorem 5.4.11.

Proposition 5.4.14 If two groups in the list of Theorem 5.4.1 are conjugate by a linear isomorphism of type II, then they are conjugate by a linear isomorphism of type I.

Proof. Let $G$ be a group as in the statement of the proposition. By Lemma 5.4.4 (ii), $|B \cap G: \zeta(G)|=4$ and by Remark 5.4 .12 , we may assume that $|\zeta(G)| \geq 4$.

By the definition of type II isomorphism, $G$ has an SCN4 subgroup, different from $B \cap G$, which supplements $B$ in $B V_{4}$. This implies that there are elements $a z$ and $b w$ of $G, z, w \in B$, such that $[a z, b w]=1$. Since $G$ has class 2 , we have the following commutator identity, true for all elements $g_{1}, \ldots, g_{4}$ of $G$ :

$$
\left[g_{1} g_{2}, g_{3} g_{4}\right]=\left[g_{1}, g_{3}\right]\left[g_{1}, g_{4}\right]\left[g_{2}, g_{3}\right]\left[g_{2}, g_{4}\right]
$$

Hence, by Lemma 5.4 .13 (i), $[a z, b w] \in B_{0}$ for all $a z, b w \in G$. Since $G$ is in the list of Theorem 5.4.1, it may be written as $G=\left\langle a x_{a} y, b x_{b} u v, B \cap G\right\rangle$, so that $[a y, b u v] \in B_{0}$. Consequently, $y^{-2} u^{2} v^{2} \in B_{0}$ and moreover,

$$
y^{2}, u^{2}, v^{2} \in B_{0}
$$

By assumption, $\zeta(G)=\left\langle x_{i}\right\rangle$ for some $i \geq 1$. Then $\zeta(G) \cdot F_{a}=F(i, 1,0,0,0,1)$ is a maximal subgroup of $B \cap G$. As we saw in the proof of Theorem 5.4.11, this means that $B \cap G$ is $F(i, 1,1,1,0,0)$ or one of the $F(i, 1,0,0,0,1, \xi)$. For the purposes of this proposition, and by Theorem 5.4.11, it clearly suffices to consider $B \cap G=F(i, 1,1,1,0,0)$ or $B \cap G=F(i, 1,0,0,0,1,1)$. By (5.29) and inspection of the list in Theorem 5.4.1, it may be verified that $G$ must be one of the following groups:

$$
\begin{gathered}
\langle a, b, F(i, 1,1,1,0,0)\rangle, \\
\left\langle a x_{i+1}, b, F(i, 1,1,1,0,0)\right\rangle, \\
\langle a, b, F(i, 1,0,0,0,1,1)\rangle .
\end{gathered}
$$


By Theorem 5.4.11, the second and third of these groups are isomorphic. However, they are not isomorphic to the first: by Lemma 5.4 .13 , the first group has exponent $2^{i+1}$, whereas the second group contains the element $a x_{i+1}$ of order $2^{i+2}$.

By Proposition 5.4.14 and Theorems 5.4.1 and 5.4.11, we have the following summary of our solution to the listing problem in the case $T=V_{4}$.

Theorem 5.4.15 Modify the list of Theorem 5.4.1 by omitting

- the $\langle a, b, F(i, j, 0,0,1,1,1)\rangle$,

- the $\langle a, b, F(i, j, 0,0,0,1,1)\rangle$,

- all groups with diagonal subgroup $F(i, j, 0,0,0,1, \xi), \xi \neq 1$.

Then this modified list is a complete and irredundant list of GL(4)-conjugacy class representatives of finite irreducible subgroups of $B V_{4}$.

By Theorem 2.4.1, no two groups in the list of Theorem 5.4.15 with unique SCN4 subgroups that are different are even isomorphic. However, it is not known whether this conclusion extends to groups with more than one SCN4 subgroup. 


\section{References}

Frank W. Anderson and Kent R. Fuller (1973), Rings and Categories of Modules, Graduate Texts in Math., 13. Springer-Verlag Inc., New York.

J. H. Benson and J. H. Conway (1985), "Diagrams for modular lattices", J. Pure Appl. Algebra, 37, 111-116.

T.R. Berger and L.G. Kovács and M.F. Newman (1980), "Groups of prime power order with cyclic Frattini subgroup", Nederl. Akad. Wetensch. Proc. Ser. A, 83(1), 13-18.

G. Birkhoff (1935), "On the structure of abstract algebras", Proc. Camb. Phil. Soc., 30, 433-454.

Kenneth S. Brown (1982), Cohomology of Groups, Graduate Texts in Math., 87. Springer-Verlag, New York, Heidelberg, Berlin.

S.B. Conlon (1976), " $p$-groups with an abelian maximal subgroup and cyclic center", J. Austral. Math. Soc. Ser. A, 22, 221-233.

C.W. Curtis and I. Reiner (1962), Representation Theory of Finite Groups and Associative Algebras (2nd edition), Pure and Appl. Math., 11. Interscience, New York.

Charles W. Curtis and Irving Reiner (1981), Methods of Representation Theory (with Applications to Finite Groups and Orders), Volume 1. John Wiley \& Sons, New York, Chichester, Brisbane, Toronto.

John D. Dixon (1971), The structure of linear groups. Van Nostrand Reinhold, London. 
Larry Dornhoff (1971), Group representation theory, Part A. Marcel Dekker, New York.

George Grätzer (1971), Lattice theory. W.H. Freeman and Company, San Francisco.

Karl W. Gruenberg (1970), Cohomological Topics in Group Theory, Lecture Notes in Math., 143. Springer-Verlag, Berlin, Heidelberg, New York.

P.J. Hilton and U. Stammbach (1971), A Course in Homological Algebra, Graduate Texts in Math., 4. Springer-Verlag, New York, Heidelberg, Berlin.

J. Huebschmann (1980), "Crossed $n$-fold extensions of groups and cohomology", Comm. Math. Helv., 55, 302-313.

Johannes Huebschmann (1981), "Automorphisms of Group Extensions and Differentials in the Lyndon-Hochschild-Serre spectral sequence", J. Algebra, 72(2), $296-334$.

I.M. Isaacs (1976), Character Theory of Finite Groups, Pure and Applied Mathematics, 69. Academic Press, Berlin, Heidelberg, New York.

Saunders Mac Lane (1963), Homology. Springer-Verlag, Berlin, Göttingen, Heidelberg.

Marshall Hall, Jr. and James K. Senior (1964), The Groups of Order $2^{n}(n \leq 6)$. Macmillan, New York.

P.M. Neumann (1964), "On the structure of standard wreath products of groups", Math. Z., 84, 343-373.

Robert Remak (1930), "Über die Darstellung der endlichen Gruppen als Untergruppen direkter Produkte", J. Reine Angew. Math., 163, 1-44.

Derek J. Robinson (1981), "Applications of cohomology to the theory of groups", C.M. Campbell and E.F. Robertson (Eds.), Group-St Andrews 1981, LMS Lecture Notes, 71 , pp. $46-80$.

Derek J. Robinson (1982), A Course in the Theory of Groups, Graduate Texts in Math., 80. Springer-Verlag, New York, Heidelberg, Berlin. 


\title{
Index
}

\author{
$B, 5$ \\ $C, 5$ \\ $D, 5$ \\ $D(4), 2$ \\ $E_{\tau}^{p, q}, 26,27$ \\ $F_{t}, 66$ \\ $F_{t}^{+}, F_{t}^{-}, 67$ \\ $F\left(i, j, k, l, \delta_{1}, \delta_{2}\right), 72$ \\ $F\left(i, j, k, l, 0,0, \xi_{1}, \xi_{2}\right), 73$ \\ $F(i, j, k, l, 0,1, \xi), 73$ \\ $F(i, j, k, l, 1,0, \xi), 73$ \\ $F(i, j, k, l, 1,1,1), 73$ \\ $F\left(i, j, k, l, 0,0,2, \alpha_{1}, \alpha_{2}\right), 74$ \\ $F(i, j, k, l, \delta, \xi, \alpha), 74$ \\ $G L(n), 1$ \\ $G L(n, p), 2$ \\ $M(4), 2$ \\ $S_{4}, 2$ \\ $U^{\prime} f^{-1}, 35$ \\ $U^{G}, 17$ \\ $U^{\perp}, 9$ \\ $V(n, q), 5$ \\ $V_{4}, 5$ \\ $X, Y, U, V, 70$ \\ $a, b, c, 66$ \\ $d_{r}^{p, q}, 26,27$ \\ $\Omega_{k}(G), 5$ \\ $\Phi(G), 4$
}

$\zeta(G), 4$

$\alpha_{i}, 11$

$[\alpha], 48$

¿, 71

$\operatorname{Aut}(N, U), 31$

$\operatorname{Aut}_{G}(E), 32$

Coind $_{H}^{G} U, 24$

$\operatorname{Der}(G, U), 18$

$\operatorname{Hom}_{G}\left(U_{1}, U_{2}\right), 17$

$\operatorname{Ider}(G, U), 18$

$\operatorname{Ind}_{H}^{G} U, 24$

$\mathrm{Out}_{G}(E), 32$

$\operatorname{rk}(G), 5$

$\sim, 88$

$\sim_{S}, 88$

$\mathrm{F}_{q}, 5$

$\mathcal{L}(W), 45$

$\mathcal{L}(n, q), 64$

$\mathcal{L}(U) \times \mathcal{L}(V), 46$

annihilator, 8

atlas, 58

complete and irredundant, 59

augmentation map, 17

$n$-coboundary, 18

$n$-cochain, 17

$n$-cocycle, 18

compatibility, 23

$G$-complex, 16 
coupling, 20

derivation, 18 inner, 18

differentials

in a $G$-complex, 16

in a spectral sequence, 26

edge

Cartesian, 46

composition, 53

restriction, 52

edge homomorphism

on the base, 26

on the fibre, 26

equivalent extensions, 19

family, 72

Hasse diagram, 44

homomorphism

restriction, 28

inflation, 28

inducibility, 23

interval, 44

interval of definition, 49

maximal, 76

isomorphism

linear, 1

of type I, 97

of type II, 97

isomorphism question, 14

low section, 45

module

cocyclic, 67 coinduced, 24

cyclic, 67

faithful, 11

induced, 24

monogenic, 7

obstruction, 24

page, 58

perspectivity, 53

projection group, 3

$G$-projective resolution, 17

standard, 17

radical, 7

Remak's theorem, 48, 50, 57, 75

ring

local, 7

quasi-Frobenius, 7

socle, 8

spectral sequence

cohomology, 26

convergence of, 26

LHS, 27

subgraph, 45

subgroup

diagonal, 3

SCN4, 97

sublattice

Cartesian product, 46

convex, 44

submodule

Cartesian, 46, 50

diagonal, 50

subposet, 44 
transversal function, 19 